\title{
Brain Dicer1 is down-regulated in a mouse model of Alzheimer's disease via AB42-induced repression of nuclear factor erythroid 2-related factor 2
}

\section{Yan Wang}

Wenzhou Medical University School of Optometry and Ophthalmology

Meiling Lian

Wenzhou Medical University School of Optometry and Ophthalmology

Jing Zhou

Wenzhou Medical University School of Optometry and Ophthalmology

shengzhou wu ( $\square$ wszlab@mail.eye.ac.cn )

Wenzhou Medical University School of Optometry and Ophthalmology https://orcid.org/0000-00031154-2369

\section{Research article}

Keywords: Dicer1, Alzheimer's disease, hippocampus, APPswe/PSEN1dE9 mice, Nrf2, antioxidant response element囚oxidative stress, neurodegeneration, gene regulation, neurotoxicity

Posted Date: May 7th, 2020

DOl: https://doi.org/10.21203/rs.3.rs-25902/v1

License: @ (i) This work is licensed under a Creative Commons Attribution 4.0 International License.

Read Full License

Version of Record: A version of this preprint was published at Molecular Neurobiology on July 31st, 2020. See the published version at https://doi.org/10.1007/s12035-020-02036-8. 
Brain Dicer1 is down-regulated in a mouse model of Alzheimer's disease via A 342 -induced repression of nuclear factor erythroid 2-related factor 2

Yan Wang ${ }^{\text {a, b}}$, Meiling Lian ${ }^{\text {a,b }}$, Jing Zhou ${ }^{a, b}$, Shengzhou $W^{*}$ a,b

Yan Wang and Meiling Lian equally contributed to the work

${ }^{a}$ School of Optometry and Ophthalmology and the Eye Hospital, Wenzhou Medical

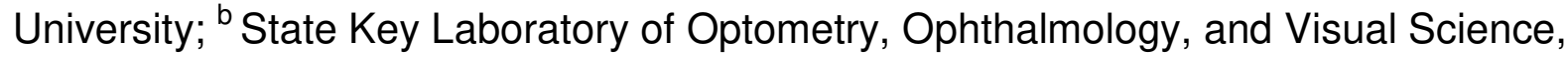
270 Xueyuan Road, Wenzhou, Zhejiang, 325003, P.R.China

Running title: Chronic $A \beta$ exposure reduces Dicer1 via Nrf2-ARE signaling

Correspondence should be directed to:

Prof. Shengzhou Wu, Ph.D, M.D

School of Optometry and Ophthalmology,

Wenzhou Medical University, Wenzhou, China

Email:wszlab@mail.eye.ac.cn or wszlab@163.com;

Phone:(86)-577-88067974;

Fax:(86)-577-88067934

Email addresses: Yan Wang, aiwangyan1987@sina.com; Meiling Lian, 13736931292@163.com; Jing Zhou, jingz454670925@163.com; Shengzhou Wu, wszlab@mail.eye.ac.cn 


\section{Abstract}

\section{Background}

Oxidative stress critically underlies the neurodegenerative pathogenesis of Alzheimer's disease (AD). Depletion of Dicer1, an endoribonuclease central to microRNA maturation, also leads to neurodegeneration. We therefore hypothesized that altered Dicer1 expression may play a role in $A D$.

\section{Results}

Using immunoblotting and quantitative real-time PCR, we found that Dicer1 protein and mRNA levels were reduced in the hippocampi of animals of the AD mouse model APPswe/PSEN1dE9 compared with littermate controls. SiRNA-meditated Dicer1 knockdown induced oxidative stress, reduced mitochondrial intermembrane potential, and increased apoptosis in cultured neurons. A 342 exposure decreased Dicer1 and also down-regulated the oxidative stress-induced transcriptional regulator nuclear factor erythroid 2-related factor 2 (Nrf2). Conversely, Nrf2 overexpression increased Dicer1 mRNA and protein levels and reverted the A $\beta 42$-induced Dicer1 reduction. To further investigate Dicer1 regulation, we cloned Dicer1 promoter variants harboring the Nrf2binding site, the antioxidant response elements (ARE), into a luciferase reporter and found that simultaneous transfection of Nrf2-expressing plasmid increased luciferase expression from these promoter constructs. ChIP assays indicated that Nrf2 directly interacted with the ARE motifs in the Dicer1 promoter. Furthermore, Dicer1 
overexpression in cultured neurons reverted $A \beta 42$-induced neurite deficits. Of note, injection of Dicer1-expressing adenovirus into the hippocampus of the AD mice significantly improved spatial learning.

\section{Conclusions}

These findings indicate that Dicer1 expression is reduced in the AD brain and that chronic $A \beta$ exposure decreases Dicer1 levels in neurons via Nrf2-ARE signaling. Our results uncover a significant role for Dicer1 in AD and highlight that Dicer1 expression responds to oxidative stress in the brain.

\section{Keywords}

Dicer1, Alzheimer's disease, hippocampus, APPswe/PSEN1dE9 mice, Nrf2, antioxidant response element, oxidative stress, neurodegeneration, gene regulation, neurotoxicity 


\section{Background}

Alzheimer's disease $(A D)$ is an inexorable neurodegenerative disorder, characterized with extracellular amyloid plaque deposition, intracellular neurofibrillary tangle formation, extensive neuronal loss, and cognitive dysfunction [1, 2]. The pathogenesis of $A D$ involves the key component of oxidative stress which is dependent on the balance between reactive species and anti-oxidation systems [3]. Accumulation of reactive oxygen/nitrogen species generates oxidative stress and produces ensued cytotoxicity [4] as well as does ferroptosis due to iron accumulation in AD brain $[5,6]$. Amyloid peptide inactivates membrane channel and transporter including sodium/calcium exchanger, calcium ATPase, glutamate and glucose transporter--these hazardous effects involve the roles of reactive oxygen species and lipid peroxidation[79]. Anti-oxidation systems are used to cope with oxidative injury, in which the regulation of the key enzymes or proteins, used for synthesizing glutathione or recycling peroxiredoxins, revolve around a transcriptional factor, nuclear factor erythroid 2related factor $2(\mathrm{Nrf2})[10,11]$. Nrf2 is a basic leucine zipper protein that regulates expression of a multitude of antioxidant proteins in response to oxidative injuries. Under quiescent situation, Nrf2 is retained in cytoplasm by Keap1, but upon oxidative stimulation, it is released from the binding and translocated into nucleus, triggering the transcription of antioxidant genes [12].

Human Dicer1 is an enzyme with multidomains consisting of an amino-terminal domain with helicase activity, a DUF383 domain with unknown function, a PAF (PiwiArgonaute-Zwille) domain, two RNase III domains, and a carboxyl-terminal domain for double-stranded RNA binding [13]. In addition to traditional roles such as in generating 
small regulatory RNAs including miRNAs or siRNAs [14], Dicer1 also participates in maintaining heterochromatin structure [15, 16], involving in inflammation [17, 18], and breaking chromosomal DNA in Caenorhabditis elegans undergoing apoptosis [19]. Degeneration of retinal pigment epithelial cell (RPE), a characteristic of advanced dry age-related macular degeneration (AMD), is associated with Dicer1 depletion through which AluRNA, a specific substrate of Dicer1 degradation, accumulates and results in inflammasome activation and MyD88 signaling which lead to ensued cell death [20, 21]. In the central nervous system, Dicer1 is widely expressed in neurons and glia. Conditional loss of Dicer1 in neuron or glia produces different phenotypes and differentially impacts the brain function--the inducible impairments depend on developmental stages [22-25]. RPE degeneration induced by Dicer1 depletion involves inflammasome activation which likely accompanies with oxidative stress which is an etiological factor in neurodegeneration occurring in $A D$. As such, we exploited the roles of Dicer1 in $A D$ and further explored whether Dicer1 expression was regulated by Nrf2, a transcription factor balancing redox level. We further investigated potential therapy by overexpressing Dicer1 in the hippocampus of APPswe/PSEN1dE9 (APP/PS1) mice. 


\section{Materials and methods}

\section{Materials}

The following primary antibodies were used in this study: Dicer1(Sigma, St Louis, MO, USA, cat\# WH0023405M1, research resource identifier, RRID: AB_1841286), caspase 3 (Santa Cruz Biotechnology, Santa Cruz, CA, USA, cat\# sc-271759, RRID: AB_10709891), Keap 1(Proteintech, Suzhou, China, cat\# 60027-1-Ig, RRID: AB_2132623), and rabbit polyclonal anti-Nrf2 (Proteintech, cat\# 16396-1-AP, RRID:AB_2782956). Activated caspase 3 (Beyotime Biotechnology, Haimen, China, cat\# AC033), ßIII-tubulin (Beyotime Biotechnology, cat\# AT809), Histone 3 (Beyotime Biotechnology, cat\# AF0009), Vimentin (Beyotime Biotechnology, cat\# AF1975), GAPDH (Bioworld, Nanjing, China, cat\# MB001), NeuN (Beyotime Biotechnology, cat\# AF1072). The following secondary antibodies were also used in this study: goat antimouse horseradish peroxidase conjugated IgG (Boster Biological Technology, Co. Ltd, Wuhan, China), goat anti-Rabbit horseradish peroxidase conjugated IgG (Boster Biological Technology). The following reagents or cell lines were used in this study: a human Aß42 peptide (GenScript, Nanjing, China, cat\# RP10017), the Dicer1 siRNA duplex and the negative control (NC) siRNA duplex (Genepharma, Suzhou, China), MTS reagent (CellTiter 96 AQueous One Solution, Promega, Beijing, China, cat\# 3580), pfu High fidelity enzyme (Qiagen, Beijing, China, cat\# KP202), pJet1.2 vector (Thermo Fisher Scientific, Carlsbad, CA,USA, cat\# K1231), pGL6-basic (Beyotime Biotechnology, Haimen, China, cat\# D2105), pRL-TK Vector (Beyotime Biotechnology, Inc, cat\# D2762), a human pCMV-Nrf2 (Sino Biological, Beijing, China, cat\# HG17384-U), a mouse pCMV-Nrf2 (Sino Biological, Beijing, China, cat\# MG56971-UT), or an empty 
pCMV3 vector (Sino Biological, Beijing, China, cat\# D2602), mitochondrial extraction kit (Beyotime Biotechnology, cat\# C3601), 2',7'-Dichlorodihydrofluorescein diacetate (Thermo fisher, Shanghai, China, cat\# 2938), JC-1 probe solution (Sigma, St Louis, MO, USA, cat\# CS0760), Neuro-2a (N2A)( ATCC, Manassas, VA cat\# CCL-131, RRID:CVCL_0470), SK-N-BE(2) (ATCC Cat\# CRL-2271, RRID:CVCL_0528).

\section{Animal}

All mice were fed water and food ad libitum in a temperature- and humiditycontrolled animal facility with an automatic illumination on a 12-h on/off cycle in Wenzhou Medical University. APPswe/PSEN1dE9 mice (Jackson Laboratory, stock number 004462) express a K595N/M586L Swedish mutations and a mutant human presenilin 1 with deletion of exon9 under the control of mouse prion promoter. The mice were multiplied and genotyped according to the guidance by Jackson Laboratory. Both genders of 4- and 11-month-old transgenic mice were used and WT littermates were

used as controls. Behavioral experiments were performed during the daytime and at the same time period in each day.

\section{Intrahippocampal injection of adenovirus expressing Dicer1}

The adenovirus (Ad) incorporating sequences of either Ad-pCMV-EGFP or AdpCMV-Dicer1:T2A:EGFP in which the transcription of Dicer1 and EGFP is driven by independent promoter were packaged and generated by Cyagen Biosciences Inc. (Guangzhou, China). Before stereotactic injection, animals were anaesthetized by 
intraperitoneal injection of ketamine/xylazine $(0.1 / 0.05 \mathrm{mg} / \mathrm{g}$ body weight) and mounted on a stereotactic frame (KOPF, KD Scientific). The virus were injected into bilateral hippocampus with $2 \mu \mathrm{l}$ of viral titre $\left(1.2 \times 10^{9} \mathrm{vg} / \mathrm{mL}\right)$ in each hemisphere using a $10-\mu \mathrm{l}$ Hamilton syringe (Hamilton Medical, Reno, NV, USA) connected to a 30-gauge micropipette, at an injection rate of $0.2 \mu \mathrm{l} / \mathrm{min}$. The injection coordinates were anteriorposterior, $-2 \mathrm{~mm}$, mediolateral, $\pm 2 \mathrm{~mm}$, dorsoventral, $-2 \mathrm{~mm}$ from bregma. The person in charge of injection wore protective equipment and the injections were conducted in a biological safety cabinet in a biological safety level II lab setting. Autoclave of disposals was used to prevent contamination. The injections were conducted in 3.5-/4-month-old mice and behavior test was performed during 17-23 days after injection in which learning curve was acquired during 17-22 days and probe trial conducted at the 23rd day after injection. Halves of mice were used for immunohistochemistry and halves of them used for western blot.

\section{Morris Water Maze}

Water maze tests were performed using three groups of mice, WT mice (with intrahippocampal injection of Ad-EGFP) and APP/PS1 mice (intrahippocampal injection of Ad-EGFP or Ad-Dicer1-T2A:EGFP). The water maze (1.2-m diameter) was filled with water $\left(24^{\circ} \mathrm{C}\right)$ and made opaque by the addition of nontoxic white paint. The water maze was surrounded by a black curtain (placed $80 \mathrm{~cm}$ away) that held three salient visual cues. Initially, mice were randomly trained in four quadrants of the water maze, and were allowed a maximum of $60 \mathrm{~s}$ to find a hidden platform $(10-\mathrm{cm}$ diameter, $1 \mathrm{~cm}$ 
under the water surface). Mice were trained for 6 days (4 trials per day, 10-min interval between trial). On day 7 , mice were given a 60 -s probe test (scanning the platform) to test their spatial memory. SLY-WMS Morris Water Maze System (Beijing Sunny Instruments Co. Ltd) consisting of a frame grabber, a video camera, a water pool, and an analysis software was used to monitor the animal's swimming pattern, distance, speed, and the amount of time spent in each of the four quadrants.

\section{Cell culture}

Newly born mice were used for culturing dissociated hippocampal and cortical neurons (CNs and HNs, respectively). Hippocampal or cortical tissue was mechanically dissociated after digestion with $0.25 \%$ trypsin (Beyotime, Haimen, China) and $0.1 \%$ DNasel (Takara, Dalian, China) at $37^{\circ} \mathrm{C}$ for $15 \mathrm{~min}$. DMEM/F12 containing $10 \%$ fetal bovine serum (Gibco, Billings, MA,USA) was then added, and the cells were centrifuged at $120 \mathrm{~g}$ for $3 \mathrm{~min}$ and the supernatants were then plated on poly-D-lysine-coated $8 \mathrm{~cm}$ culture dishes in neurobasal medium supplemented with 2\% B27 (Invitrogen, Carlsbad, CA,USA) and $10 \mu \mathrm{M}$ cytosine arabinoside (Sigma-Aldrich, Saint Louis, MO, USA). After 24 hours, the culture medium was replaced with fresh neurobasal medium supplemented with 2\% B27 and the neuronal cultures were used for experiments after three days in vitro culture (DIV 3). Before transfection, the medium was replaced with DMEM/F12 containing $0.1 \%$ bovine serum albumin. After $24 \mathrm{~h}$ in culture, neurons were used for transfection, $A \beta 42$ oligomer treatment or adenovirus infection. N2A, SK-N-BE(2) 
or HEK 293T cells was cultured in Dulbecco's modified Eagle's Medium(DMEM) containing $10 \%$ Fetal bovine serum(FBS).

\section{Plasmid construction and Luciferase reporter assay}

With genomic DNA extracted from HEK 293T cells as a template, the region upstream of Dicer1 transcription start site (tss) (Entrez acc.no.:NM_177438.2) were amplified, producing an amplimer containing sequence from -1280 (relative to tss) to +3 (promoter 3), using forward primer CGACGCGTTTGAGATTGCGCCACTACATT and reverse primer CCCAAGCTTCCCAAGCTTCCCAAGCTTCTTTGTGTCC. Two more regions were amplified spanning -1121 or -896 to +3 by alternating forward primer CGACGCGTAGCCTGGGTGACAGAGCGAAACTCT (promoter 2) or CGACGCGTACCTGAGCTGGCTGGGACCCAGCATTTA (promoter 1), respectively. The bold letters indicated the predicted AREs sites with consensus sequence TGA(C/G)NNNGC. A sequence not containing AREs site was also amplified by changing a forward primer CGACGCGTGGGCAGTCAGAGAGAGAGGAAAGGAAGG spanning -581 to +3 (promoter 0 ). The region spanning individual ARE sequence was also amplified by using forward primer CGACGCGTAGGAGATCAAGACCATCCTGGG and reverse primer CCCAAGCTTTAGTGGCGCAATCTCAGCT for ARE3 (124 bp).

ARE2 (192 bp) was amplified using forward primer CGACGCGTCAGCCTGGGTGACAGAGCGAAAC and reverse primer CCCAAGCTTCTGGTCTGCAAGGCA. ARE1(185 bp) was amplified using forward primer CGGCACGCGTGAGCTGCCTTGTGACTTTGCCTTC and reverse primer 
CCCAAGCTTCGTCTTTCAACACTTGATCA . PCR products were amplified by pfu High fidelity enzyme and cloned into a pJet1.2 vector and then subcloned into pGL6basic containing firefly luciferase coding sequence. The constructs spanning tss were referred to as Dicer 1 promoter 3-Luc, promoter 2-Luc, promoter 1-Luc, and promoter 0Luc and the constructs containing individual AREs were referred to as ARE3-Luc, ARE2-Luc, and ARE1-Luc. ARPE1 in promoter 1-Luc was mutated with forward primer incorporating mutated base pair in bold letters GTACCTGGCTGGGACCCAGCATTTA and reverse primer CCCAAGC-TTCCCAAGCTTCTTTGTGTCC; promoter 1-Luc was used as the template. Using the resultant plasmid as the template, the forward primer incorporating Mlu I restrict site and the reverse primer containing HindllI restriction site were used to generate product, which was subcloned into PGL6-basic, namely, mutant promoter-1. The forward primer was CGACGCGTACCGTACCTGGCTGGGACCCAG CATTTA and the reverse primer was CCCAAGCTTCCCAAGCTTCCCAAGCTT CTTTGTGTCC with restriction sites underlined.

The HEK293T cells were seeded at a density of $3 \times 10^{4}$ cells/per 48 wells and transfected according to the manufacturer's protocol (Lipofectamine 2000, Invitrogen). The Renilla luciferase-containing reporter (pRL-TK Vector) was mixed with individual construct containing firefly luciferase, and a human pCMV-Nrf2 or an empty pCMV3 vector at a ratio of $1: 20: 20$, respectively. The relative luminescence unit $(R L U)$ of luciferase/renilla assay was measured per the manufacturer's protocol (Beyotime Biotechnology, cat\#RG027) by a plate reader (SpectraMax M5, Molecular devices, San Jose, CA, USA ). 


\section{Chromatin immunoprecipitation (CHIP) assay}

The CHIP assay kit (Beyotime Biotechnology, cat\# P2078) was used to examine the interaction between Nrf2 and Dicer1 promoter. All the procedures were performed according to the manufacturer's instructions. SK-N-BE cells were plated on 10-cm culture plate until $~ 70 \%$ confluency and added with $37 \%$ formaldehyde diluted to a $1 \%$ final concentration. To crosslink the protein-DNA complexes, the cells were incubated with formaldehyde on a shaker for $10 \mathrm{~min}$ at $37^{\circ} \mathrm{C}$. $1 \mathrm{X}$ glycine solution at a final dilution was added to quench the formaldehyde, which was rocked at room temperature for 5 min, and then the media were removed. The cells were immediately washed twice and harvested with ice-cold phosphate buffered saline (PBS) containing $1 \mathrm{mM}$ phenylmethylsulfonyl fluoride (PMSF), with $2 \times 10^{8}$ cells in each microcentrifuge tube. The harvested cells were subject to centrifuge with $1000 \times \mathrm{g}$ at $4{ }^{\circ} \mathrm{C}$, resuspended in SDS (sodium dodecyl sulfate) lysis buffer containing $1 \mathrm{mM} \mathrm{PMSF}$, and incubated on ice for $10 \mathrm{~min}$. Following the lysis, the samples were sonicated to shear chromatin, centrifuged for $5 \mathrm{~min}$ at $13,000 \mathrm{xg}$ at $4{ }^{\circ} \mathrm{C}$, and the supernatants were harvested and diluted 10 -fold in ChIP dilution buffer containing $1 \mathrm{mM} \mathrm{PMSF.} \mathrm{After} \mathrm{dilution,} 15 \%$ of each sample was collected to use as a positive input in PCR analysis. The rest of each sample ( $2 \mathrm{~mL}$ ) were pre-absorped with addition of $70 \mu \mathrm{L}$ protein $\mathrm{A}+\mathrm{G}$ agarose/salmon sperm DNA, and the mixture were rocked for $30 \mathrm{~min}$ at $4{ }^{\circ} \mathrm{C}$, centrifuged for $1 \mathrm{~min}$ at $1,000 \times \mathrm{g}$, and the supernatants were collected. After this pre-absorption, rabbit antiNrf2 antibody (Proteintech) was used to precipitate DNA-protein complexes; rabbit IgG (Beyotime Biotechnology, cat\#A7016) was used as a negative control. To capture the antibody, $60 \mu \mathrm{L}$ protein $\mathrm{A}+\mathrm{G}$ agarose/salmon sperm DNA was added, rocked for 60 min 
at $4{ }^{\circ} \mathrm{C}$, and centrifuged for $1 \mathrm{~min}$ at $1,000 \times \mathrm{g}$. The supernatants were discarded and the pellets were washed sequentially with Low, High Salt, and LiCl Immune Complex Wash buffer, and TE buffer. The pellets were used for PCR amplification and the following specific primers were used in PCR analyses for amplifying fragment containing ARE1 in Dicer1 promoter: sense, 5'-CACAGACCTGAGCTGGCTGGGAC-3'(-794 -817 relative to tss), antisense,5'- CAGATTGCGATGTGCGTGAAA-3'(-691 -712 relative to tss). For amplifying the fragment containing ARE2 in the promoter, the primers used were 5'-CAGCCTGGGTGACAGAGCGAAAC-3' (-1069 -1091 relative to tss) for sense, and 5'- CTGGTCTGCAAGGCAGTTTAT-3'(-876 -906 relative to tss) for antisense; for amplifying ARE3-containing fragment in the promoter, the primers were 5'AGGAGATCATGAGATTGCTGGG-3'(-1250 -1271 relative to tss) for sense, and 5'TAGTGGCGCAATCTCAGCT-3' (-1097 -1115 relative to tss) for antisense. DNA fragments from each group were amplified by PCR SuperMix(cat\# 10572014, Thermo Fisher) with an initial denaturation at $94^{\circ} \mathrm{C}$ for $5 \mathrm{~min}$, followed by 39 cycles ( $94^{\circ} \mathrm{C}$ for $30 \mathrm{~s}, 60^{\circ} \mathrm{C}$ for $45 \mathrm{~s}$, and $72^{\circ} \mathrm{C}$ for $20 \mathrm{~s}$ ). Finally, the reaction was ended with 5-min extension at $72^{\circ} \mathrm{C}$. The PCR products were assessed by use of $2 \%$ agarose electrophoretic gel.

\section{Aß42 oligomer preparation}

The oligomer form of $A \beta 42$ was prepared as described [26]. Briefly, the peptide was dissolved in 1,1,1,3,3,3-Hexafluoro-2-propanol (Sigma-Aldrich) to remove any aggregates, stored in $4^{\circ} \mathrm{C}$ for $30 \mathrm{~min}$, dried under room temperature, and dissolved in 
dimethyl sulfoxide (DMSO, Sigma-Aldrich) to $1 \mathrm{mM}$. The dissolved peptide was diluted in phenol-free $\mathrm{F} 12$ medium to $500 \mathrm{nM}$ and incubated at $4^{\circ} \mathrm{C}$ for $24 \mathrm{~h}$. The solution was subjected to centrifugation at $14,000 \times \mathrm{g}$ for $30 \mathrm{~min}$ at $4^{\circ} \mathrm{C}$. The supernatant was collected as the oligomer preparation which was used at a final concentration of $100 \mathrm{nM}$.

\section{Cell viability assay}

Neurons were cultured in 96 -well plates at a density of $5 \times 10^{3}$ per well in DMEM/F12 medium supplemented with B-27. After transfected with Dicer1 siRNA duplex (sense, 5'-GCACAUCAAGGUGCUACUATT-3', antisense, 5'UAGUAGCACCUUGAUGUGCTT-

3' ) or negative control siRNA duplex (sense, 5'-UUCUCCGAACGUGUCACGUTT-3', antisense, 5'-ACGUGACACGUUCGGAGAATT-3' ) by lipofectamine 2000 for $12 \mathrm{~h}$, the medium was replaced with fresh DMEM/F-12 medium and continued to culture for $36 \mathrm{~h}$. At the end of treatment, each well was added with $10 \mu \mathrm{L}$ MTS reagent $(500 \mu \mathrm{g} / \mathrm{mL})$ for 4 h. The absorption values were read in a plate reader at 490nm (SpectraMax M5, Molecular devices).

\section{Measurement of mitochondrial membrane potential and reactive oxygen species (ROS) activity}

Primary cultured cortical neurons at $9 \times 10^{5}$ cells/well and hippocampal neurons at $5 \times 10^{5}$ cells/ well were plated into 6 -well plates and cultured until DIV3. The neurons were transfected with Dicer1 duplex siRNAs ( $50 \mathrm{pM}$ ) per well by lipofectamine 2000 for 
$12 \mathrm{~h}$ in DMEM/F12 plus 2\% B27. After transfection, the cultured medium was replaced with fresh neurobasal medium supplemented with $2 \%$ B27 and continued to culture for $36 \mathrm{~h}$. For mitochondrial membrane potential $\left(\Delta \Psi_{\mathrm{m}}\right)$ assay, the neurons at the end of treatment were stained with $\mathrm{JC}-1$ probe solution at $37^{\circ} \mathrm{C}$ for 20 min and observed under fluorescence microscope (DMi8, Leica Biosystems, Wetzlar, Germany). The mitochondria were also extracted by a mitochondrial extraction kit. Neural mitochondrion $(30 \mu \mathrm{g})$ from each group was incubated with $\mathrm{JC}-1$ probe solution at $37^{\circ} \mathrm{C}$ for $20 \mathrm{~min}$. The reaction product was added into a 96-well plate for fluorescence measurement, which was read with excitation at $490 \mathrm{~nm}$ and emission at $520 \mathrm{~nm}$ for monomer (green) and with excitation at $530 \mathrm{~nm}$ and emission at $590 \mathrm{~nm}$ for aggregates (red). The $\Delta \Psi_{\mathrm{m}}$ values were represented by the ratios between aggregate and monomer fluorescence in relative fluorescence units ( RFU). For reactive oxygen species (ROS) assay, CNs and HNs were incubated with $10 \mu \mathrm{M} \mathrm{2',7'-}$

Dichlorodihydrofluorescein diacetate in neurobasal medium supplemented with $2 \%$ B27. After incubation at $37^{\circ} \mathrm{C}$ for $30 \mathrm{~min}$, the neurons were added into 96 -well plate at the density of $4 \times 10^{4}$ per well and subjected to measurement with plate fluorescence reader at $485 \mathrm{~nm}$ (SpectraMax M5, Molecular devices).

\section{Neurite outgrowth assay}

The neuronal cultures were treated by oligomer $\mathrm{A} \beta 42$ at $100 \mathrm{nM}$ for $24 \mathrm{~h}$ and then infected with Ad-EGFP or Ad-Dicer1-T2A:EGFP virus $\left(5 \times 10^{7} \mathrm{vg} / \mathrm{mL}\right)$ for $48 \mathrm{~h}$. For determination of neurite, the neurites at least two times longer than the soma diameter 
were included in the measurement (Image pro plus, Olympus Optical Co., Ltd, Tokyo, Japan). The measurement of neurite length was conducted in three independent culture preparation and the values were averaged from 150 infected neurons in either group.

\section{Western blot analysis}

The 4- or 11-month-old mice were fasted overnight, and at the next morning, mice were deeply anesthetized by isoflurane and transcardially perfused with ice-cold phosphate buffer (PBS). The hippocampi were isolated and freshly frozen in liquid nitrogen. For analyzing the neurons treated by amyloid peptide, the cytoplasmic and nuclear proteins were extracted by plasma and nuclear protein isolation kit (Cat\# P0027, Beyotime Biotechnology), respectively. For analyzing the cells for overexpressing Nrf2 plasmid, SK-N-BE (2) or N2A cells were seeded in 6-well plates at the density of $1 \times 10^{6}$ or $5 \times 10^{5}$ cells/well, respectively. Tissue or cell samples were homogenized in Super RIPA buffer composed of components in mM: 460 Tris- $\mathrm{HCl}, \mathrm{pH} 7.4,138 \mathrm{NaCl}, 1$ EDTA, 2.5 NaF, 2.5 Na3VO4, 1 phenylmethanesulfonylfluoride, 1 dithiothreitol, supplemented with $0.1 \%$ Nonidet $\mathrm{P}-40$, and $1 \mathrm{X}$ protease/ $1 \mathrm{X}$ phosphatase inhibitor cocktail (Sigma, St.Louis, MO). Proteins were resolved on 12\% SDS-PAGE gels (Bio-Rad) and transferred to nitrocellulose membranes (ThermoFisher). Membranes were blocked in blocking buffer (Beyotime Biotechnology) and then incubated with primary antibody. Following wash, the membranes were then incubated with secondary antibody, and 
developed with chemiluminescence system kit (ThermoFisher). The blots were quantified using Image $\mathrm{J}$ software (National Institutes of Health, Bethesda, MD, USA).

\section{Quantitative real-time polymerase chain reaction (qRT-PCR)}

The hippocampi were isolated and freshly frozen in liquid nitrogen. SK-N-BE cells or Neuro-2A cells were plated on 6 -well plate until $~ 70 \%$ confluency, and transfected with $1.5 \mu \mathrm{g} \mathrm{pCMV3} \mathrm{Nrf2} \mathrm{vector} \mathrm{or} \mathrm{pCMV} \mathrm{empty} \mathrm{vector} \mathrm{for} 48 \mathrm{~h}$ in DMEM supplemented with $10 \%$ FBS, respectively. Extraction of total RNAs from hippocampi or transfected cells and elimination of genomic DNA were performed by a RNA extraction kit (TIGEN BIOTECH, Co, LTD., Beijing, China, cat\# DP424). RNA (200 ng) was used to synthesize the cDNA by a first stand cDNA synthesis kit (cat\# R111-02, Vazyme, NanJing, China). Fifty nanograms of cDNA was used as a template to amplifying individual mRNA. The following primers were used in qRT-PCR: hDicer 1, sense, CCCGGCTGAGAGAACTTACG, antisense, CTGTAACTTCGACCAACACCTTTAAA; hNrf2, sense, TTCCCGGTCACATCGAGAG, antisense, 5'-TCCTGTTGCATACCGTC TAAATC-3'; mDicer1, sense, 5'-GTCAGCCGTCAGAACTCACTC-3', anti-sense, 5'ACAGTCAAGGCGACATAGCAA-3'; mNrf2, sense, 5'-CAGCATAGAGC AGGACATGGAG-3',antisense,5'- GAACAGCGGTAGTATCAGCCAG-3'; B1 RNA, sense, 5'-TGCCTTTAATCCCAGCACTT-3', anti-sense, 5'- GCTGCTCACA

CAAGGTTGAA-3'; the 18srRNA, sense, 5'-TTCGTATTGCGCCGCTAGA-3', antisense, 5'-CTTTCGCTCTGGTCCGTCTT-3'. The SYBR ${ }^{\mathrm{TM}}$ Green (Invitrogen) was used as the dye for the qRT-PCR reaction following the manufacturer's instruction. The 
amplification processes were initiated at $50^{\circ} \mathrm{C}$ for $2 \mathrm{~min}$ and a secondary step at $95^{\circ} \mathrm{C}$ for $10 \mathrm{~min}$, followed by 40 cycles of PCR reactions $\left(95^{\circ} \mathrm{C}\right.$ for $10 \mathrm{~s}$ and $60^{\circ} \mathrm{C}$ for $1 \mathrm{~min}$ ). Reaction specificity, indicated by the presence of a single amplification peak for each PCR reaction, was examined by use of dissociative curves. $2^{-\Delta \Delta}{ }^{\mathrm{Ct}}$ method was used to quantify the reaction, which was normalized to sham treatment or transfection of pCMV3 empty vector. Primary cultured cortical neurons and hippocampal neurons were plated on 6-well plate, and treated with $\mathrm{A} \beta 42$ oligomer (100 nM) or sham treatment for $48 \mathrm{~h}$ in neurobasal medium with $2 \% \mathrm{~B} 27$, respectively. Dicer1 mRNA levels or B1 RNA levels were assessed by qRT-PCR as above.

\section{Immunofluorescence}

The 4- or 11-month-old WT, APP/PS1 non-fasted mice were deeply anesthetized by isoflurane, killed, and then transcardially perfused with ice-cold PBS followed by $4 \%$ paraformaldehyde (PFA) in PBS. The isolated brains were postfixed in 4\% PFA overnight, then stored in PBS containing $0.01 \%$ sodium azide. Brains were mounted in agarose and $10 \mu \mathrm{m}$ coronal sections were obtained using a Leica VT1000S Vibratome. Antigen demasking was performed in $0.01 \mathrm{M}$ sodium citrate solution $(\mathrm{pH} 6.0)$ at $99^{\circ} \mathrm{C}$ for 40 min. After washing the sections in PBS three times, 5 min each, at room temperature (RT), the sections were blocked using 5\% normal donkey serum in PBS containing $0.2 \%$ Triton $\mathrm{X}-100$ for $1 \mathrm{~h}$ at RT. The sections were incubated with the rabbit anti-NeuN (1:800, Beyotime Biotechnology) or monoclonal anti-Dicer 1(1:300, SigmaAldrich) overnight at $4^{\circ} \mathrm{C}$. For mice injected with virus, the sections were incubated with 
anti-Dicer 1(1:300, Sigma-Aldrich) or anti-activated caspase 3 (1:300, Beyotime Biotechnology). After washing 3 times with PBS $(3 \times 5$ min at RT), sections were incubated with secondary antibodies: Alexa Fluor 594 goat anti-rabbit or Alexa Fluor 488 goat anti-mouse IgG (1:1000, Life Technologies Corporation, Carlsbad, CA, USA) for $1 \mathrm{~h}$ at $\mathrm{RT}$, then washed three times with PBS $(3 \times 5 \mathrm{~min}$ at $\mathrm{RT})$. The sections were then mounted on coverslips (Citotest Labware Manufacturing Co., Ltd, Jiangsu, China) and the images of CA3 hippocampus were obtained with a fluorescent microscope (DMi8, Leica Biosystems).

\section{Experimental design and statistical analysis}

During Water Maze test, mice were evenly allocated to each experimental group according to the gender and ages, and the tests were conducted in a blinded manner, that is, the investigator was blinded to the group allocation of the genotype or treatment during the experiment and when assessing all the results. Animal experiment sample size calculator: http://www.lasec.cuhk.edu.hk/sample-size-calculation.html was used to determine the sample sizes, assuming $40 \%$ difference in the mean and standard deviation of $20 \%$ at power $95 \%$ and type I error 0.01 [27]. Similarly, determination of neurite outgrowth was conducted in a blinded manner. All the data acquired during experiments were included for analysis and there were no sample size differences between the beginning and end of experiments. All data were presented as means \pm SD unless specified. Single data points were shown as overlaying dot-plot on bar-graph when sample sizes were smaller than 15, otherwise, shown as bar-graph. 
For parametric data, Student's t-test was used for comparing differences between two groups. In experiments with more than two groups, one-way ANOVA was performed followed by Tukey's post hoc test for comparisons among groups. For analysis of two groups of non-parametric data, the Mann-Whitney U-test was used for determining the differences. For Morris water maze experiment, two-way ANOVA with repeat measures followed by Tukey's post hoc test was used for analyzing the time spent in four quadrants, frequency to crossing platform, swimming speed and distance. Statistical analyses were performed with Graphpad Prism 7.04 software (Graphpad software, Inc. La Jolla, CA, USA). Difference was regarded as significance when $p<0.05$. 


\section{Results}

\section{Dicer1 or Nrf2 expression was reduced in the hippocampus of APP/PS1 mice}

Dicer1 is reduced in the RPE cells in advanced dry AMD[20] and knockout of Dicer1 induces neurodegeneration [22, 23]. In light of these evidence, we explored Dicer1 expression in the brain of the AD mice, APP/PS1. The staining of Dicer1 was reduced in the CA1, CA3, and dentate gyrus (DG) of APP/PS1 mice compared to WT littermate, at the age of 4 months or 11 months (Figure.1A). The protein was also significantly reduced in the homogenates from the hippocampus of 4-month APP/PS1 mice compared to WT littermate $(p=0.0092, n=6$ for each genotype, 3 female and 3 male) and this reduction became severe at the age of 11 months $(p=0.0002, n=6$ for each genotype, 3 female and 3 male) (Figures.1B and 1C). Consistent with reduced protein, Dicer1 mRNA was also reduced in the hippocampus compared to WT at the age of 4 months $(p=0.0335)$, and at the age of 11 months as well $(p=0.0304)$ (Figure.1F). Similarly, Dicer1 was significantly reduced in the homogenates from the parietal lobe of APP/PS1 mice at the age of 6 months when compared to WT littermate $(p=0.022, n=6$ for each genotype, 3 female and 3 male, $)($ Supplemental Figure.1). Nrf2 is a master regulator of anti-oxidation genes regulating redox homeostasis[28]; Dicer1 depletion induces cytotoxicity via oxidative stress[20]. Thus, we simultaneously examined the protein levels of Nrf2 and activated caspase 3 in the homogenates from APP/PS1 hippocampi. Similarly, Nrf2 protein levels in AD mice were significantly reduced compared to WT littermate at the age of 4 months $(p=0.0001)$ and 11 months $(p=0.0038)$, respectively (Figures. 1B and 1D). In accordance with these findings, Nrf2 mRNA in hippocampus from AD mice was reduced compared to WT littermate at 4 
months $(p=0.0167)$ and at 11 months as well $(p=0.0195)$, respectively (Figure. $1 G)$ Activated caspase 3 in AD mice was significantly increased compared to WT littermate at 4 months $(p=0.0098)$ and 11 months $(p=0.0008)$, respectively (Figures. $1 B$ and $1 E)$.

\section{Dicer1 knockdown induced neurotoxicity}

Dicer1 depletion induced cytotoxicity involving the roles of ROS and inflammation in the RPE cells [21]. Thus, we explored the relevant effects by knocking down Dicer1with specific siRNA in the primary murine hippocampal neuronal cultures (HNs). The Dicer1 siRNA used was selected from three siRNAs basing on efficacy (Supplemental Figure. 2A) and siRNA3 was used as Dicer1 siRNA in the following experiments. As expected,

Dicer1 knockdown by the specific siRNA induced ROS production in HNs $(p=0.00001)$ compared to NC siRNA (Figure. 2A). Similarly, Dicer1 knockdown reduced mitochondrial membrane potential indicated by JC- 1 staining in the $\mathrm{HNs}(p=0.0399)$ (Figures. 2B and 2C). We further explored the effect of Dicer1 knockdown on neuronal survival and the related signaling. Under the condition of Dicer1 knockdown, neuronal survival was compromised in the HNs $(p=0.0012)$ (Figure. 2D). The protein levels of activated caspase 3 under Dicer1 knockdown were also increased in the HNs $(p=0.020)$ compared to the effects of scrambled siRNA (NC siRNA) (Figures. 2E and 2G). Compared to transfection with NC siRNA, the Dicer1 siRNA significantly decreased Dicer1 protein in the HNs $(\mathrm{p}=0.044)$ (Figures. $2 \mathrm{E}$ and $2 \mathrm{~F})$. The effects of Dicer1 knockdown on ROS production, mitochondrial integrity and neuronal survival were also 
replicated in the primary murine cortical neuronal cultures (Supplemental Figures. 2B$2 \mathrm{H})$.

\section{Aß42 oligomer decreased Dicer1 and Nrf2 in neurons upon chronic exposure}

As above, we found that Dicer1 was reduced in APP/PS1 mice; the mice contain robust level of soluble $A \beta$ and the deletion mutation (dE9) significantly accelerates the shift of the $A \beta 40 / A \beta 42$ ratios and elevates the levels of $A \beta 42[29,30]$. Thus, we investigated the effect of $A \beta 42$ on Dicer1 expression in the CNs. Initially, we tested the time-course effect of $A \beta 42$ on Dicer1 or Nrf2, and $A \beta 42$ significantly reduced the protein levels of Dicer1 or Nrf2 after 12-48 h treatment but did not change Nrf2 protein level upon 6-h treatment (Figures. 3A-3C). A 342 oligomer also decreased Dicer1 mRNA in the CNs $(p=0.0277)$ or in the HNs $(p=0.0387)$ (Supplemental Figure. $3 A)$. To investigate the consequence of Dicer1 reduction by $A \beta 42$ oligomer, we examined one of Dicer1 substrates, B1 RNA (Alu-equivalent in rodents) in the CNs $(p=0.0469)$ or in the HNs $(p=0.0252)$ (Supplemental Figure 3B). We further tested whether reduction of

Dicer1 by $A \beta 42$ could be rescued by $\mathrm{N}$-acetyl-cysteine (NAC), an antioxidant. As expected, $\mathrm{A} \beta 42$ treatment for $48 \mathrm{~h}$ reduced Dicer1 in $\mathrm{CNs}$ compared to sham treatment $(p=0.00001)$, which was significantly rescued by NAC $(p=0.032)$ (Figures.3D and 3E). Simultaneously, we examined total Nrf2, which was also reduced by A $\beta 42(p=0.0005)$, the effect rescued by addition of NAC $(p=0.0009)$ (Figures. 3D and 3F). Since Nrf2 affects anti-oxidant gene transcription via interacting with AREs in nucleus [31], we further examined the levels of nucleic or cytosolic Nrf2 under A $A 42$ treatment or those 
under simultaneous addition of NAC. AB42 reduced either nucleic Nrf2 $(p=0.0326)$ (Figures. 3G and 3I) or cytosolic Nrf2 ( $p=0.0076)$ (Figures. 3Hand 3J), which was rescued by addition of NAC ( $p=0.00001$ for nucleic)or ( $p=0.0419$ for cytosolic), respectively (Figures. $3 \mathrm{G}$ and $3 \mathrm{H}$ ).

\section{Nrf2 regulated Dicer1 expression}

Nrf2 is a master regulator of transcription of anti-oxidant genes. We hypothesized that Nrf2 may regulate Dicer1 expression. To test this hypothesis, we examined Dicer1 expression in Neuro-2a cells with overexpression of Nrf2, compared to the cells transfected with vehicle plasmid. The levels of Dicer1 mRNA were increased by Nrf2 overexpression in Neuro-2a cells $(p=0.0016$ for Nrf2 mRNA; $p=0.0482$ for Dicer1 mRNA) (Figures. 4A and 4B). The protein levels of Dicer1 and Nrf2 were also examined by western blot. Consistent with increased levels of Dicer1 mRNA induced by Nrf2, overexpression of Nrf2 in Neuro-2a cells significantly increased Dicer1 protein when compared to the cells transfected with vehicle plasmid $(p=0.0004)$ (Figures. $4 \mathrm{C}-4 \mathrm{E})$. We next tested whether reduction of Dicer 1 by $A \beta 42$ could be rescued by overexpression of Nrf2. As expected, $A \beta 42$ decreased Dicer1 $(p=0.0004)$ which was rescued by Nrf2 overexpression in Neuro-2a cells $(\mathrm{p}=0.0399)$ (Figures. 4F-4H). We scanned the promoter of Dicer1 with software (promoter 2.0 prediction server) and found three AREs motif upstream of transcription start site (tss), that is, Dicer1 promoter region. We cloned the ARE1-ARE3-containing promoter fragments into PGL6-basic plasmid containing firefly luciferase-coding sequence (-Luc), respectively, and also cloned the 
promoters containing different ARE motif into PGL6-basic plasmid, respectively ( $p=0.00001$ ) (Figures. $4 \mathrm{I}$ and $4 \mathrm{~J})$. Transfection of Nrf2-expressing plasmid ( $\mathrm{pCMV} \mathrm{Nrf2)}$ increased the ratios between firefly and renilla in constructs containing ARE1 $(p=0.000219)$ or ARE3 motif $(p=0.043)$ when compared to the cells transfected with vehicle plasmid, pCMV3 (Figure. 4J). When compared the constructs containing different AREs under transfection with pCMV Nrf2, we found that the ratios of firefly/renilla from ARE1 constructs were significantly from the construct ARE2 $(p=0.000825)$, ARE3 $(p=0.0093)$, and PGL6-basic $(p=0.00041)$, respectively (Figure. $4 J)$. Transfection of pCMV Nrf2 increased firefly/renilla in promoter $1(p=0.00047)$, promoter $2(p=0.000135)$, promoter $3(p=0.000114)$, and mutant promoter $1(p=0.048)$ when compared to the cells transfected with pCMV3 (Figure. 4J). When compared different promoter constructs under transfection with PCMV Nrf2, we found that the ratios of firefly/renilla from promoter 1 were significantly from promoter $0(p=0.000361)$ and mutant promoter $1(p=0.009)$ but not different with promoter $2(p=0.194)$ or promoter 3 $(p=0.254)$ (Figure. 4J). To confirm whether Nrf2 directly interacted with AREs in the Dicer1 promoter, thereby regulating Dicer1 expression, we used Nrf2 antibody to capture Dicer1 promoter fragments and amplified the precipitated ARE1- (-871 -691 bp, relative to tss), ARE-2 (-1091 -876 bp), or ARE-3 (-1271 -1097 bp)-containing fragments, respectively in SK-N-BE cells (Figure. $4 \mathrm{I}$ and $4 \mathrm{~K}$ ). Basing on these data, we projected that Nrf2 regulated Dicer1 transcription via binding with AREs in the promoter in which the interaction between Nrf2 and ARE1 was most important for this regulation. Overexpression of Nrf2 increased Dicer1 was replicated in SK-N-BE cells as well (Supplemental Figure. 4) 


\section{Overexpression of Dicer1 recovered neurotoxicity by $A \beta 42$ oligomer}

Since we have demonstrated that Dicer1 depletion compromised cell survival (Figure 2), we wondered whether overexpression of Dicer1 mitigated A $\beta 42$-induced neurotoxicity. The infection rate of the virus reached $\sim 90 \%$ in the primary murine hippocampal neuronal cultures (Supplemental Figure 5). Aß42 oligomer treatment reduced the protein levels of Dicer1 compared to sham treatment $(p=0.033)$, which was rescued by infection with Ad-Dicer1-T2A:EGFP virus compared to infection with vehicle virus, Ad-EGFP ( $p=0.0074$ ) (Figures. 5A and 5B). A $A 42$ oligomer treatment increased activated caspase 3 compared to sham treatment $(p=0.0061)$, which was partially rescued by infection with Ad-Dicer1-T2A:EGFP virus compared to infection with AdEGFP ( $p=0.023$ ) (Figures. 5A and $5 C$ ). Under expectation, $A \beta 42$ oligomer decreased neuronal survival $(p=0.00067)$, which was partially rescued by infection with Ad-Dicer1T2A:EGFP virus $(p=0.047)$ (Figure. 5D). Dicer1 knockdown disrupted neurite in primary cortical neurons ( $\mathrm{p}=0.011$ ) ( Figures. $5 \mathrm{E}$ and $5 \mathrm{~F}$ ), which is reminiscent of the early axonal degeneration in AD. Similarly, $A \beta 42$ oligomer treatment trimmed neurite $(p=0.025)$, which was significantly recovered by overexpression of Ad-Dicer1T2A:EGFP virus (Figures. 5G-5J).

\section{Overexpression of Dicer1 improved spatial learning in APP/PS1 mice}

We further explored the effect of overexpression of Dicer1 in APP/PS1mice. Injection of virus induced reliable transfection (Supplemental Figure 6). Consistent with previous observation in Figure. 1A, Dicer1 staining was decreased in CA3 hippocampus 
compared to WT littermate at the age of 3.5 months; injection of Ad-Dicer1:T2A:EGFP significantly increased Dicer1 staining in CA3 hippocampus in APP/PS1 mice (Figure. 6A). CA3 hippocampi of APP/PS1mice contained stronger staining for activated caspase 3 compared to those of WT littermate, which was reduced by injection of AdDicer1:T2A:EGFP virus (Figure.6B). In parallel experiment, we isolated the whole hippocampi and subjected the homogenates to western blot against Dicer1 and caspase 3. Consistent with the immunofluorescence, there was lower level of Dicer1 in APP/ PS1 mice than WT $(p=0.0217)$ which was recovered by injection of AdDicer1:T2A:EGFP ( $p=0.0093)$ (Figures. 6C and 6D); there was higher level of activated caspase 3 in APP/PS1mice than WT ( $p=0.00681)$ which was reduced by injection of AdDicer1:T2A:EGFP ( $p=0.0067)$ (Figures. $6 \mathrm{C}$ and 6E). We further investigated whether overexpression of Dicer1 had effect on spatial learning and thus examined behavior with Morris Water Maze. During the training period on days 1-6 after virus injection, the latency to reach platform for APP/PS1 mice was significantly reduced compared to that of WT mice ( $p=0.0148$, two-way ANOVA) (Figure. $7 \mathrm{~A}$ ) which is consistent with previous reports that cognitive function is compromised at 3.5-4 months of APP/PS1 mice [32, 33]. This reduction was reversed by injection with Ad-Dicer1:T2A:EGFP virus compared to injection with Ad1-EGFP virus $(p=0.008)$ on day $5(p=0.0057)$, or $(p=0.00001)$ on day $6(p=0.0025)$ (Figure. $7 A)$. Under the condition of combining timepoint and treatment, the latency was significantly reversed by injection with Ad1Dicer1:T2A:EGFP virus $(p=0.0001)(F$ day $(5,174)=36.44, p=0.00001$; Ftreatment $(2,174)$ $=2.045, \mathrm{p}=0.0241 ;$ Finteraction $(75,174)=2.3, \mathrm{p}=0.0148$, two-way ANOVA) (Figure. $7 \mathrm{~A})$. On day 7 after a 60 -s probe test for searching platform, we monitored animal's 
swimming pattern, distance, speed, and the amount of time spent in each of the four quadrants. Without difference on swimming distance $(p=0.3141)$ (Figure. 7D) and swimming speed ( $p=0.0948$ ) (Figure. 7E), APP/PS1mice spent less time in target quadrant than WT mice ( $p=0.0023$ ) in probe trial test, which was reversed by injection with Ad-Dicer1:T2A:EGFP virus $(p=0.0087)\left(F(2,42)=5.43, p=0.0092 ; F_{\text {quadrant }}(3,116)=\right.$ 4.576, $p=0.1122 ; F_{\text {treatment }}(2,116)=0.1032, p=1 ; F_{\text {interaction }}(45,116)=1.334 . p=0.0046$, two-way ANOVA) (Figure. 7B). Similarly, the AD mice crossed the platform less frequently than WT mice $(p=0.039)$, but the chance was increased by injection with AdDicer1:T2A:EGFP virus $(p=0.043)$ (Figure. $7 C)$. 


\section{Discussion}

In our study, reduction of Dicer1 was detected as early as around 4 months in APP/PS1 mice in which A $\beta$ deposition begins in the brain at the age of 6 months [29]. Furthermore, knockdown of Dicer1 in cultured neurons induced neurite deficit before soma degeneration, and intrahippocampal injection of Dicer1-expressing virus improved spatial learning. These evidence suggest that reduction of Dicer1 is an early event in $A D$, possibly before overt $A \beta$ deposition and targeting Dicer1 may provide a new strategy for $A D$ therapy, with target at the early stage of this disorder.

The expression or activity of Dicer1 is delicately maintained with the regulation at transcriptional, post-transcriptional, and post-translational levels. For instance, SOX4, a transcriptional factor involved in embryonic development and cell fate determination, positively regulates Dicer1 transcription [34]. Similarly, a transcription factor linked with microphthalmia, melanocyte inducing transcription factor, increases its transcription during melanocyte differentiation [35]. At post-transcriptional level, microRNA such as let-7 miRNA or miR-292 regulates Dicer1 via targeting its coding region or 3'-UTR, respectively, thereby inhibiting its expression [36, 37]. At post-translation level, phosphorylation of Dicer1 at its RNase IIIb and double-stranded RNA-binding domains promotes its nuclear translocation and inhibits its function [38]--particularly, a phosphomimetic of phospho-serine by replacing serine 1836 with aspartate impairs Dicer1 function and causes postnatal lethality [39]. Distinct with these studies, we delineate a novel regulation of Dicer1, that is, by Keap1-ARE-Nrf2 pathway, which is unreported before. $A \beta 42$ reduced Dicer1 protein in neurons at $48 \mathrm{~h}$ treatment which was possibly due to reduced nuclear Nrf2 from chronic $A \beta$ treatment. By contrast, at 6 
$\mathrm{h}$ treatment, $\mathrm{A} \beta 42$ reduced Dicer1 to a significant difference but Nrf2 was unchanged which suggested that Dicer1 diminution by acute $A \beta$ treatment occurred at posttranslational level which was independent on Nrf2. In other words, dicer1 depletion induces oxidative stress causing neurotoxicity; chronic $A \beta$ treatment reduced Dicer1 which involved the roles of reduced Nrf2 whereas acute $A \beta$ treatment-- 6 h treatment-reduced Dicer1 which was independent on Nrf2 and possibly took place at posttranslational level.

Oxidative stress is thought to be central in the pathogenesis of AD, for example, oxidative stress exacerbates insulin resistance in $A D$ brain [40] and disrupts mitochondria [41], resulting in failure to produce sufficient ATP for $\mathrm{Na}^{+}-\mathrm{K}^{+}-\mathrm{ATPase}$ and $\mathrm{Na}^{+}-\mathrm{Ca}^{++}$exchanger in order to maintain ion homeostasis in neurons [42-44]. Dicer1 depletion may be an internal sources contributing to oxidative stress by leading to accumulation of Alu RNA [21]. Intrahippocampal injection of Nrf2-expressing lentivirus improves spatial learning in APP/PS1 mice [45] but Nrf2 promotes cancer cell proliferation [46]. As such, manipulation of Nrf2 expression may lead to undesired side effects although it indicates potential benefit in AD brain [45]. By contrast, appropriate expression of Dicer1 may be an alternative strategy to oppose oxidative stress or other pathogenic factors in $A D$ brain.

Dicer1 is ubiquitously expressed in the brain including in neurons, astroglia, and oligodendrocytes $[23,24,47]$. With immunofluorescence, we at least demonstrated that neuronal Dicer1 was decreased in AD hippocampus compared to that in littermate control mice. The glial Dicer1 in astrocytes or in oligodendrocyes was not investigated in this study. Thus, Dicer1 reduction detected from APP/PS1 brain homogenates and 
secondary neurodegeneration are the combinatory effects from neurons and glia.

Therefore, it is important to determine the percentage of Dicer1 reduction in different cell type in AD brain for therapy purpose.

Dicer1 is central to the processing from pre-microRNAs to mature microRNAs, which regulate neuronal survival and neuritogenesis [48, 49]. Deletion of functional Dicer1 has been found to induce accumulation of Alu RNA and thereby to activate NLRP3 inflammasome and to induce oxidative damages in advanced dry AMD [20, 21]. Conditional ablation of Dicer1 in oligodendrocytes induces neuronal impairment via demyelination, oxidative damage, inflammatory gliosis in mice [23]. There is also evidence showing that conditional knockout of Dicer1 in dopaminergic neurons of ventral midbrain leads to neuronal loss through inhibiting micoRNAs biosynthesis[50]. Similarly, blocking microRNAs biosynthesis by Dicer1 deletion disrupts morphogenesis in the cortex and in the hippocampus [22]. Apart from Alu RNA accumulation, microRNAs deficiency due to Dicer1 depletion may play unexpected roles in AD neurodegeneration due to mature miRNA deficiency [22, 47].

\section{Conclusions}

In this study, we indicated that Dicer1 was reduced in the hippocampus and in the cortex of APP/PS1 mice in vivo, and further showed that Dicer1 was reduced by A $\beta 42$ oligomer in vitro. Dicer1 depletion led to neurite deficit and apoptosis by stress responses including increased ROS production, activated caspase3, and disrupted mitochondrial membrane potential. We further indicated that Nrf2 regulated Dicer1 expression which was linked with Keap1-Nrf2-ARE signaling pathway. Overexpression 
of Dicer1 increased neuronal survival and recovered neurite deficit by $A \beta 42$ oligomer in vitro, and in vivo, Dicer1 overexpression in CA3 hippocampus improved spatial learning in APP/PS1 mice. Altogether, we reveal a novel role of Dicer1 in AD and a novel regulation of Dicer1. Our findings suggest Dicer1 may be a target in $A D$ therapy.

\section{Abbreviations}

AD, Alzheimer's disease; Nrf2, nuclear factor erythroid 2-related factor 2; ARE, antioxidant response element; miRNA, microRNA; KEAP1, Kelch-like ECH-associated protein1; RPE, retinal pigment epithelial cell; NLRP, nucleotide-binding oligomerization domain, Leucine rich Repeat and Pyrin domain containing.

\section{Declarations}

\section{Ethical approval and consent to participate}

All experiments and data reports followed the SfN Policy on Ethics and the study was approved by the Animal Care and Use Committee of Wenzhou Medical University (Approval number\# wydw2019-0141). This study did not use human samples or tissues.

\section{Availability of data and materials}

The data and materials are available from corresponding author on reasonable request. 


\title{
Consent for publication
}

All the authors and funding agencies agree with publication of this study.

\section{Conflict of Interests}

All the authors have found no conflict of interests to disclose.

\section{Funding}

This study is supported by Natural Science Foundation of Zhejiang Province (LY18H120003) and by funding (QTJ10001) from Wenzhou Medical University.

\author{
Authors' contributions \\ Wang $\mathrm{Y}$ and Lian $\mathrm{ML}$ conducted all the experiments, collected, and analyzed the \\ data and Wang $\mathrm{Y}$ also participated in writing; Zhou $\mathrm{J}$ initiated Dicer1 immunoblotting \\ with AD mouse tissues; Wu SZ conceived of and supervised this project, analyzed the \\ data, wrote the manuscript.
}

\section{Acknowledgements}

The authors appreciate the support from biomedical workstation of School of Optometry and Ophthalmology, Wenzhou Medical University, and support from animal facility in Wenzhou Medical University.

\section{Authors' information}

Wang $\mathrm{Y}$ and $\mathrm{Zhou} \mathrm{J}$ are assistant professors and work under the supervision of Wu SZ in School of Optometry and Ophthalmology, Wenzhou Medical University; Lian ML is a graduate student under the supervision of Wu SZ; Wu SZ is a full professor and a 
principle investigator in School of Optometry and Ophthalmology, Wenzhou Medical University. 


\section{References}

1. Hardy J, Selkoe DJ: The amyloid hypothesis of Alzheimer's disease: progress and problems on the road to therapeutics. Science 2002, 297:353-356.

2.Mattson MP: Pathways towards and away from Alzheimer's disease. Nature 2004, 430:631-639.

3.Halliwell B: Oxidative stress and neurodegeneration: where are we now? J Neurochem 2006, 97:1634-1658.

4.Markesbery WR: Oxidative stress hypothesis in Alzheimer's disease. Free Radic Biol Med 1997, 23:134-147.

5.Stockwell BR, Friedmann Angeli JP, Bayir H, Bush Al, Conrad M, Dixon SJ, Fulda S, Gascon S, Hatzios SK, Kagan VE, et al: Ferroptosis: A Regulated Cell Death Nexus Linking Metabolism, Redox Biology, and Disease. Cell 2017, 171:273-285.

6.Belaidi AA, Bush Al: Iron neurochemistry in Alzheimer's disease and Parkinson's disease: targets for therapeutics. J Neurochem 2016, 139 Suppl 1:179-197.

7.Mark RJ, Hensley K, Butterfield DA, Mattson MP: Amyloid beta-peptide impairs ionmotive ATPase activities: evidence for a role in loss of neuronal $\mathrm{Ca2}+$ homeostasis and cell death. J Neurosci 1995, 15:6239-6249.

8.Mark RJ, Pang Z, Geddes JW, Uchida K, Mattson MP: Amyloid beta-peptide impairs glucose transport in hippocampal and cortical neurons: involvement of membrane lipid peroxidation. J Neurosci 1997, 17:1046-1054.

9.Keller JN, Germeyer A, Begley JG, Mattson MP: 17Beta-estradiol attenuates oxidative impairment of synaptic $\mathrm{Na}+/ \mathrm{K}+-\mathrm{ATP}$ ase activity, glucose transport, and glutamate transport induced by amyloid beta-peptide and iron. J Neurosci Res 1997, 50:522-530. 10.McWalter GK, Higgins LG, McLellan LI, Henderson CJ, Song L, Thornalley PJ, Itoh $\mathrm{K}$, Yamamoto M, Hayes JD: Transcription factor Nrf2 is essential for induction of $\mathrm{NAD}(\mathrm{P}) \mathrm{H}$ :quinone oxidoreductase 1, glutathione S-transferases, and glutamate cysteine ligase by broccoli seeds and isothiocyanates. The Journal of nutrition 2004, 134:3499S3506S.

11.Chen ZH, Saito Y, Yoshida Y, Sekine A, Noguchi N, Niki E: 4-Hydroxynonenal induces adaptive response and enhances PC12 cell tolerance primarily through induction of thioredoxin reductase 1 via activation of Nrf2. J Biol Chem 2005, 280:41921-41927.

12.Kobayashi A, Kang Ml, Okawa H, Ohtsuji M, Zenke Y, Chiba T, Igarashi K, Yamamoto M: Oxidative stress sensor Keap1 functions as an adaptor for Cul3-based E3 ligase to regulate proteasomal degradation of Nrf2. Mol Cell Biol 2004, 24:71307139.

13.Kurzynska-Kokorniak A, Koralewska N, Pokornowska M, Urbanowicz A, Tworak A, Mickiewicz A, Figlerowicz M: The many faces of Dicer: the complexity of the mechanisms regulating Dicer gene expression and enzyme activities. Nucleic Acids Res, 43:4365-4380.

14.Bernstein E, Caudy AA, Hammond SM, Hannon GJ: Role for a bidentate ribonuclease in the initiation step of RNA interference. Nature 2001, 409:363-366.

15.Kanellopoulou C, Muljo SA, Kung AL, Ganesan S, Drapkin R, Jenuwein T, Livingston DM, Rajewsky K: Dicer-deficient mouse embryonic stem cells are defective in differentiation and centromeric silencing. Genes Dev 2005, 19:489-501. 
16. Kota SK, Roy Chowdhury D, Rao LK, Padmalatha V, Singh L, Bhadra U: Uncoupling of X-linked gene silencing from XIST binding by DICER 1 and chromatin modulation on human inactive X chromosome. Chromosoma 2014, 124:249-262.

17.Alemdehy MF, van Boxtel NG, de Looper HW, van den Berge IJ, Sanders MA, Cupedo T, Touw IP, Erkeland SJ: Dicer1 deletion in myeloid-committed progenitors causes neutrophil dysplasia and blocks macrophage/dendritic cell development in mice. Blood 2012, 119:4723-4730.

18.Dincbas-Renqvist V, Pepin G, Rakonjac M, Plante I, Ouellet DL, Hermansson A, Goulet I, Doucet J, Samuelsson B, Radmark O, Provost P: Human Dicer C-terminus functions as a 5-lipoxygenase binding domain. Biochim Biophys Acta 2009, 1789:99108.

19.Nakagawa A, Shi Y, Kage-Nakadai E, Mitani S, Xue D: Caspase-dependent conversion of Dicer ribonuclease into a death-promoting deoxyribonuclease. Science 2010, 328:327-334.

20.Kaneko H, Dridi S, Tarallo V, Gelfand BD, Fowler BJ, Cho WG, Kleinman ME, Ponicsan SL, Hauswirth WW, Chiodo VA, et al: DICER1 deficit induces Alu RNA toxicity in age-related macular degeneration. Nature 2011, 471:325-330.

21.Tarallo V, Hirano Y, Gelfand BD, Dridi S, Kerur N, Kim Y, Cho WG, Kaneko H, Fowler BJ, Bogdanovich S, et al: DICER1 loss and Alu RNA induce age-related macular degeneration via the NLRP3 inflammasome and MyD88. Cell 2012, 149:847-859. 22.Davis TH, Cuellar TL, Koch SM, Barker AJ, Harfe BD, McManus MT, Ullian EM: Conditional loss of Dicer disrupts cellular and tissue morphogenesis in the cortex and hippocampus. J Neurosci 2008, 28:4322-4330.

23. Shin D, Shin JY, McManus MT, Ptacek LJ, Fu YH: Dicer ablation in oligodendrocytes provokes neuronal impairment in mice. Ann Neurol 2009, 66:843-857.

24.Tao J, Wu H, Lin Q, Wei W, Lu XH, Cantle JP, Ao Y, Olsen RW, Yang XW, Mody I, et al: Deletion of astroglial Dicer causes non-cell-autonomous neuronal dysfunction and degeneration. J Neurosci 2011, 31:8306-8319.

25.Varol D, Mildner A, Blank T, Shemer A, Barashi N, Yona S, David E, Boura-Halfon S, Segal-Hayoun Y, Chappell-Maor L, et al: Dicer Deficiency Differentially Impacts Microglia of the Developing and Adult Brain. Immunity 2017, 46:1030-1044 e1038. 26. Rushworth JV, Griffiths HH, Watt NT, Hooper NM: Prion protein-mediated toxicity of amyloid-beta oligomers requires lipid rafts and the transmembrane LRP1. J Biol Chem 2013, 288:8935-8951.

27.Jiang H, Wu M, Liu Y, Song L, Li S, Wang X, Zhang YF, Fang J, Wu S: Serine racemase deficiency attenuates choroidal neovascularization and reduces nitric oxide and VEGF levels by retinal pigment epithelial cells. J Neurochem 2017, 143:375-388. 28.Rada P, Rojo AI, Evrard-Todeschi N, Innamorato NG, Cotte A, Jaworski T, TobonVelasco JC, Devijver H, Garcia-Mayoral MF, Van Leuven F, et al: Structural and functional characterization of Nrf2 degradation by the glycogen synthase kinase 3/betaTrCP axis. Mol Cell Biol 2012, 32:3486-3499.

29.Jankowsky JL, Fadale DJ, Anderson J, Xu GM, Gonzales V, Jenkins NA, Copeland NG, Lee MK, Younkin LH, Wagner SL, et al: Mutant presenilins specifically elevate the levels of the 42 residue beta-amyloid peptide in vivo: evidence for augmentation of a 42 specific gamma secretase. Hum Mol Genet 2004, 13:159-170. 
30.Choi SH, Leight SN, Lee VM, Li T, Wong PC, Johnson JA, Saraiva MJ, Sisodia SS: Accelerated Abeta deposition in APPswe/PS1deltaE9 mice with hemizygous deletions of TTR (transthyretin). J Neurosci 2007, 27:7006-7010.

31. Itoh K, Tong KI, Yamamoto M: Molecular mechanism activating Nrf2-Keap1 pathway in regulation of adaptive response to electrophiles. Free Radic Biol Med 2004, 36:12081213.

32.Edwards SR, Hamlin AS, Marks N, Coulson EJ, Smith MT: Comparative studies using the Morris water maze to assess spatial memory deficits in two transgenic mouse models of Alzheimer's disease. Clin Exp Pharmacol Physiol 2014, 41:798-806.

33. Huang S, Cao X, Zhou Y, Shi F, Xin S, He S, An Y, Gao L, Yang Y, Yu B, Pei G: An analog derived from phenylpropanoids ameliorates Alzheimer's disease-like pathology and protects mitochondrial function. Neurobiol Aging 2019, 80:187-195. 34.Jafarnejad SM, Ardekani GS, Ghaffari M, Martinka M, Li G: Sox4-mediated Dicer expression is critical for suppression of melanoma cell invasion. Oncogene, 32:21312139.

35.Levy C, Khaled M, Robinson KC, Veguilla RA, Chen PH, Yokoyama S, Makino E, Lu J, Larue L, Beermann F, et al: Lineage-specific transcriptional regulation of DICER by MITF in melanocytes. Cell, 141:994-1005.

36.Forman JJ, Legesse-Miller A, Coller HA: A search for conserved sequences in coding regions reveals that the let-7 microRNA targets Dicer within its coding sequence. Proc Natl Acad Sci U S A 2008, 105:14879-14884.

37.Feinberg-Gorenshtein G, Guedj A, Shichrur K, Jeison M, Luria D, Kodman Y, Ash S, Feinmesser M, Edry L, Shomron N, et al: MiR-192 directly binds and regulates Dicer1 expression in neuroblastoma. PLoS One, 8:e78713.

38.Drake M, Furuta T, Suen KM, Gonzalez G, Liu B, Kalia A, Ladbury JE, Fire AZ, Skeath JB, Arur S: A requirement for ERK-dependent Dicer phosphorylation in coordinating oocyte-to-embryo transition in C. elegans. Dev Cell, 31:614-628.

39.Aryal NK, Pant V, Wasylishen AR, Parker-Thornburg J, Baseler L, El-Naggar AK, Liu B, Kalia A, Lozano G, Arur S: Constitutive Dicer1 phosphorylation accelerates metabolism and aging in vivo. Proc Natl Acad Sci U S A, 116:960-969.

40.De Felice FG, Lourenco MV, Ferreira ST: How does brain insulin resistance develop in Alzheimer's disease? Alzheimer's \& dementia : the journal of the Alzheimer's Association 2014, 10:S26-32.

41.Lin MT, Beal MF: Mitochondrial dysfunction and oxidative stress in neurodegenerative diseases. Nature 2006, 443:787-795.

42.Gloor SM: Relevance of Na,K-ATPase to local extracellular potassium homeostasis and modulation of synaptic transmission. FEBS Lett 1997, 412:1-4.

43.Reeves JP, Bailey CA, Hale CC: Redox modification of sodium-calcium exchange activity in cardiac sarcolemmal vesicles. J Biol Chem 1986, 261:4948-4955. 44.Kip SN, Strehler EE: Rapid downregulation of NCX and PMCA in hippocampal neurons following H2O2 oxidative stress. Ann N Y Acad Sci 2007, 1099:436-439. 45.Kanninen K, Heikkinen R, Malm T, Rolova T, Kuhmonen S, Leinonen H, YlaHerttuala S, Tanila H, Levonen AL, Koistinaho M, Koistinaho J: Intrahippocampal injection of a lentiviral vector expressing Nrf2 improves spatial learning in a mouse model of Alzheimer's disease. Proc Natl Acad Sci U S A 2009, 106:16505-16510. 
46.Jaramillo MC, Zhang DD: The emerging role of the Nrf2-Keap1 signaling pathway in cancer. Genes Dev 2013, 27:2179-2191.

47.Cuellar TL, Davis TH, Nelson PT, Loeb GB, Harfe BD, Ullian E, McManus MT: Dicer loss in striatal neurons produces behavioral and neuroanatomical phenotypes in the absence of neurodegeneration. Proc Natl Acad Sci U S A 2008, 105:5614-5619. 48. Im HI, Kenny PJ: MicroRNAs in neuronal function and dysfunction. Trends in neurosciences 2012, 35:325-334.

49.Carrella S, D'Agostino Y, Barbato S, Huber-Reggi SP, Salierno FG, Manfredi A, Neuhauss SC, Banfi S, Conte I: miR-181a/b control the assembly of visual circuitry by regulating retinal axon specification and growth. Developmental neurobiology 2015, 75:1252-1267.

50.Chmielarz P, Konovalova J, Najam SS, Alter H, Piepponen TP, Erfle H, Sonntag KC, Schutz G, Vinnikov IA, Domanskyi A: Dicer and microRNAs protect adult dopamine neurons. Cell Death Dis 2017, 8:e2813. 
Figures and figure legends
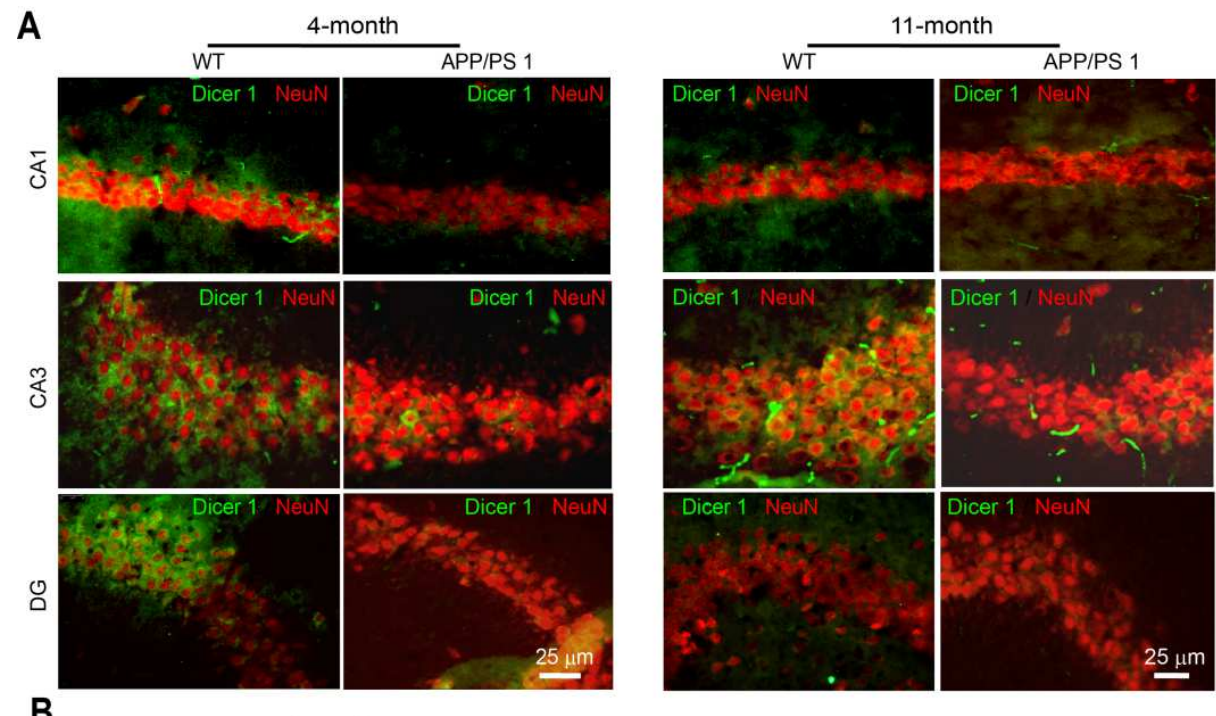

B

-month
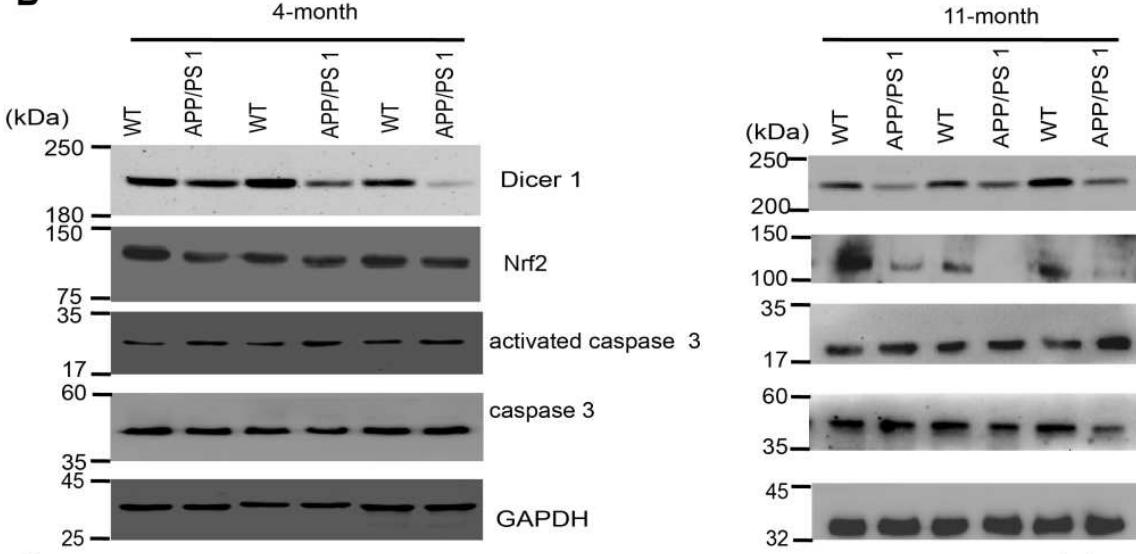

C

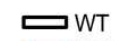

D चWT

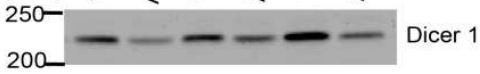

$200-\frac{-}{150-}-$ Dicer 1
$100-$
Nar2

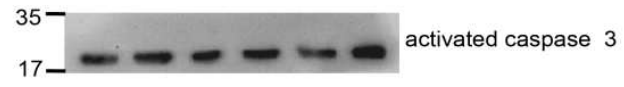

$60-$

35- ---- caspase 3
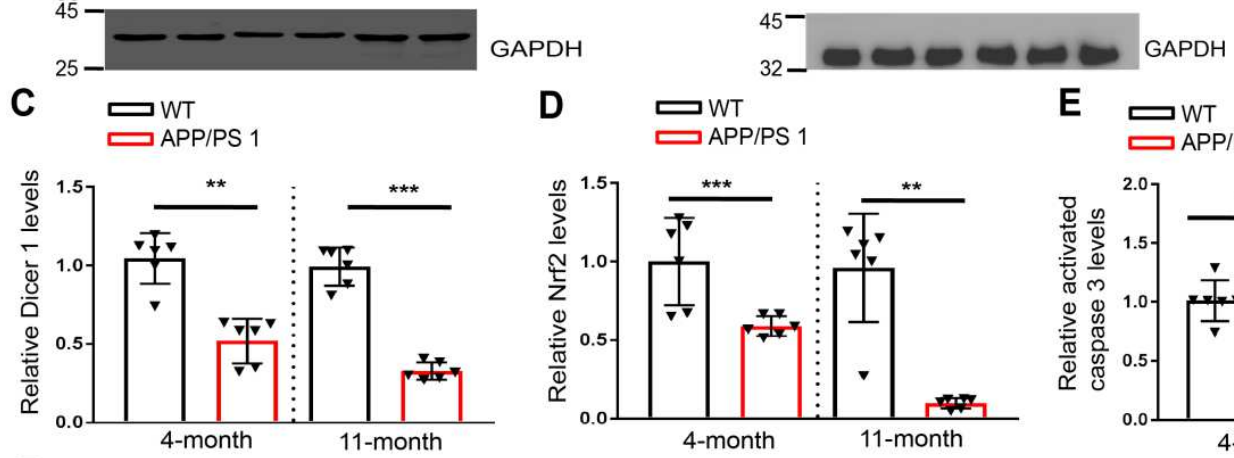

E 2 WT

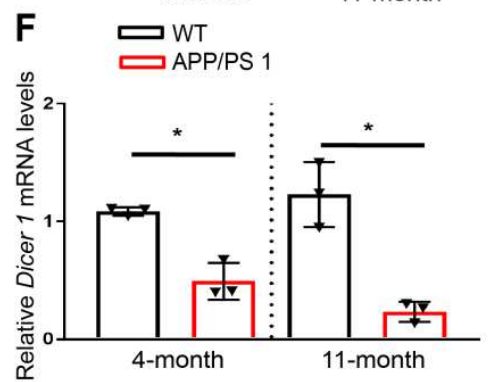

G 口WT
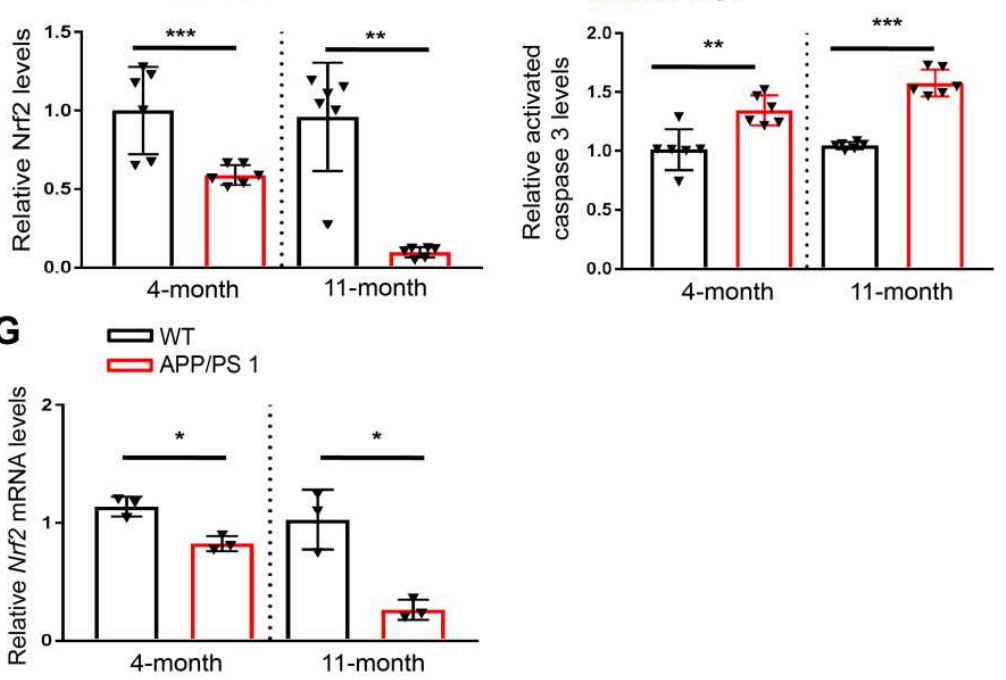
Figure 1. Reduction of Dicer 1, decreased Nrf2 and increased activated caspase-3 in the hippocampus of APP/PS1 mice. (A) APP/PS1 brains were processed into sections which were doubly stained for Dicer1 (green) or NeuN (red). The images were typical staining of CA1, CA3, or DG ( $\mathrm{n}=3$ mice per genotype). Bar, $25 \mu \mathrm{m}$ for all panels. (B) The supernatants from homogenized hippocampai at the age of 4-/11 months were collected and subject to examination of Dicer1, Nrf2, caspase-3, and activated caspase- 3 by western blot. GAPDH was used as a loading control. The protein levels of Dicer1 and Nrf2 in the hippocampal tissues were reduced in APP/PS1 mice ( $n=6,3$ male and 3 female) compared to WT littermate ( $n=6,3$ male and 3 female), respectively, at the age of 4 months (Left panel) or at the age of 11 months (right panel). By contrast, the protein levels of activated caspase-3 were increased in APP/PS1 mice $(n=6)$ compared to WT $(n=6)$ at the age of 4-/11 months. (C) The optical densities of Dicer1 relative to GAPDH were normalized to WT at the age of 4 months $\left({ }^{* *} \mathrm{p}=0.0092\right)$ or 11 months $\left({ }^{* * *} \mathrm{p}=0.0002\right)$ ( $\mathrm{n}=6$ per genotype, Mann-Whitney $U$ test). (D) Nrf2 relative to GAPDH were normalized to WT (Mann-Whitney $U$ test,${ }^{* \star *} \mathrm{p}=0.0001$ in 4-month, ${ }^{* *} \mathrm{p}=0.0038$ in 11 -month, $\mathrm{n}=6$ per genotype). (E) Activated caspase 3 relative to GAPDH were normalized to WT (Mann-Whitney $U$ test, ${ }^{* *} \mathrm{p}=0.0098$ in 4-month, ${ }^{* \star *} \mathrm{p}=0.0008$ in 11 -month, $\mathrm{n}=6$ per genotype), respectively. (F) Dicer1 mRNA was examined in WT $(n=3)$ and in APP/PS1 mice $(n=3)$ at the age of 4 months $(p=0.0335)$ or 11 months $(\mathrm{p}=0.0304)$ (paired $t$-test). (G) Nrf2 mRNA was examine in WT $(n=3)$ and in APP/PS1 mice $(n=3)$ at the age of 4 months $(p=0.0167)$ or 11 months $(p=0.0195)$ (paired $t$-test). 
A

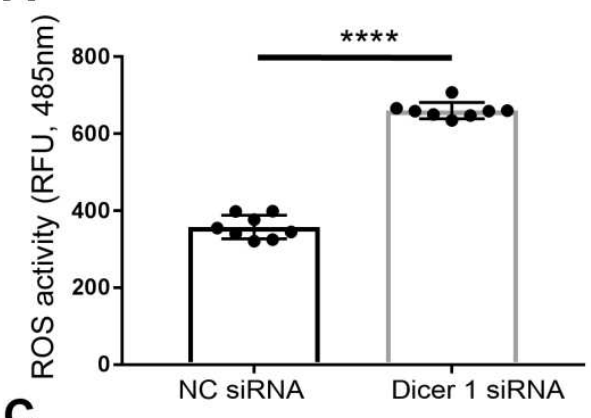

C

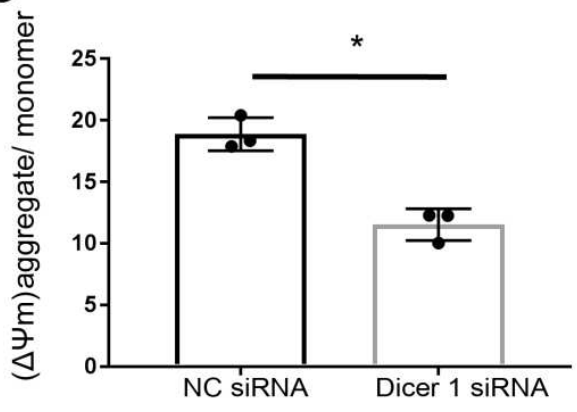

E

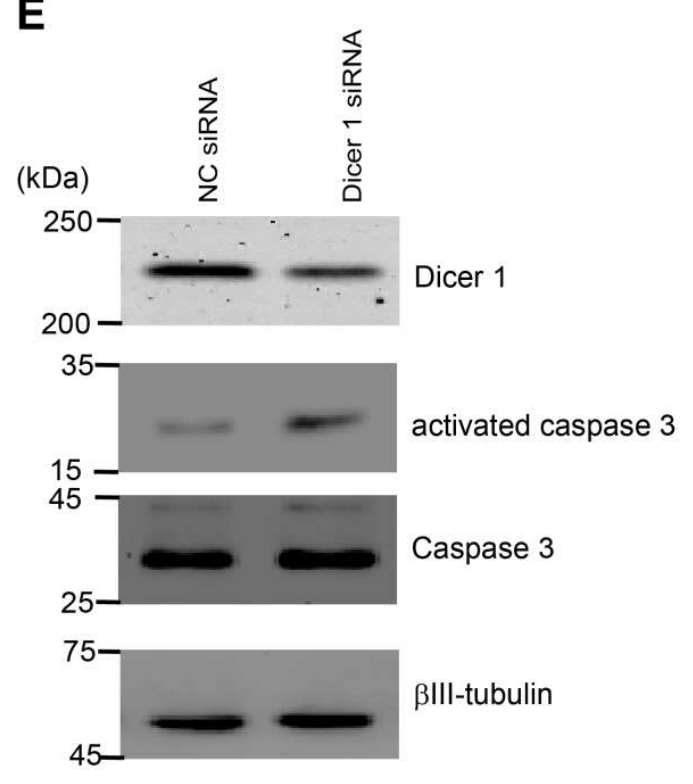

B

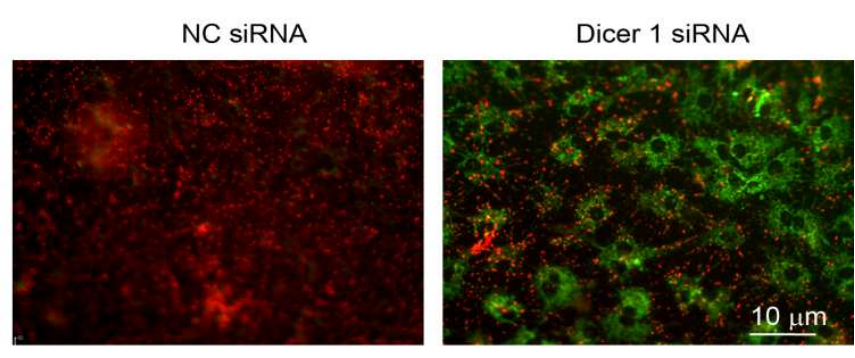

D

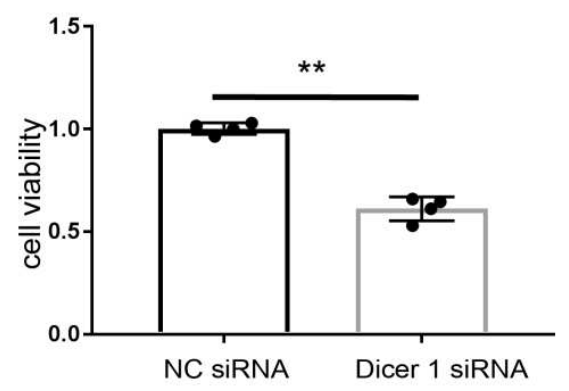

$\mathbf{F}$
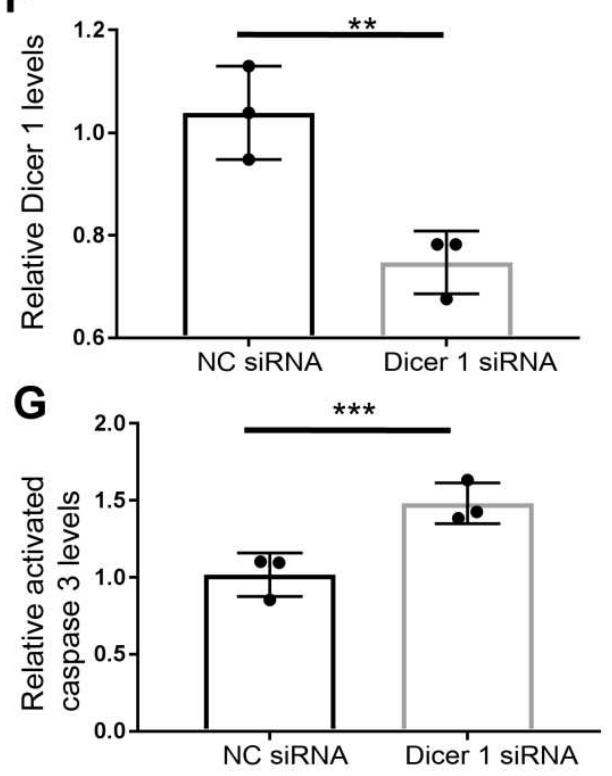

Figure 2. knocking down Dicer 1 induced production of reactive oxygen species (ROS), reduced mitochondrial membrane potential, enhanced apoptosis in primary murine hippocampal neuronal cultures. (A) The ROS levels in hippocampal neuronal cultures ( HNs) were measured by incubating 2',7'-dichlorodihydrofluorescein diacetate for $20 \mathrm{~min}$ at $37^{\circ} \mathrm{C}$. The fluorescence values in relative fluorescence units 
(RFU) were acquired in a plate reader at $485 \mathrm{~nm}$ and were averaged from four independent culture preparation with duplicate cultures in each preparation.

${ }^{* * * *} p=0.00001$. (B) Representative images of JC-1 staining inHNs subject to Dicer1 knockdown as above. Red fluorescence represented aggregate in mitochondria at hyperpolarized membrane potentials under healthy condition whereas green fluorescence as monomer in cytoplasm at depolarized membrane potentials under the context of compromised mitochondria. Bar $10 \mu \mathrm{m}$ for all panels. (C) Mitochondrial membrane potential $\left(\triangle \Psi_{\mathrm{m}}\right)$ of HNs subject to Dicer1 knockdown. The neurons were subject to Dicer1 knockdown and mitochondria $(30 \mu \mathrm{g})$ were isolated and stained with JC-1. The values of mitochondrial membrane potential $(\Delta \Psi \mathrm{m})$ were indicated by the ratios between aggregated/monomer RFU and averaged from three independent culture preparations. Student's $t$-test was used to compare the differences, ${ }^{*} \mathrm{p}=0.0399$. (D) Viability of neurons subjected to Dicer1 knockdown. HNs were transfected with Dicer 1 siRNA or NC siRNA as described in the materials and methods. The absorption values at OD $450 \mathrm{~nm}$ in Dicer1 siRNA group were measured and normalized to NC siRNA group. Student's $t$-test was used to compare the differences, ${ }^{* *} \mathrm{p}=0.0012$ for comparison . (E) HNs were transfected with Dicer1 siRNA (50 pM) or control siRNA duplex (50 pM) per well and subjected to western blot examination of Dicer1, activated caspase3, caspase 3 and $\beta$ III-tubulin was used as a loading control. (F) The effects of knockdown by Dicer1 siRNA were normalized to those of NC siRNA and averaged from three independent culture preparation. Student's $t$-test was used to compare the differences, ${ }^{*} \mathrm{p}=0.044$ for comparison. (G) The optical densities of activated caspase- 3 relative to $\beta$ III-tubulin from $F$ were normalized to NC siRNA group, and averaged from 
three independent culture preparation. Student's $t$-test was used to compare the differences, ${ }^{*} \mathrm{p}=0.02$ for comparison. 
A

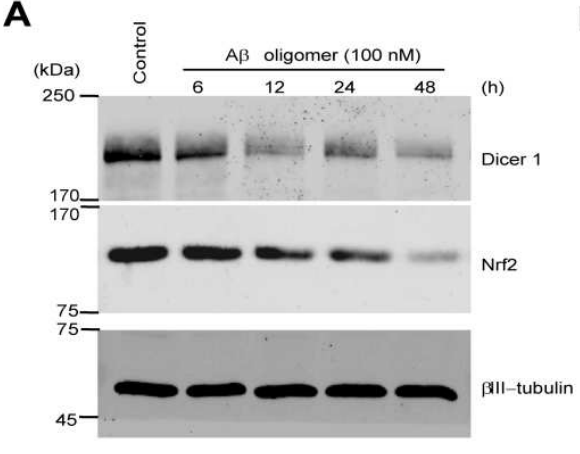

D

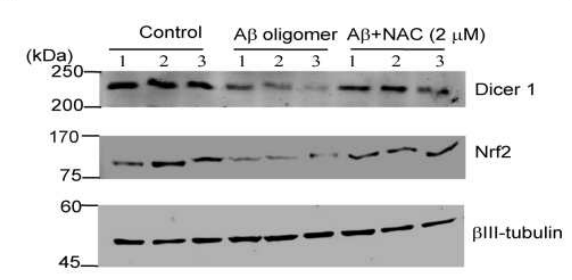

G
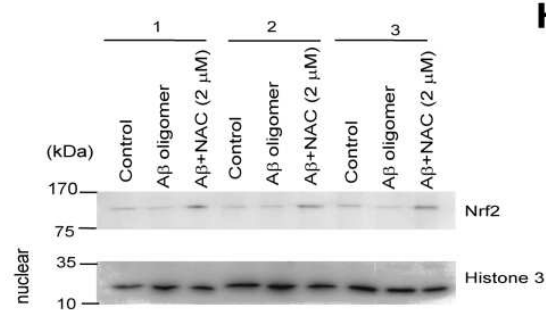

I
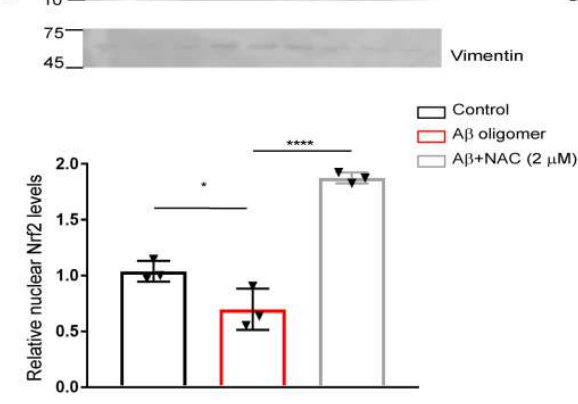

B

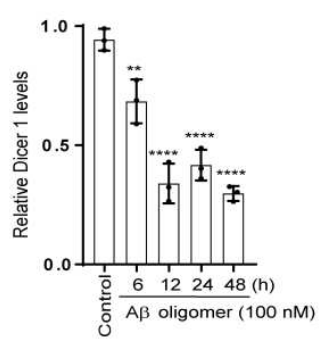

E

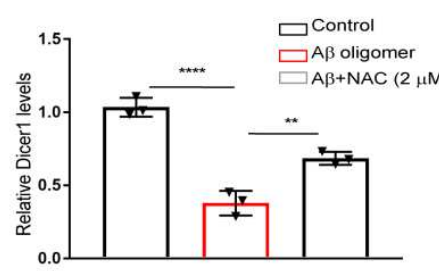

H

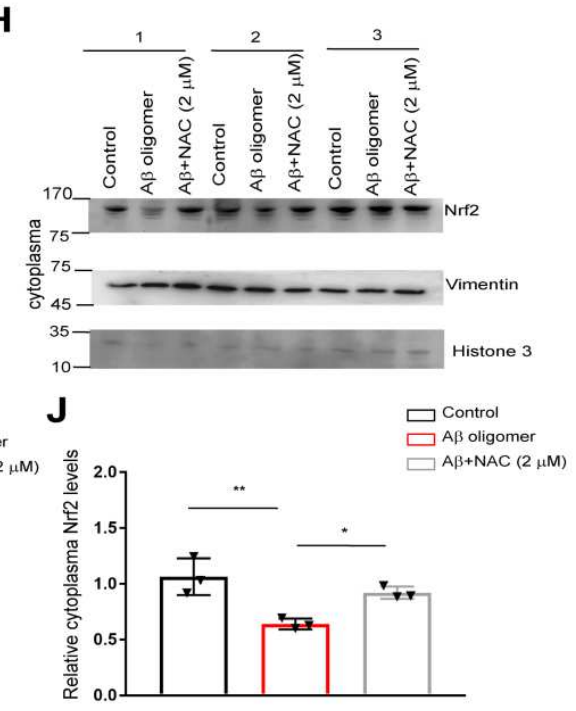

Figure 3. Chronic AB42 oligomer treatment reduced Dicer1 in neurons which was coupled with Nrf2 reduction, the effects rescued by NAC. (A-C) The time-course effect of $A \beta 42$ on Dicer1 or on Nrf2. The primary murine cortical neuronal cultures were subjected to vehicle or $A \beta$ oligomer treatment at indicated time. The cells were harvested for western blot for Dicer1 or Nrf2 and $\beta$ III-tubulin was used as a loading 
control. The relative optical densities of Dicer1 or Nrf2 from triplicate experiments were quantified and indicated in B and C. ${ }^{* *} \mathrm{p}<0.01,{ }^{* * *} \mathrm{p}<0.001,{ }^{* * *} \mathrm{p}<0.0001$, n.s indicated no significance. (D) The primary mouse cortical neurons were treated with $A \beta 42$ oligomer $(100 \mathrm{nM})$ and antioxidant NAC $(2 \mu \mathrm{M})$ for $48 \mathrm{~h}$. The neuronal cultures were harvested for western blot against Dicer 1, Nrf2, and Keap1. BIII-tubulin was used as an internal loading control. (E) The optical densities of Dicer1/ßIII-tubulin from D were normalized to sham treatment and averaged from three independent culture preparation. One-way ANOVA followed by Tukey's post hoc test was used to compare the differences. Control vs. $A \beta 42$ oligomer treatment, ${ }^{* * *} p=0.00001 ; A \beta 42$ oligomer vs. $A \beta 42$ oligomer plus $N A C$ treatment $(A \beta+N A C),{ }^{*} p=0.032$. (F) The optical densities of Nrf2/ßIII-tubulin from D were normalized to sham treatment and averaged from three independent culture preparation. One-way ANOVA followed by Tukey's post hoc test was used to compare the differences. Control vs. A 342 oligomer treatment, ${ }^{* * *} p=0.0005 ; A \beta 42$ oligomer vs. $A \beta+N A C,{ }^{* * *} p=0.0009$. (G) The neurons were treated with $A \beta 42$ oligomer $(100 \mathrm{nM})$ and antioxidant NAC $(2 \mu \mathrm{M})$ for $24 \mathrm{~h}$ and the nucleic proteins were extracted and blotted against Nrf2, and histone3 served as a loading control and vimentin as a negative control; $\mathbf{( H )}$ the cytoplasmic proteins were isolated and blotted against Nrf2, and vimentin served as a loading control and histone3 as a negative control. (I) The optical densities of nucleic Nrf2 relative to histone 3 averaged from three independent culture preparation in G. One-way ANOVA followed by Tukey's post hoc test was used to compare the differences. Control vs. $A \beta 42,{ }^{*} \mathrm{p}=0.0326 ; A \beta 42$ vs. $A \beta+N A C,{ }^{* * * *} p=0.00001$. (J) The optical densities of cytosolic Nrf2/vimentin were normalized to sham treatment and averaged from three independent culture preparation 
in $\mathrm{H}$. One-way ANOVA followed by Tukey's post hoc test was used to compare the differences. Control vs. $A \beta 42$ oligomer, ${ }^{*} \mathrm{p}=0.0076$; $A \beta 42$ oligomer vs. $A \beta+N A C$, ${ }^{*} p=0.0419$. 

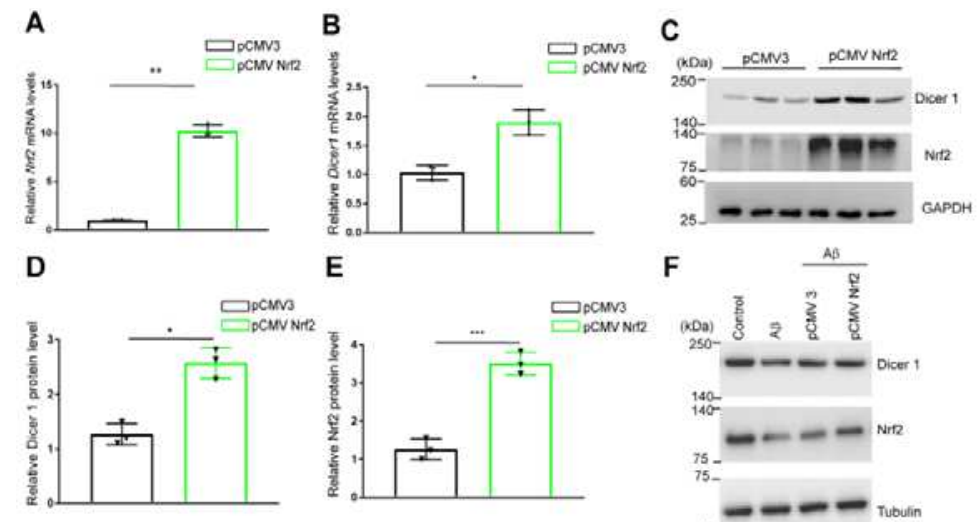

$F \quad \frac{A B}{\frac{9}{2}}$
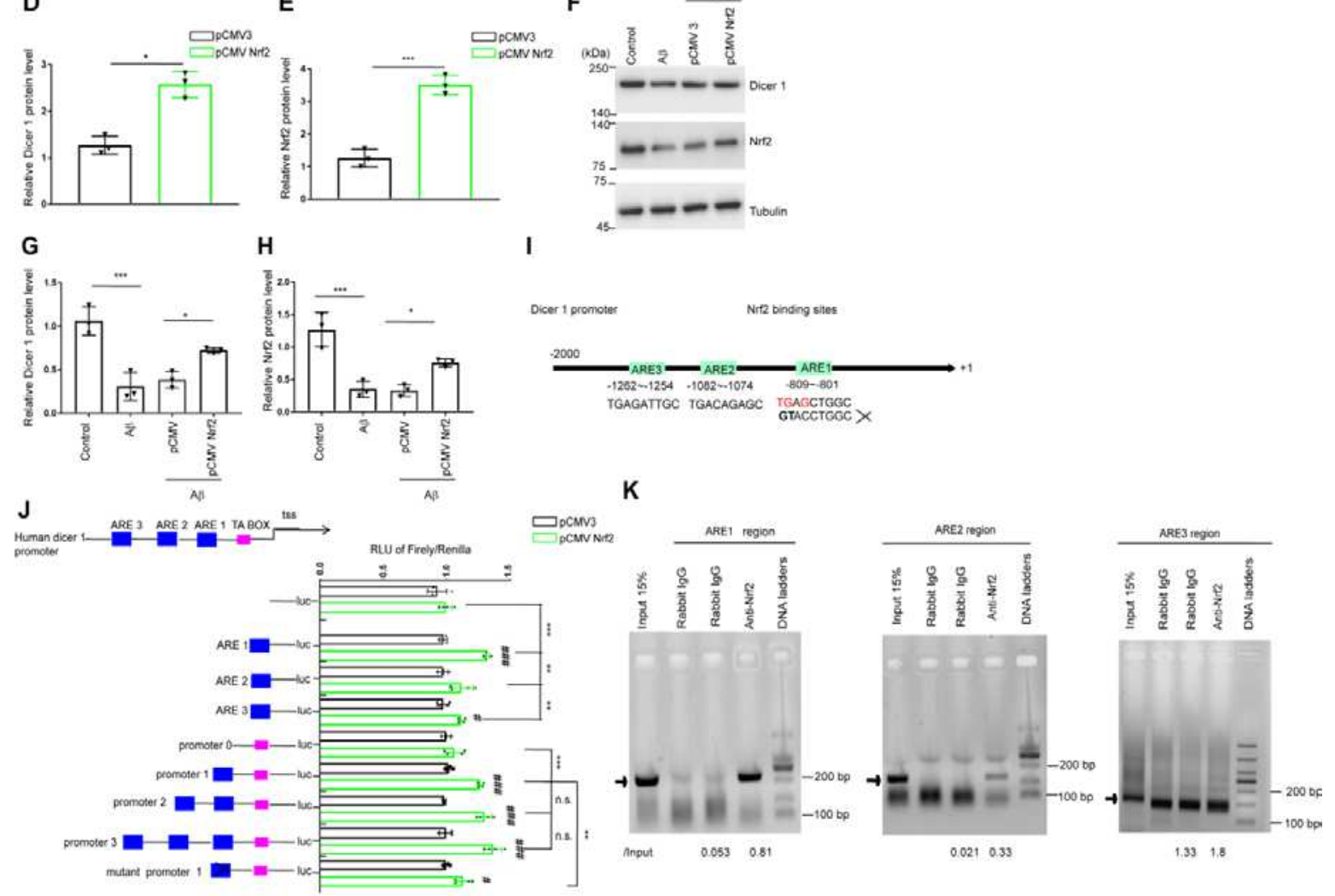

Figure 4. Nrf2 regulated Dicer1 production via Nrf2-ARE pathway. Neuro-2a cells were transfected with mouse Nrf2-expressing plasmids ( $\mathrm{pCMV} \mathrm{Nrf2,} 1.5 \mu \mathrm{g}$ ) or equal amount of vehicle plasmid (pCMV3) in 6-well plates. Examination of Nrf2 or Dicer1 mRNA was performed by qRT-PCR analysis. (A-B) Determination of Nrf2 and Dicer1 mRNA levels by overexpression of Nrf2 in Neuro-2A cells. Student's $t$-test was used to compare the differences. ${ }^{*} \mathrm{p}=0.0016$ for comparison of the levels of Dicer1 mRNA, ${ }^{*} \mathrm{p}=0.0482$ for comparison of the levels of Nrf2 mRNA. (C) Neuro-2a cells were transfected with a murine Nrf2-expressing (pCMV Nrf2) or vehicle plasmid (pCMV3) by use of lipofectamine 2000 for $48 \mathrm{~h}$. At the end of the culture, the cells were harvested 
for western blot against Nrf2 and Dicer1. GAPDH was used as a loading control. (D) The protein levels of Dicer1/ GAPDH in C were normalized to pCMV3 group and averaged from three independent culture preparation. Student's $t$-test was used to compare the differences, $p C M V 3$ vs. $p C M V 3$ Nrf2, ${ }^{*} p=0.0004$. (E) The protein levels of Nrf2/GAPDH in C were normalized to $\mathrm{pCMV3}$ group and averaged from three independent culture preparation. Student's $t$-test was used to compare the differences, pCMV3 vs. pCMV3 Nrf2, ${ }^{* * *} \mathrm{p}=0.024$. (F) Neuro-2a cells were subjected to treatment by vehicle, $A \beta 42$ oligomer (100 $\mathrm{nM}$ ) for $48 \mathrm{~h}$ and in parallel experiments, the cells were transfected with pCMV3 or pCMV3 Nrf2 for $48 \mathrm{~h}$ and then subjected to A 342 for $48 \mathrm{~h}$. The cells were harvested for determination of Dicer1 and Nrf2 and Tubulin was used as a loading control. (G) The protein levels of Dicer1/ Tubulin in F were normalized to pCMV3 group and averaged from three independent culture preparation. Student's $t$ test was used to compare the differences, pCMV3 vs. pCMV3 Nrf2, ${ }^{*} p=0.0004$. (H) The protein levels of Nrf2/Tubulin in F were normalized to pCMV3 group and averaged from three independent culture preparation. Student's $t$-test was used to compare the differences, pCMV3 vs. pCMV3 Nrf2, ${ }^{* * *} p=0.024$. (I) ARE motifs were found by use of the JASPAR database (http://jaspar.genereg.net/) within the Dicer1 promoter. (J) The upper panel showed scheme of Dicer 1 promoter containing three predicted ARE sites (blue square), TA box (pink red), tss indicating transcriptional start site (tss). HEK293 T cells were transfected with individual construct (150 ng), Renilla luciferase plasmid (7.5 ng), and PCMV3 or pCMV Nrf2 plasmid expressing human Nrf2 (150 ng). At the end of culture, the cells were harvested and subject to luciferase activity assay. The results were averaged from five independent culture preparation. One-way ANOVA followed 
by Tukey's post hoc test was used to compare the differences. Comparing cotransfection of pCMV3 Nrf2 with co-transfection of pCMV3 in individual construct, ${ }^{\# \#}$ $\mathrm{p}=0.000219$ in ARE1, ${ }^{\#}=0.043$ in ARE3, ${ }^{\# \#} \mathrm{p}=0.00047$ in promoter $1,{ }^{\# \#} \mathrm{p}=0.000135$ in promoter2, ${ }^{\# \#} \mathrm{p}=0.000114$ in promoter 3 , and ${ }^{\#} \mathrm{p}=0.048$ in mutant promoter 1 . Under the condition of co-transfection of pCMV3 Nrf2, ARE1 vs. pGL6-luc, ${ }^{* *} p=0.00041$; ARE1 vs. ARE2, ${ }^{* *} \mathrm{p}=0.000825 ;$ ARE1 vs. ARE3, ${ }^{* *} \mathrm{p}=0.0093$; promoter 1 vs. promoter 0 , ${ }^{* * *} \mathrm{p}=0.000361$; promoter 1 vs. mutant promoter $1,{ }^{* *} \mathrm{p}=0.009$, promoter 1 vs. promoter 2 , $p=0.194$ (n.s); promoter 1 vs. promoter $3, p=0.254$ (n.s). (K) CHIP assay of the interaction between Nrf2 and the ARE motif-containing Dicer1 promoter in SK-N-BE cells. Arrows indicated the captured fragments containing ARE-1 or ARE-2 motif. The optical densities of bands were measured by Image $\mathrm{J}$ and the ratios relative to positive input were shown below the individual bands, respectively. 
A

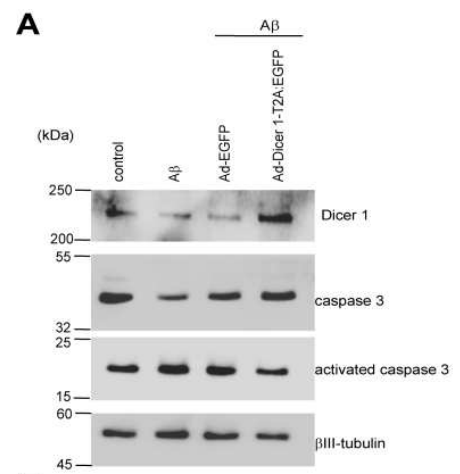

B

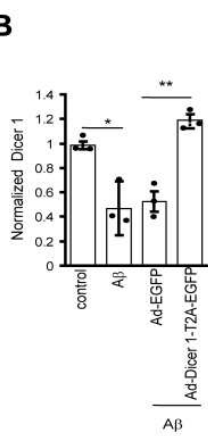

C

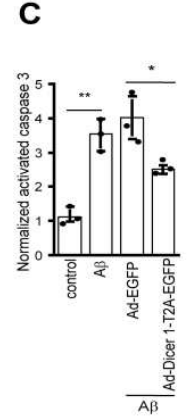

D

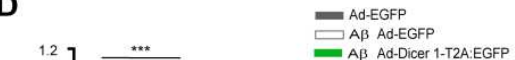

E
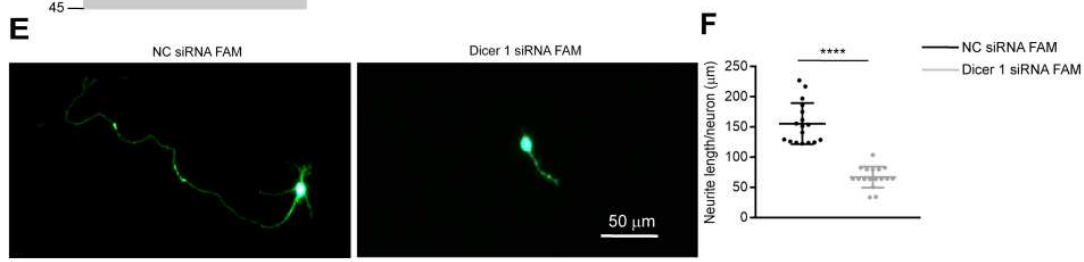

G
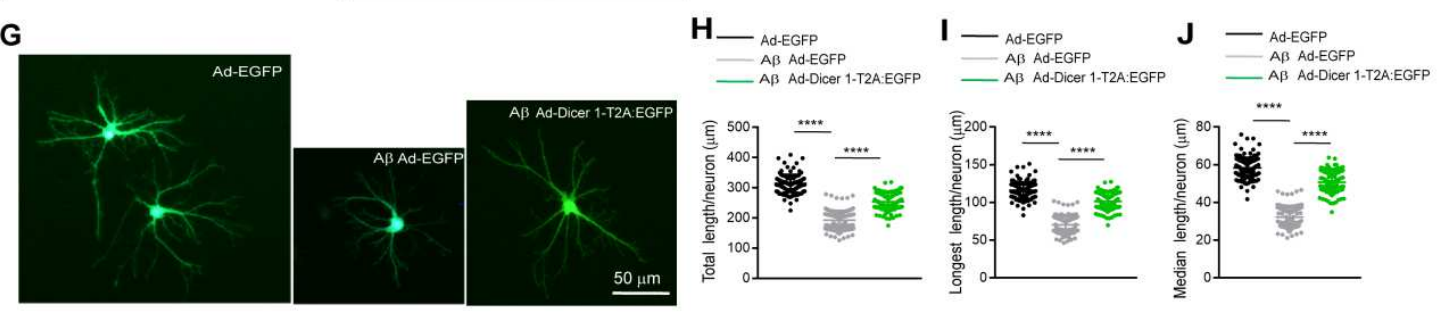

Figure 5. Overexpression of Dicer 1 reduced Aß42 oligomer-mediated apoptosis and neurite deficit in primary hippocampal neuronal cultures. (A) The primary murine hippocampal neurons at DIV 3 were treated with $A \beta 42$ oligomer (100 nM) for 24 h or subject to sham treatment in neurobasal medium plus $2 \%$ B27. In parallel experiments, the media were replaced with fresh neurobasal medium plus $2 \%$ B27 following $A \beta 42$ treatment, and then infected with vehicle virus, Ad-EGFP or Ad-dicer1T2A:EGFP virus $\left(5 \times 10^{7} \mathrm{vg} / \mathrm{mL}\right)$ for $48 \mathrm{~h}$. At the end of treatment, the cultures were harvested and homogenized for western blot against Dicer1, caspase 3, and activated caspase 3. $\beta$ III-tubulin was used as a loading control. (B) Dicer1 relative to $\beta$ III-tubulin were normalized to sham treatment and averaged from three independent culture preparation. One-way ANOVA followed by Tukey's post hoc test was used to compare the differences. Dicer1 was reduced by $A \beta 42$ oligomer treatment compared to sham 
treatment $\left({ }^{*} \mathrm{p}=0.033\right)$ and the reduction of Dicer 1 by $A \beta 42$ was rescued by infection of Ad-dicer1-T2A:EGFP compared to infection by vehicle virus $\left({ }^{* *} \mathrm{p}=0.0074\right)$. (C) Activated caspase3 relative to $\beta$ III-tubulin in A were normalized to sham treatment and averaged from three independent culture preparation. One-way ANOVA followed by Tukey's post hoc test was used to compare the differences. Activated caspase3 was increased by $A \beta 42$ oligomer compared to sham treatment $\left({ }^{* *} \mathrm{p}=0.0061\right)$, which was reduced by infection of Ad-dicer1-T2A:EGFP compared to infection by vehicle virus ( $\left.{ }^{*} \mathrm{p}=0.023\right)$. (D) The loss of cell viability of hippocampal neurons treated by $A \beta\left({ }^{* \star *} p=0.00067\right)$ was significantly rescued by infection with Ad-dicer1-T2A:EGFP compared to infection by vehicle virus $\left({ }^{*} \mathrm{p}=0.047\right)$. The values were averaged from quadruplicate cultures with three independent culture preparation. (E) Knockdown of Dicer1 reduced neurite length in primary cortical neuronal cultures. $7 \times 10^{4}$ cortical neurons were transfected with 6 fluorescein amidite (FAM)-labeling Dicer1 siRNA duplex (Dicer1 siRNA FAM) or scrambled siRNA duplex (NC siRNA FAM) for $12 \mathrm{~h}$, respectively. Neurons were observed with fluorescence microscope. The images were representative images of neurons transfected with Dicer1 siRNA FAM or NC siRNA FAM. Bar, $50 \mu \mathrm{m}$. (F) Total neurite length per cortical neuron averaged from 17 neurons under either transfection condition from E. Student's $t$-test was used to compare the difference, ${ }^{\star * \star *} p<0.0001$. (G) Representative hippocampal neurons (150 neurons for each type of treatment) infected by Ad-EGFP virus (Ad-EGFP), A 342 treatment followed by infection with AdEGFP virus ( $A \beta$ Ad-EGFP) and $A \beta 42$ treatment followed by infection with Ad-Dicer1T2A:EGFP virus (Aß Ad-Dicer1:T2A:EGFP) were indicated. (H) Total neurite length, (I) the longest length and $(\mathbf{J})$ median length of hippocampal neurons with treatment from $G$ 
were calculated and averaged. $A \beta 42$ treatment reduced neurite length compared to sham treatment which was rescued by infection with Ad-dicer 1-T2A:EGFP virus.

${ }^{* * *} p<0.0001$, The neurite length averaged from 150 neurons in each treatment and One-way ANOVA followed by Tukey's post hoc test was used to compare the differences. 
A

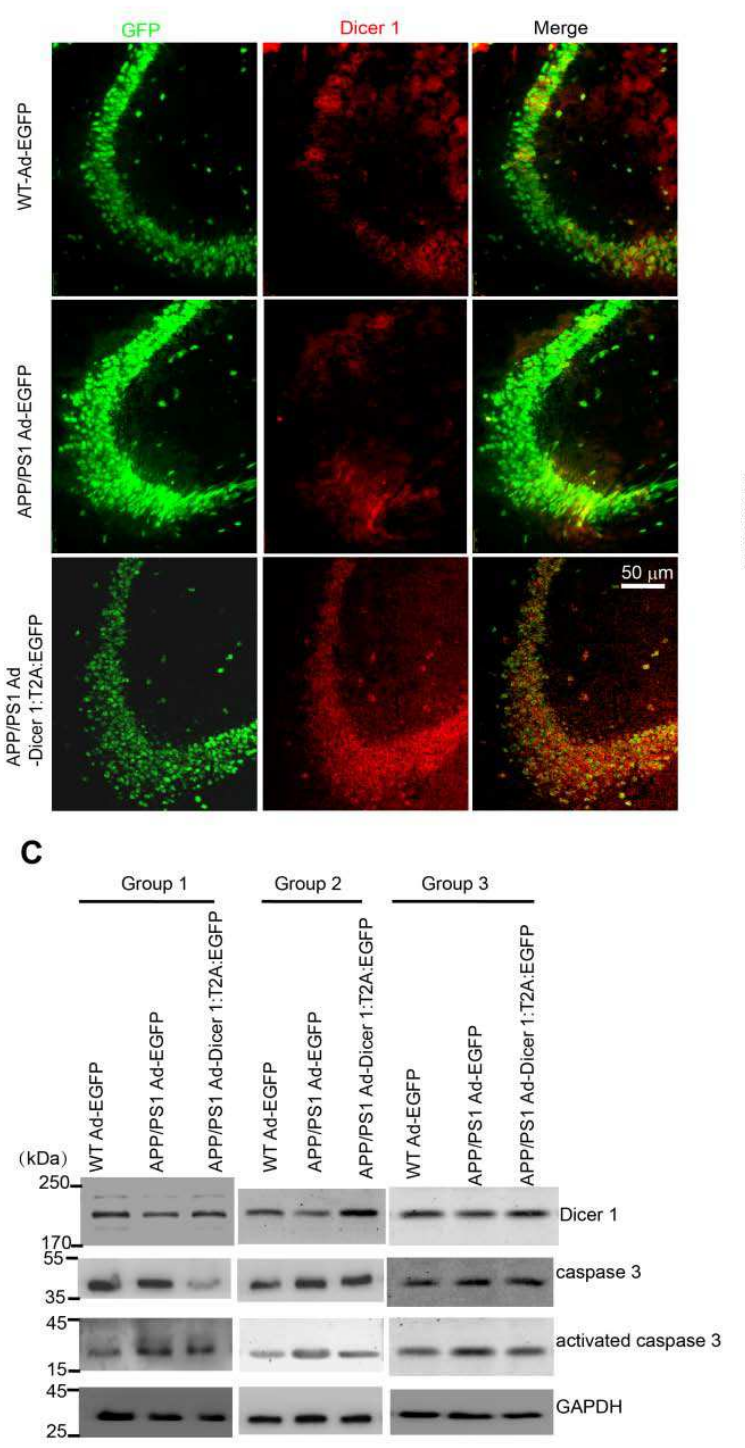

B
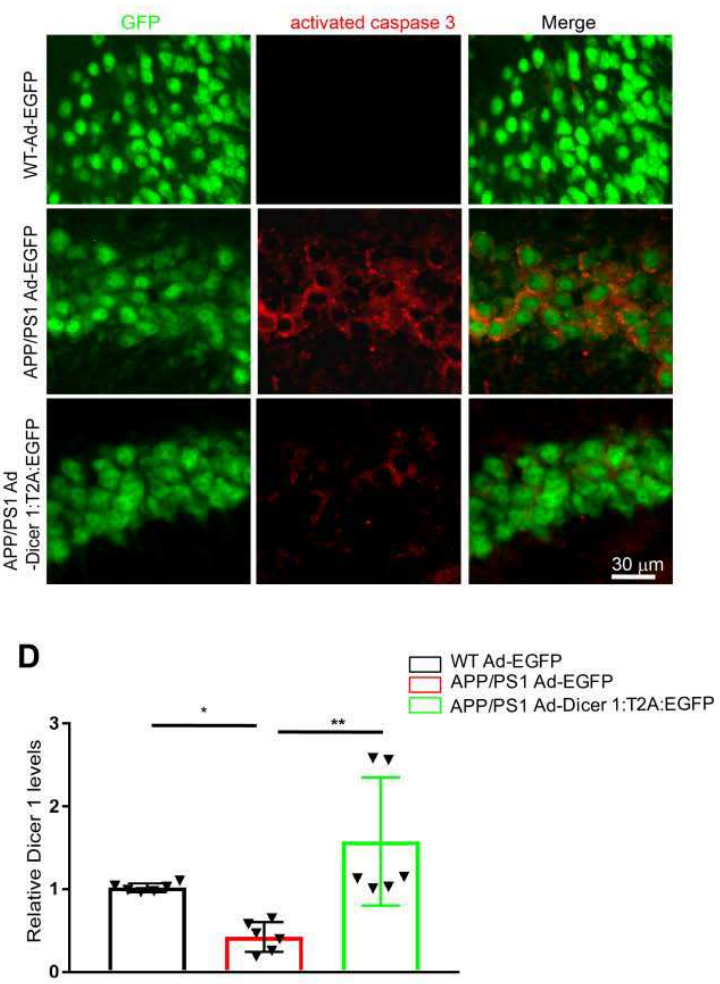

$\mathbf{E}$

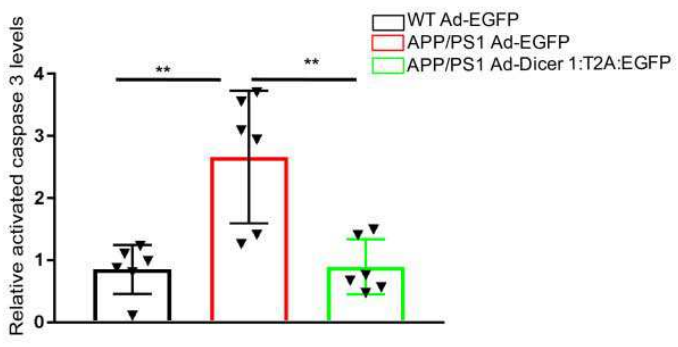

Figure 6. Overexpression of Dicer1 in APP/PS1hippocampus inhibited activated caspase 3. APP/PS1 mice or WT littermate at the age of 3.5-/4-month were injected with Ad-EGFP or Ad-Dicer1-T2A:EGFP virus $\left(1.2 \times 10^{9} \mathrm{vg} / \mathrm{mL}\right)$ in CA3 hippocampi. 17 days after injection, the injected mice were perfused and each brain was sagittally cut into $\sim 60$ sections at $10 \mu \mathrm{m}$-thick for ach section. The sections every other six sections were chosen for double staining of Dicer1 (red) (A) and activated caspase 3 (red) (B). The typical images of CA3 hippocampal region were indicated. (A) Dicer1 staining (red) 
in APP/PS1 CA3 hippocampus was reduced compared to those in WT littermate and infection with Ad-Dicer 1-T2A:EGFP virus dramatically increased Dicer1 staining in CA3 hippocampus. The images were typical of 60 sections from six mice. Bar, $50 \mu \mathrm{m}$. (B) The staining of activated caspase 3 (red) in APP/PS1mice was increased compared to those in WT and this increase in staining was mitigated by infection with Ad-Dicer1T2A:EGFP virus. The images were typical of 60 sections from six mice. Bar, $30 \mu \mathrm{m}$. (C) The CA3 hippocampi were harvested, homogenized, and subjected to centrifugation at $12,000 \mathrm{Xg}$, and the supernatants were used for western blot against Dicer1, caspase3, and activated caspase3, and typical blots were indicated. (D) Dicer1 was reduced in APP/PS1 hippocampi compared to WT ( $\left.{ }^{*} \mathrm{p}=0.0217\right)$, which was increased by infection with Ad-Dicer1-T2A:EGFP virus $\left({ }^{* *} \mathrm{p}=0.0093\right)$. The optical densities averaged from six mice in each group and Kruskal-Wallis test was used to compare the differences. (E) Activated caspase 3 relative to GAPDH was increased in APP/PS1 hippocampi compared to WT $\left({ }^{* *} p=0.00681\right)$, which was reduced by infection with AdDicer1-T2A:EGFP virus $\left({ }^{* *} p=0.0067\right)$. The optical densities averaged from six mice in each group and Kruskal-Wallis test was used to compare the differences. 

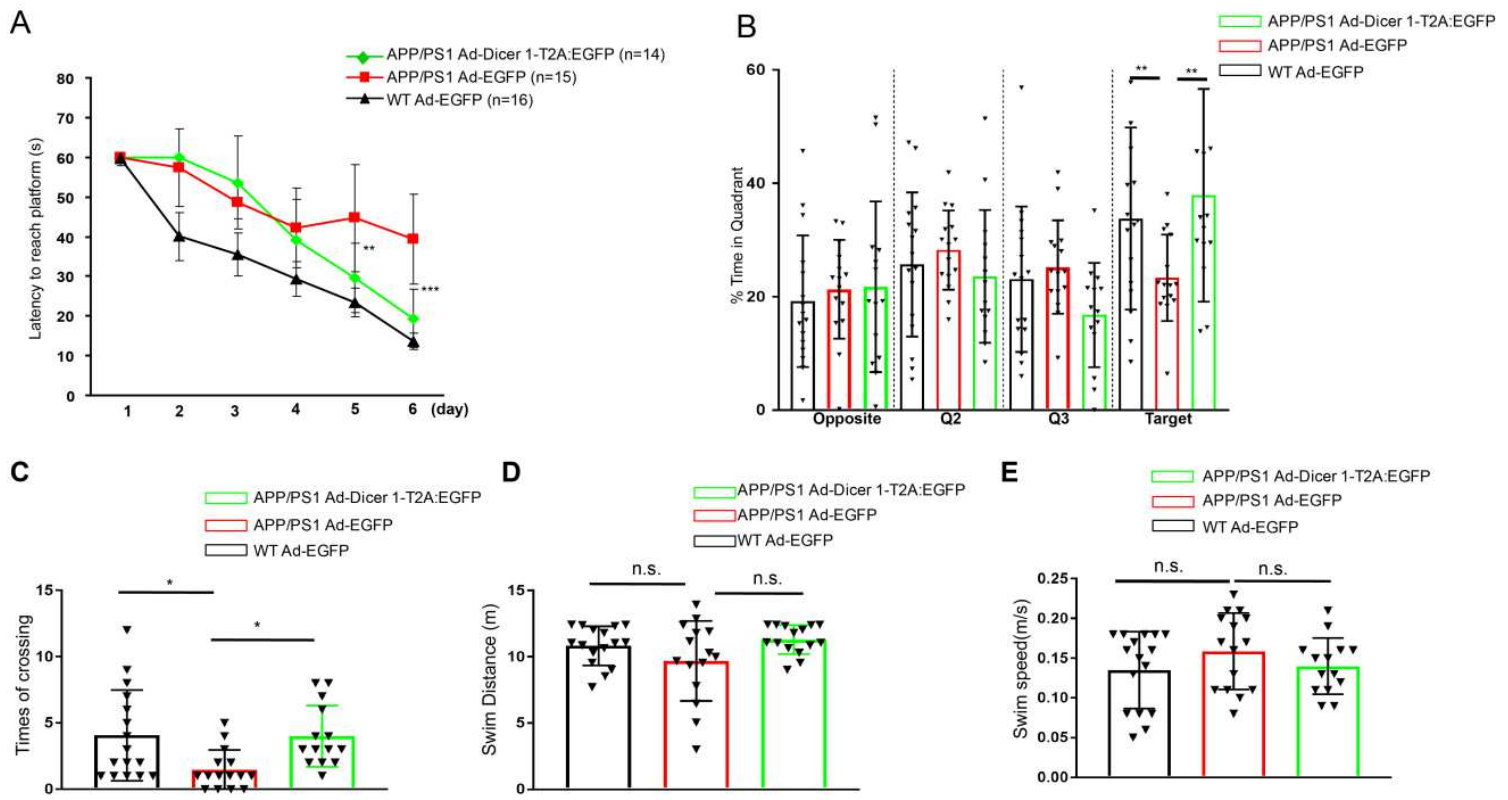

Figure 7. Overexpression of Dicer 1 in CA3 hippocampus significantly improved spatial learning in APP/PS1 mice. Ad-Dicer1-T2A:EGFP virus $\left(1.2 \times 10^{9} \mathrm{vg} / \mathrm{mL}\right)$ or equal amount of Ad-EGFP virus were injected into CA3 hippocampus in APP/PS1or WT mice, at the age of 3.5-4 months, and behavior test was conducted at 17-23 days after injection. (A) Learning curve of the mice from WT mice injected with Ad-EGFP virus $(n=16)$, APP/PS1 mice injected with Ad-EGFP virus $(n=15)$ or Ad-Dicer1-T2A:EGFP virus $(n=14)$. The differences of latency to reach platform were analyzed by use of twoway ANOVA with repeated measures against day and treatment (virus injected), $p_{\text {day }}$ $=0.00001, p_{\text {treatment }}=0.0241, p_{\text {interaction }}=0.0148$. Tukey's post hoc test was further used to compare the differences between APP/PS1 mice injected with Ad-Dicer1-T2A:EGFP virus and APP/PS1mice injected with Ad-EGFP virus, ${ }^{* *} \mathrm{p}=0.008$ on day 5 and ${ }^{\star * *} p=0.00001$ on day 6 . (B) Analysis of probe trial indicated that preference of APP/PS1mice for the target quadrant was enhanced by Ad-Dicer 1-T2A:EGFP virus injection in CA3 hippocampi. Two-way ANOVA with repeated measures against 
quadrant and treatment was used to compare the differences, $p_{\text {quadrant }}=0.1122, p_{\text {type }}=1$, $p_{\text {interaction }}=0.0046$. Tukey's post hoc test was further used to compare the differences among groups, APP/PS1 Ad-Dicer 1-T2A:EGFP $(n=16)$ vs. APP/PS1 Ad-EGFP( $n=15)$, ${ }^{* *} \mathrm{p}=0.0087$; APP/PS1 Ad-EGFP ( $\left.\mathrm{n}=15\right)$ vs. WT Ad-EGFP $(\mathrm{n}=16),{ }^{* *} \mathrm{p}=0.0023$. (C) Frequencies of crossing target platform was increased by Dicer1 overexpression. Tukey's post hoc test was further used to compare the differences among groups, APP/PS1 Ad-Dicer 1-T2A:EGFP ( $\mathrm{n}=14)$ vs. APP/PS1 Ad-EGFP ( $\mathrm{n}=15)$; ${ }^{*} \mathrm{p}=0.043$; APP/PS1 Ad-EGFP ( $n=15)$ vs. WT Ad-EGFP ( $n=16),{ }^{*} p=0.039$. (D) Swim distance and (E) Swim speed did not show differences among groups (n.s). 


\section{Supplemental Figure legends}

A
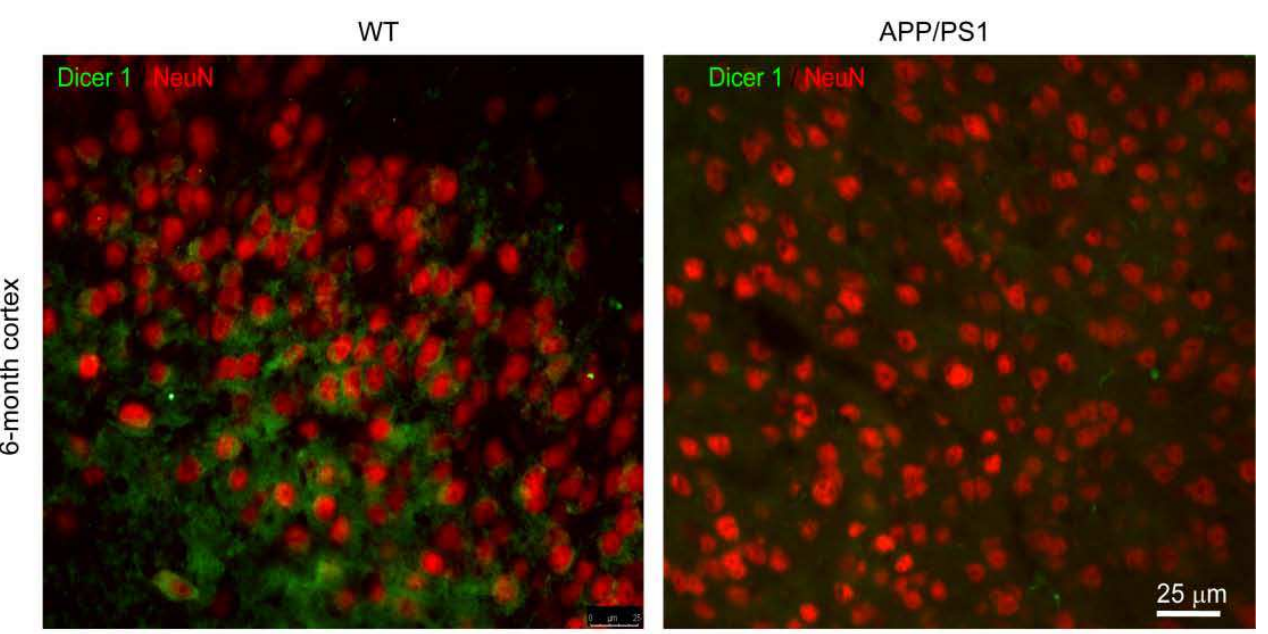

B
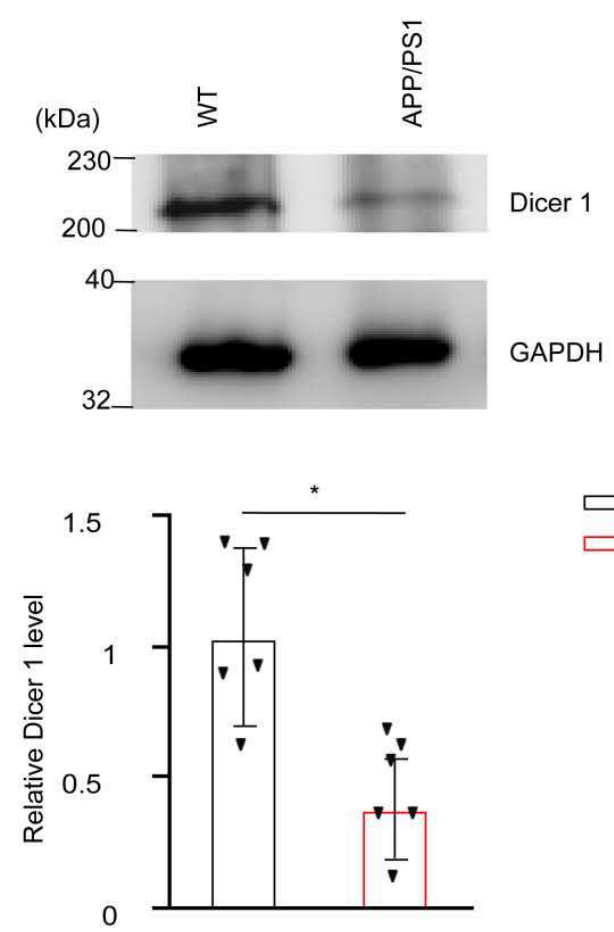

$\square$ WT

$\square$ APP/PS1

Figure S1. Reduction of Dicer 1 in 6-month-old APP/PS1 cortex. (A) Representative image of Dicer1 staining in parietal cortex with double staining by anti-Dicer1(green) and anti-NeuN(red). ( $n=3$, per genotype). Bar, $25 \mu \mathrm{m}$ for all panels. (B) The homogenates of parietal lobe from WT $(n=6)$ and APP/PS1 $(n=6)$ were subject to 
western blot against Dicer1. GAPDH was used as a loading control. The optical densities of Dicer1 relative to GAPDH averaged from three independent experiments. Mann-Whitney U test was used to compare the differences, ${ }^{*} \mathrm{p}=0.022$. 


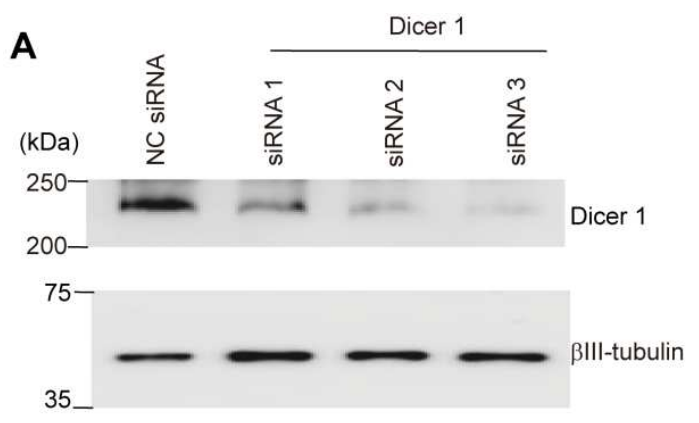

B

C

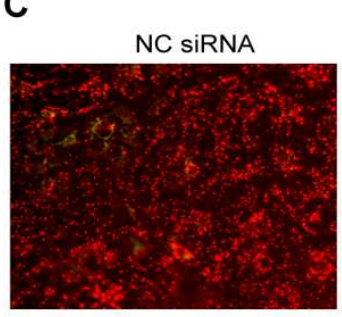

Dicer 1 siRNA

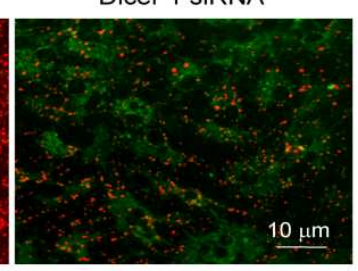

E

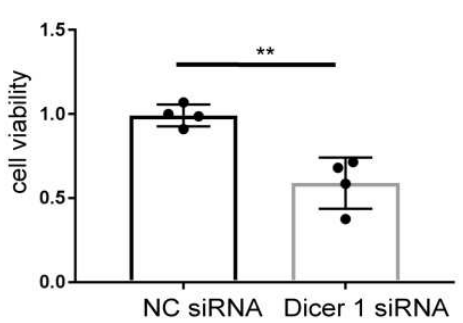

F
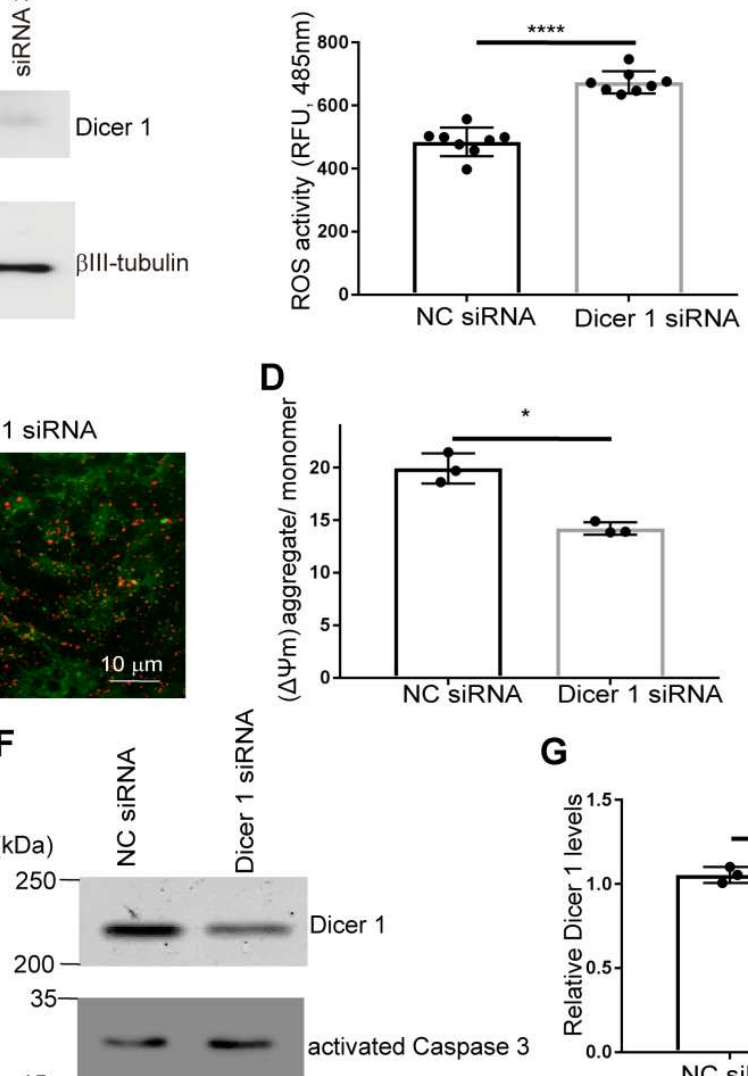

D

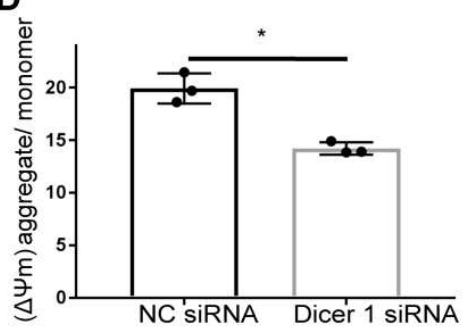

G
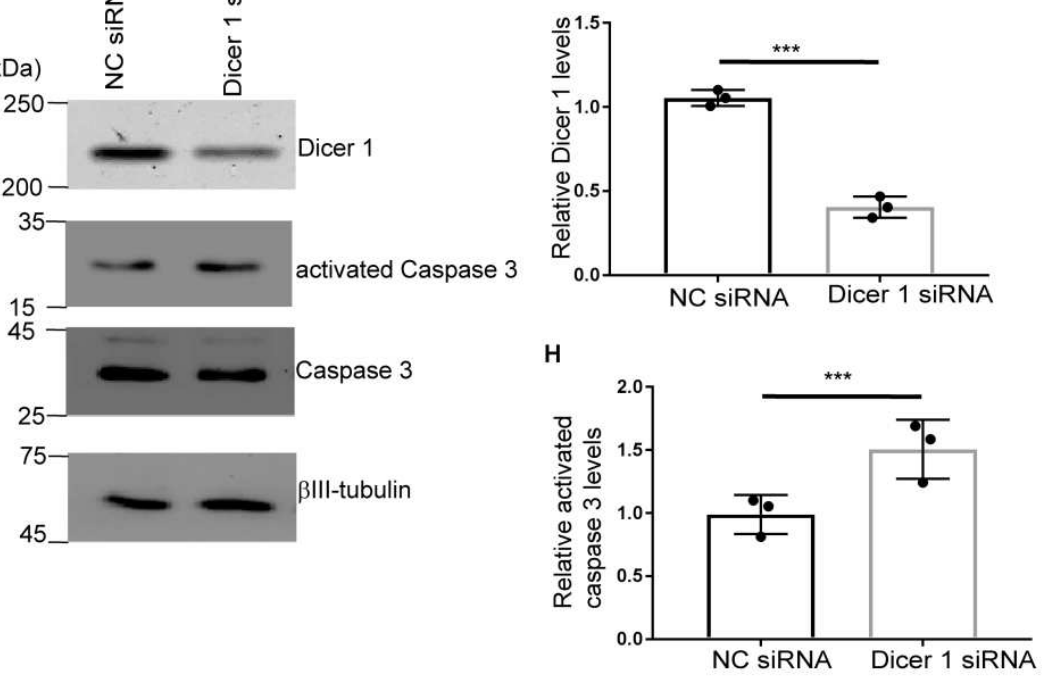

Figure S2. Knocking down Dicer 1 induced production of reactive oxygen species (ROS), reduced mitochondrial membrane potential, enhanced apoptosis in primary murine cortical neuronal cultures. (A) The cortical neuronal cultures were subjected to transfection with Dicer1 siRNA1-3 (50 pM for each) or equal mass of scrambled siRNA (NC siRNA), respectively. After $48 \mathrm{~h}$, the cells were harvested for immunoblotting against Dicer1. The experiments were repeated twice and the siRNA3 
was chosen as Dicer1 specific siRNA. (B) The ROS levels in cortical neuronal cultures ( CNs) were measured by incubating 2',7'-dichlorodihydrofluorescein diacetate for 20 $\min$ at $37^{\circ} \mathrm{C}$. The fluorescence values in relative fluorescence units (RFU) were acquired in a plate reader at $485 \mathrm{~nm}$ and were averaged from four independent culture preparation with duplicate cultures in each preparation. ${ }^{* * *} \mathrm{p}=0.00001$. (C) Representative images of JC-1 staining in CNs subject to Dicer1 knockdown as above. Red fluorescence represented as red-J aggregate in mitochondria at hyperpolarized membrane potentials under healthy condition whereas green fluorescence as monomer in cytoplasm at depolarized membrane potentials under the context of compromised mitochondria. Bar $10 \mu \mathrm{m}$ for all panels. (D) Mitochondrial membrane potential $\left(\Delta \Psi_{\mathrm{m}}\right)$ of CNs subject to Dicer1 knockdown. The neurons were subject to Dicer1 knockdown and mitochondria $(30 \mu \mathrm{g})$ were isolated and stained with $\mathrm{JC}-1$. The values of mitochondrial membrane potential $(\triangle \Psi \mathrm{m})$ were indicated by the ratios between aggregated/monomer RFU and averaged from three independent culture preparations. Student's $t$-test was used to compare the differences, ${ }^{*} p=0.0369$. (E) Viability of neurons subjected to Dicer1 knockdown. CNs were transfected with Dicer 1 siRNA or NC siRNA as described in the materials and methods. The absorption values at OD 450nm in Dicer1 siRNA group were measured and normalized to NC siRNA group. Student's $t$-test was used to compare the differences, ${ }^{* *} \mathrm{p}=0.0028$ for comparison. (F) CNs were transfected with Dicer1 siRNA (50 pM) or control siRNA duplex (50 pM) per well and subjected to western blot examination of Dicer1, activated caspase3, caspase 3 and $\beta$ III-tubulin was used as a loading control. (G) The effects of knockdown by Dicer1 siRNA were normalized to those of NC siRNA and averaged from three 
independent culture preparation. Student's t-test was used to compare the differences, ${ }^{* * *} \mathrm{p}=0.0025$ for comparison. (G) The optical densities of activated caspase- 3 relative to BIII-tubulin from $\mathrm{F}$ were normalized to NC siRNA group, and averaged from three independent culture preparation. Student's $t$-test was used to compare the differences, ${ }^{* * *} \mathrm{p}=0.008$ for comparison. 
A

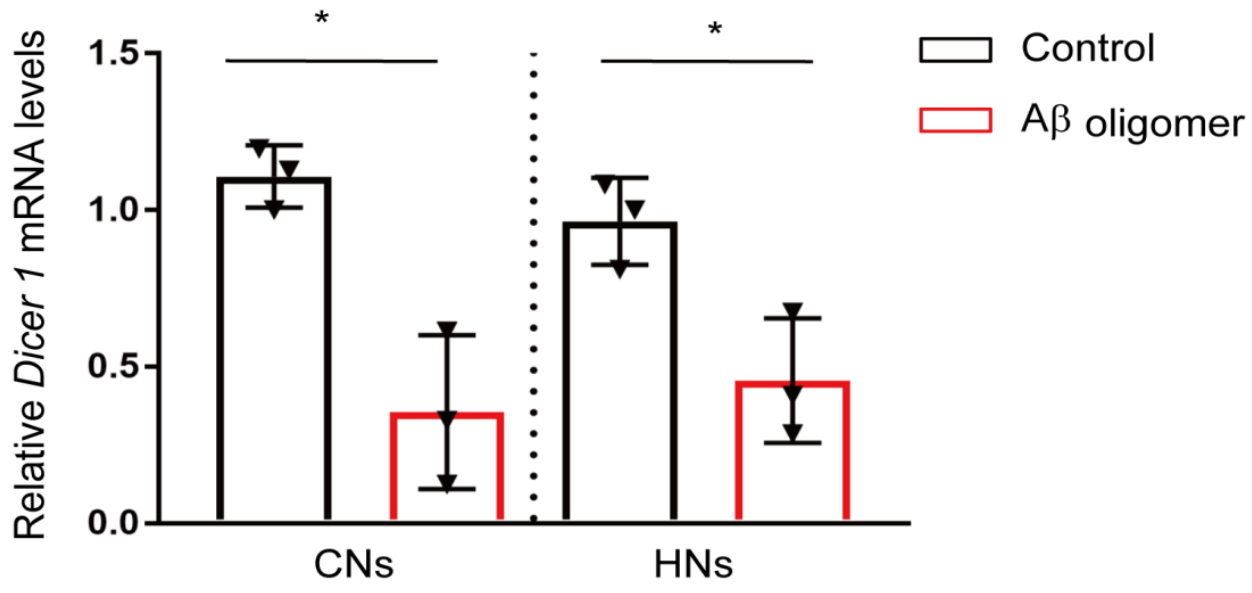

B

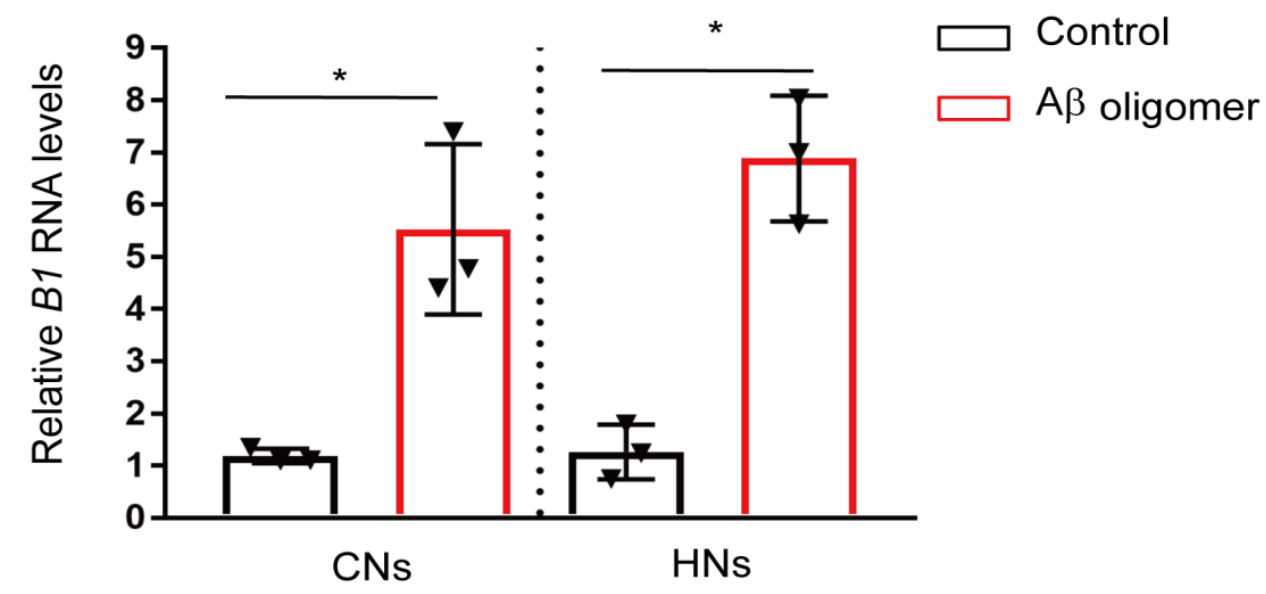

Figure S3. A 42 oligomer decreased Dicer1 mRNA and increased B1 RNA in primary neuronal cultures. Primary murine cortical neuronal cultures were treated with $\mathrm{A} \beta 42$ oligomer (100 $\mathrm{nM}$ ) for $48 \mathrm{~h}$ and subject to real-time quantitative PCR examination of Dicer1 mRNA (A) and B1 RNA (B). Student's t-test was used to compare the differences. ${ }^{*} \mathrm{p}=0.0277$ for comparison of the levels of Dicer 1 mRNA in CNs, ${ }^{*} p=0.0387$ for comparison of Dicer1 RNA in HNs in $A .{ }^{*} p=0.0469$ for comparison of the levels of $B 1$ RNA in CNs, ${ }^{*} p=0.0252$ for comparison of B1 RNA in HNs. The results were averaged from three independent culture preparation. 

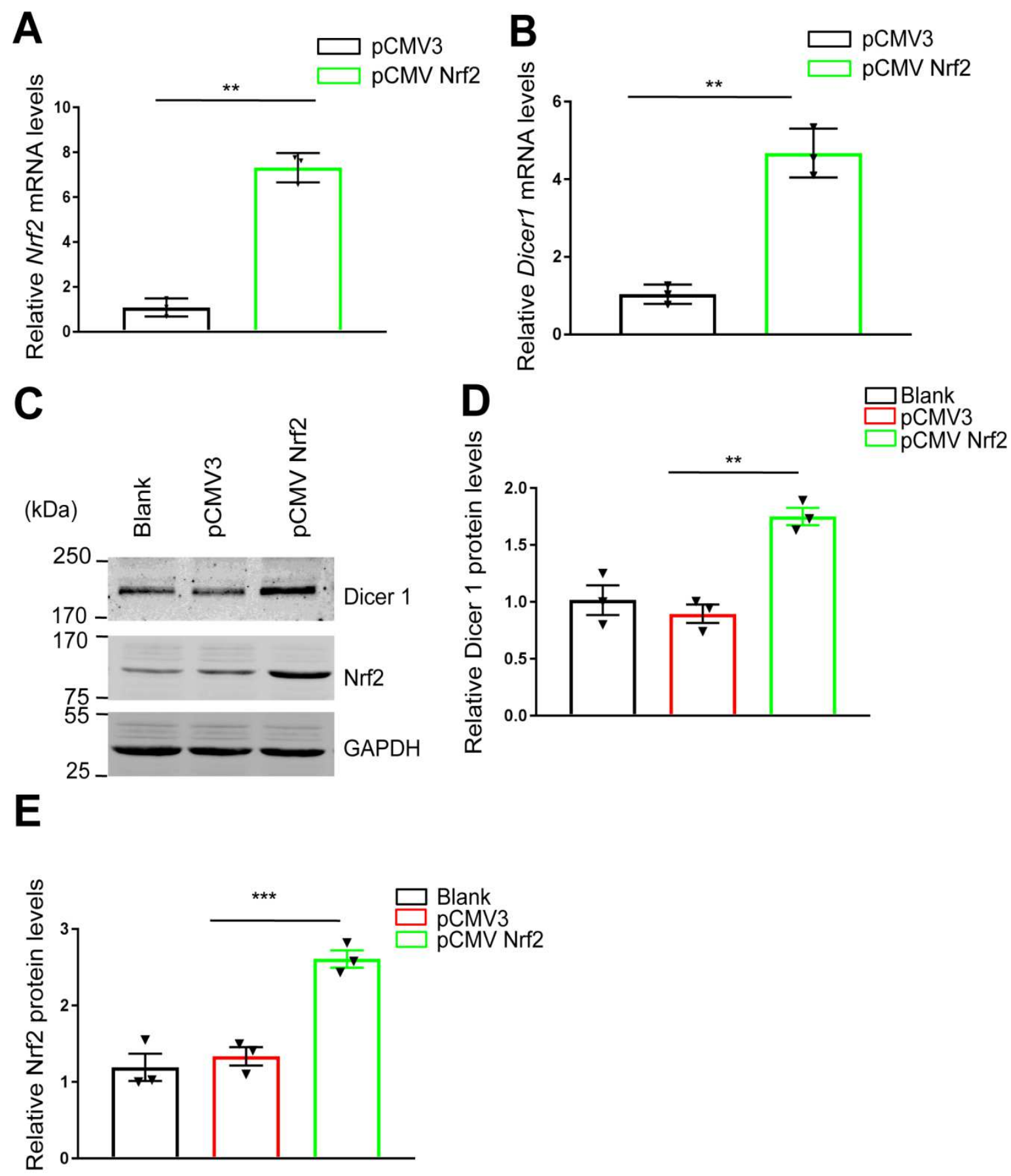

Figure S4. Enhancing expression of Nrf2 increased expression of Dicer1 in SK-NBE cells. (A-B) SK-N-BE cells were left untreated (Blank) or transfected with human Nrf2-expressing plasmids (pCMV Nrf2, $1.5 \mu \mathrm{g}$ ) or equal amount of vehicle plasmid 
(pCMV3) in 6-well plates. Examination of Nrf2 or Dicer1 mRNA was performed by qRTPCR analysis. Determination of Nrf2 or Dicer1 mRNA levels by overexpression of Nrf2 in SK-N-BE cells. Student's $t$-test was used to compare the differences. ${ }^{*} \mathrm{p}=0.0038$ for comparison of the levels of Dicer $1 \mathrm{mRNA},{ }^{*} \mathrm{p}=0.0014$ for comparison of the levels of Nrf2 mRNA. (C) Forty-eight hours after transfection, the cells were harvested for western blot against Nrf2 and Dicer1. GAPDH was used as a loading control. (D) The protein levels of Dicer1 relative to GAPDH in C were normalized to pCMV group and averaged from three independent culture preparation. One-way ANOVA followed by Tukey's post hoc test was used to compare the differences, pCMV3 vs. pCMV3 Nrf2, ${ }^{* *} p=0.0021$. (E) The protein levels of Nrf2/ GAPDH in $C$ were normalized to $p C M V$ group and averaged from three independent culture preparations. One-way ANOVA, followed by Tukey's post hoc test was used to compare the difference, pCMV3 vs. pCMV3 Nrf2, ${ }^{* *} \mathrm{p}=0.0017$. 
DAPI
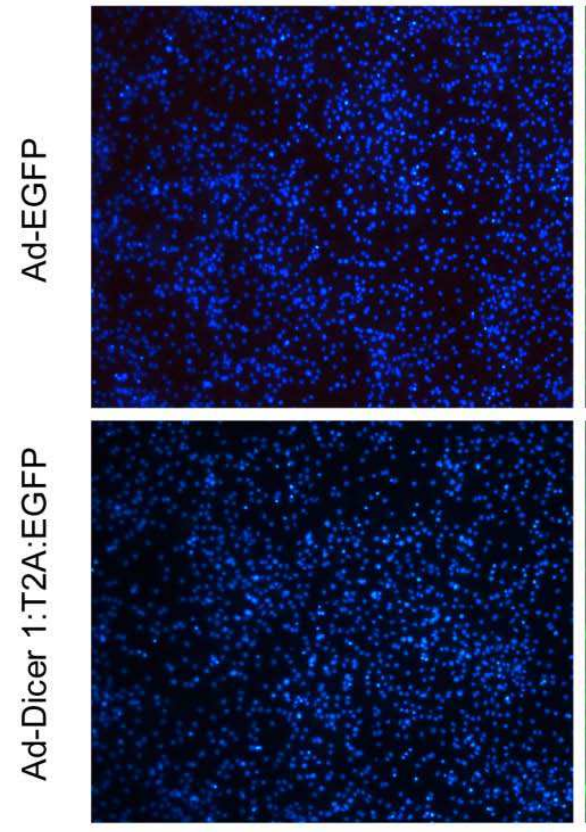

EGFP
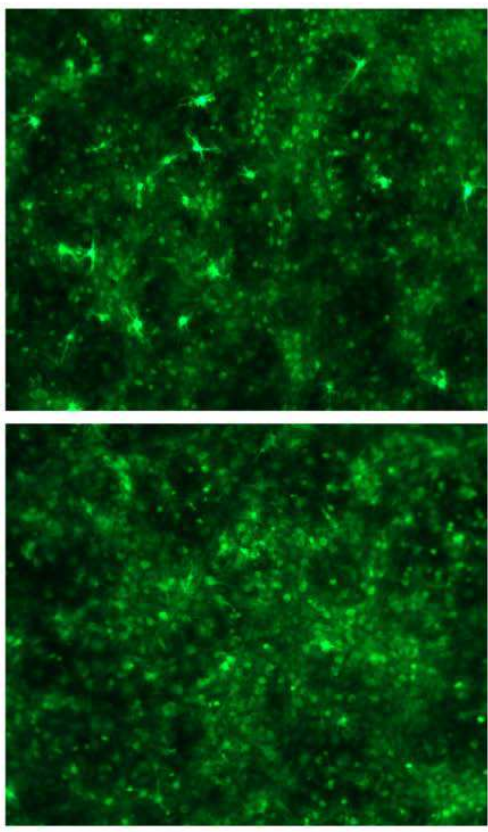

Merge
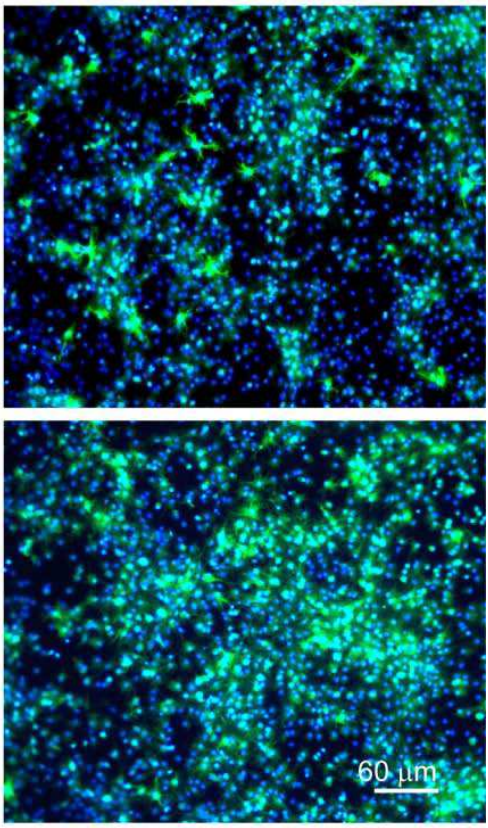

Figure S5. Infection of hippocampal neuronal cultures by adenovirus. The primary mouse hippocampal neurons were culture as described in the materials and methods. At DIV 3, the cells were infected with Ad-pCMV-EGFP or Ad-pCMV-

Dicer1:T2A:EGFP $\left(5 \times 10^{7} \mathrm{vg} / \mathrm{mL}\right)$ for $60 \mathrm{~h}$. The images were randomly selected and taken under fluorescence microscope with fluorophore filter for DAPI or GFP. Scale bar, $60 \mu \mathrm{m}$. 


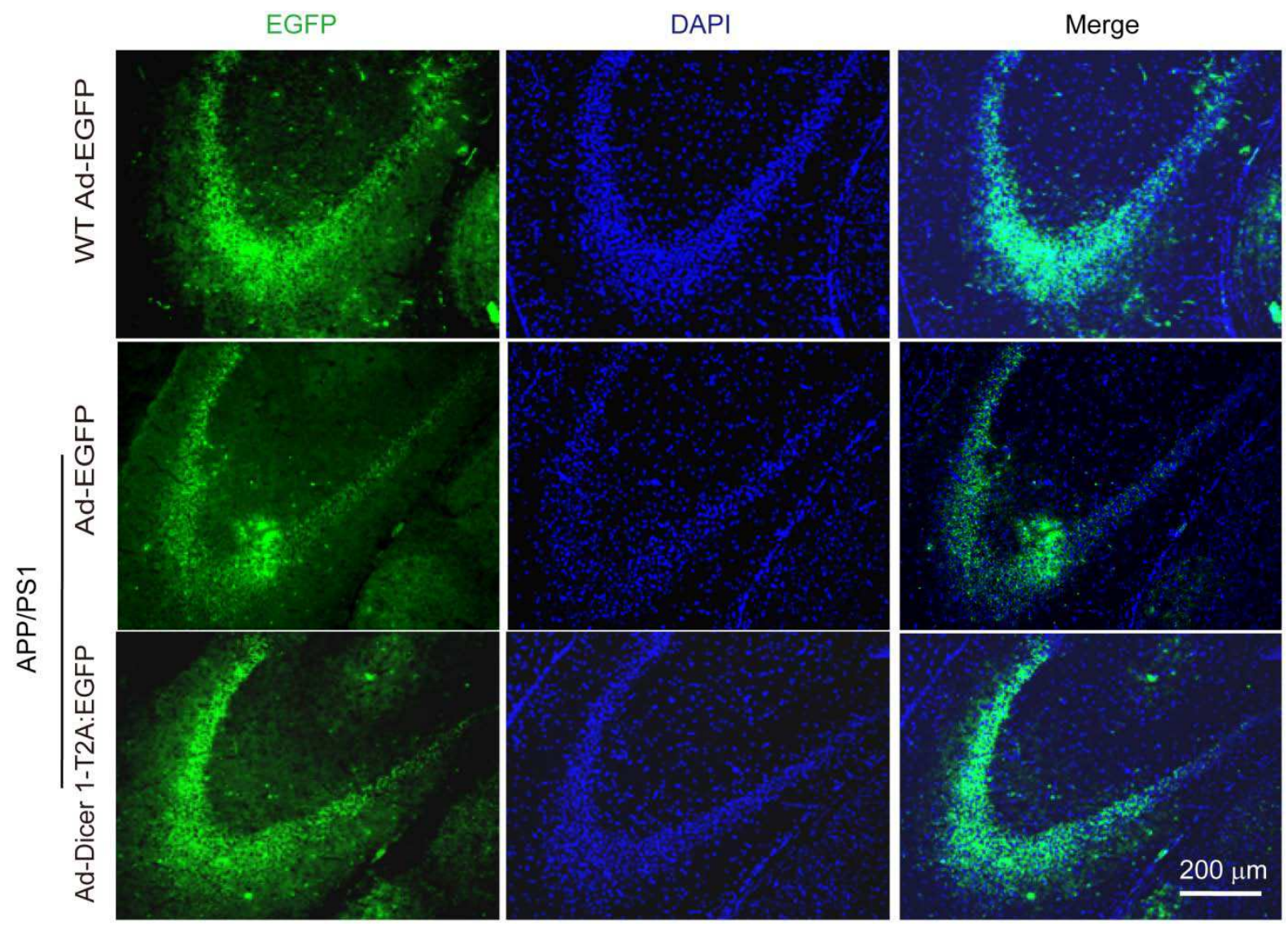

Figure S6. Infection of CA3 region by intrahippocampal injection of virus. WT or APP/PS1 mice at 4-month were injected with $2 \mu \mathrm{L}$ of Ad-pCMV-EGFP or Ad-pCMVDicer1:T2A:EGFP virus $\left(1.2 \times 10^{9} \mathrm{vg} / \mathrm{mL}\right)$ in each hemisphere as described in the material and methods. After 23 days, the mice were subjected to decapitation, the brain was processed into $10 \mu \mathrm{m}$ sections, fixed in $4 \%$ paraformaldehyde and stained for DAPI. The images were captured with fluorescence microscope with different fluorophore filter for DAPI and GFP. Scale bar, $80 \mu \mathrm{m}$. 
A
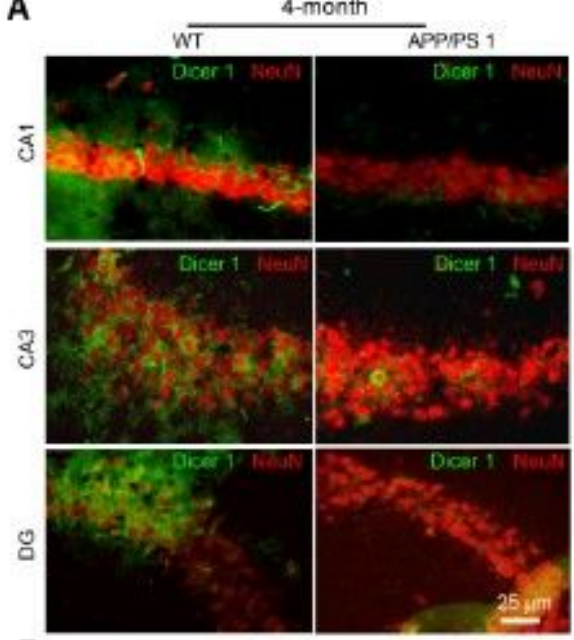

B
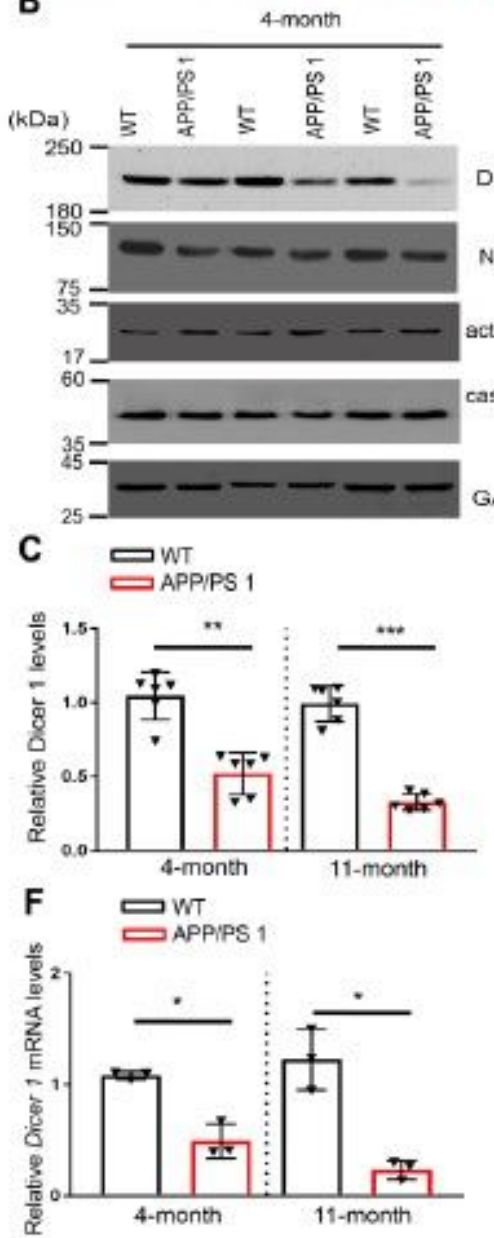

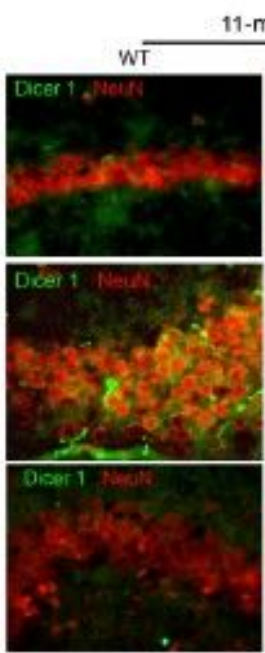

11-month

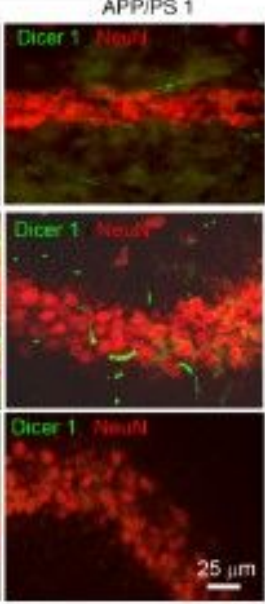

25 um

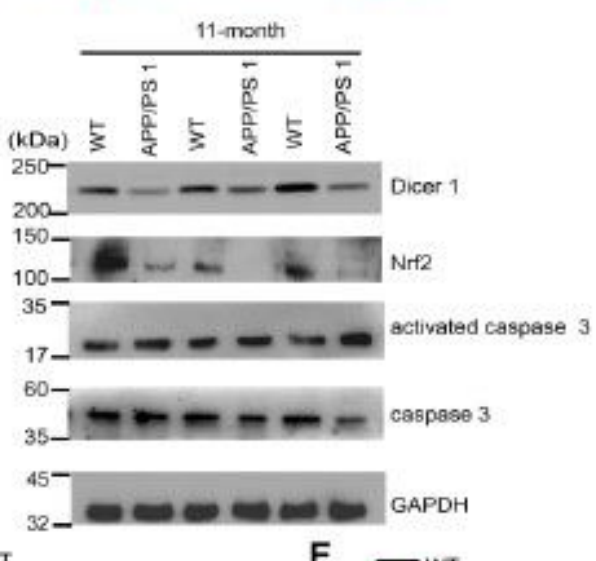

D

E 口wT

DAPP,PS 1

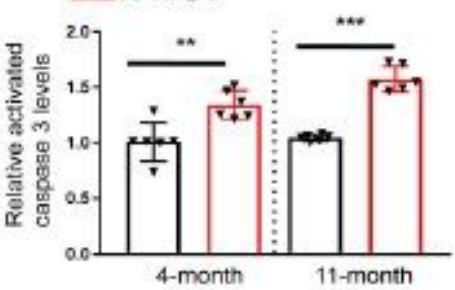

G 口WT

שAPPIPS 1

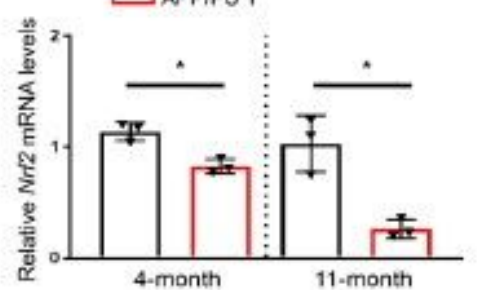

\section{Figure 1}

Reduction of Dicer 1, decreased Nrf2 and increased activated caspase-3 in the hippocampus of APP/PS1 mice. (A) APP/PS1 brains were processed into sections which were doubly stained for Dicer1 (green) or NeuN (red). The images were typical staining of CA1, CA3, or DG ( $n=3$ mice per genotype). Bar, $25 \mu \mathrm{m}$ for 
all panels. (B) The supernatants from homogenized hippocampai at the age of 4-/11 months were collected and subject to examination of Dicer1, Nrf2 , caspase-3, and activated caspase-3 by western blot. GAPDH was used as a loading control. The protein levels of Dicer1 and Nrf2 in the hippocampal tissues were reduced in APP/PS1 mice ( $n=6,3$ male and 3 female) compared to WT littermate $(n=6,3$ male and 3 female), respectively, at the age of 4 months (Left panel) or at the age of 11 months (right panel). By contrast, the protein levels of activated caspase-3 were increased in APP/PS1 mice $(n=6)$ compared to WT $(n=6)$ at the age of 4-/11 months. (C) The optical densities of Dicer1 relative to GAPDH were normalized to WT at the age of 4 months ( $\left.{ }^{*} p=0.0092\right)$ or 11 months $(* \star \star p=0.0002)(n=6$ per genotype, Mann-Whitney U test). (D) Nrf2 relative to GAPDH were normalized to WT (Mann-Whitney U test, ${ }^{* \star *} \mathrm{p}=0.0001$ in 4-month, ${ }^{* *} \mathrm{p}=0.0038$ in 11-month, $\mathrm{n}=6$ per genotype). (E) Activated caspase 3 relative to GAPDH were normalized to WT (Mann-Whitney $U$ test, ${ }^{*} \mathrm{p}=0.0098$ in 4-month, ${ }^{\star * \star} \mathrm{p}=0.0008$ in 11-month, $n=6$ per genotype), respectively. (F) Dicer1 mRNA was examined in WT $(n=3)$ and in APP/PS1 mice $(n=3$ ) at the age of 4 months ( $p=0.0335)$ or 11 months ( $p=0.0304$ ) (paired t-test). (G) Nrf2 mRNA was examine in WT $(n=3)$ and in APP/PS1 mice $(n=3)$ at the age of 4 months $(p=0.0167)$ or 11 months $(p=0.0195)$ (paired t-test). 
A
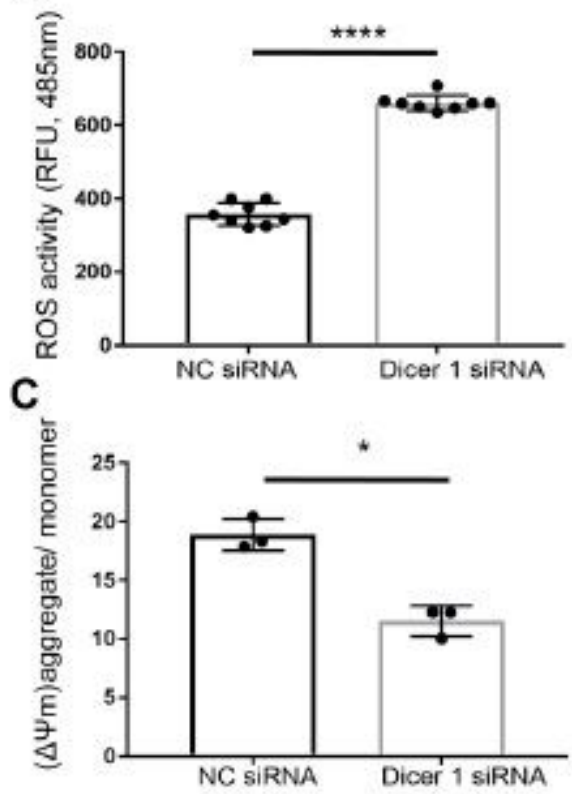

E

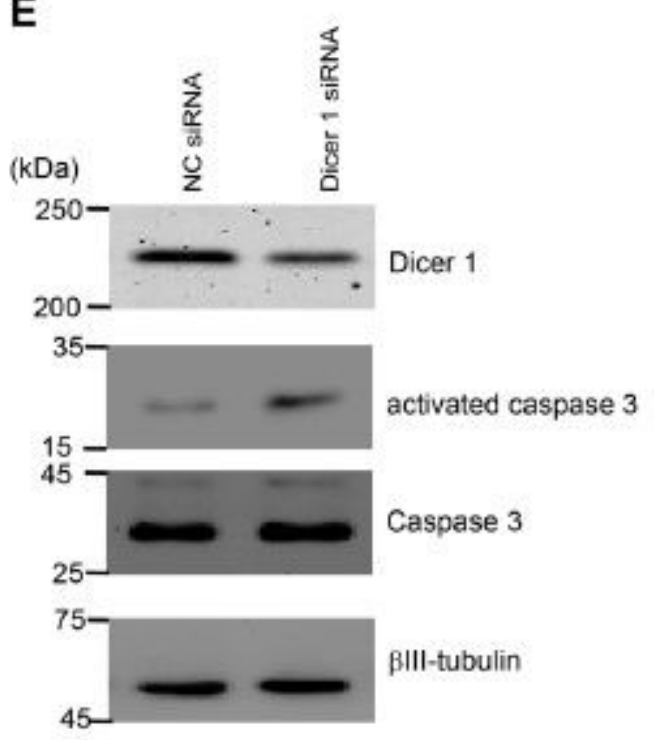

B
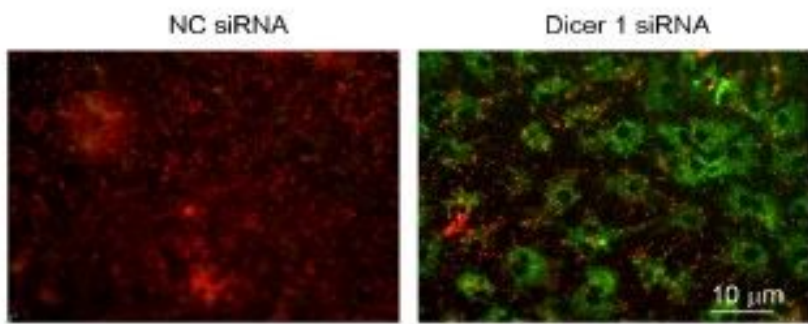

D

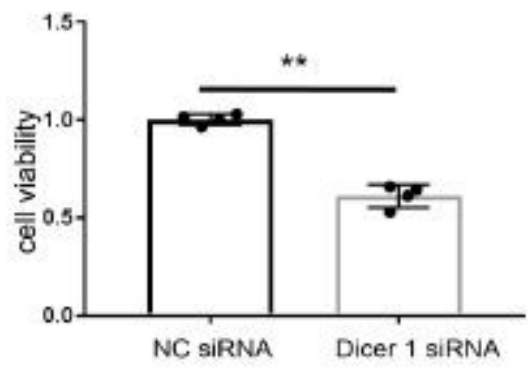

$\mathbf{F}$
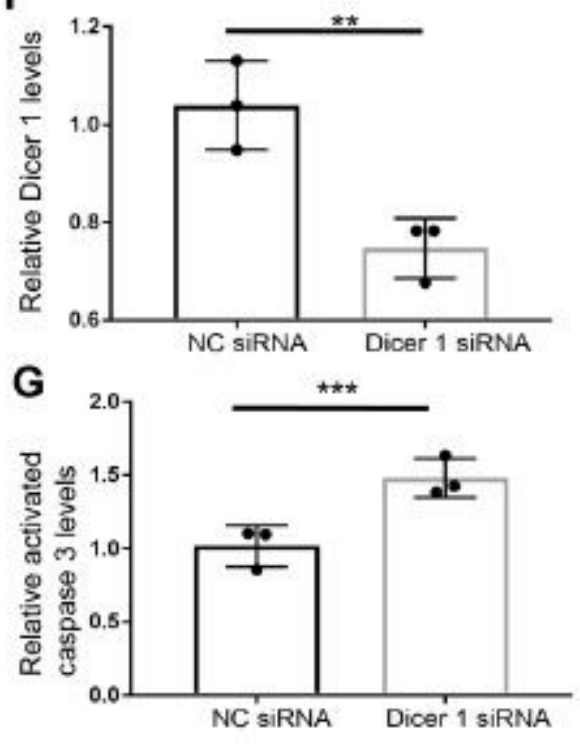

\section{Figure 2}

knocking down Dicer 1 induced production of reactive oxygen species (ROS), reduced mitochondrial membrane potential, enhanced apoptosis in primary murine hippocampal neuronal cultures. (A) The ROS levels in hippocampal neuronal cultures ( HNs) were measured by incubating 2',7'-

dichlorodihydrofluorescein diacetate for $20 \mathrm{~min}$ at $37 \mathrm{oC}$. The fluorescence values in relative fluorescence units (RFU) were acquired in a plate reader at $485 \mathrm{~nm}$ and were averaged from four independent culture preparation with duplicate cultures in each preparation. ${ }^{* \star \star *} p=0.00001$. (B) Representative images of JC1 staining inHNs subject to Dicer1 knockdown as above. Red fluorescence represented aggregate in mitochondria at hyperpolarized membrane potentials under healthy condition whereas green 
fluorescence as monomer in cytoplasm at depolarized membrane potentials under the context of compromised mitochondria. Bar $10 \mu \mathrm{m}$ for all panels. (C) Mitochondrial membrane potential $(\varangle \Psi \mathrm{m})$ of HNs subject to Dicer1 knockdown. The neurons were subject to Dicer1 knockdown and mitochondria (30 $\mu \mathrm{g}$ ) were isolated and stained with $\mathrm{JC}-1$. The values of mitochondrial membrane potential $(\nabla \Psi \mathrm{m})$ were indicated by the ratios between aggregated/monomer RFU and averaged from three independent culture preparations. Student's t-test was used to compare the differences, ${ }^{\star} p=0.0399$. (D) Viability of neurons subjected to Dicer1 knockdown. HNs were transfected with Dicer 1 siRNA or NC siRNA as described in the materials and methods. The absorption values at OD 450nm in Dicer1 siRNA group were measured and normalized to NC siRNA group. Student's t-test was used to compare the differences, ${ }^{* *} p=0.0012$ for comparison . (E) HNs were transfected with Dicer1 siRNA (50 pM) or control siRNA duplex (50 pM) per well and subjected to western blot examination of Dicer1, activated caspase3, caspase 3 and $\beta$ III-tubulin was used as a loading control. (F) The effects of knockdown by Dicer1 siRNA were normalized to those of NC siRNA and averaged from three independent culture preparation. Student's t-test was used to compare the differences, ${ }^{*} \mathrm{p}=0.044$ for comparison. (G) The optical densities of activated caspase- 3 relative to $\beta$ III-tubulin from $\mathrm{F}$ were normalized to NC siRNA group, and averaged from three independent culture preparation. Student's $t$-test was used to compare the differences, ${ }^{\star} p=0.02$ for comparison. 


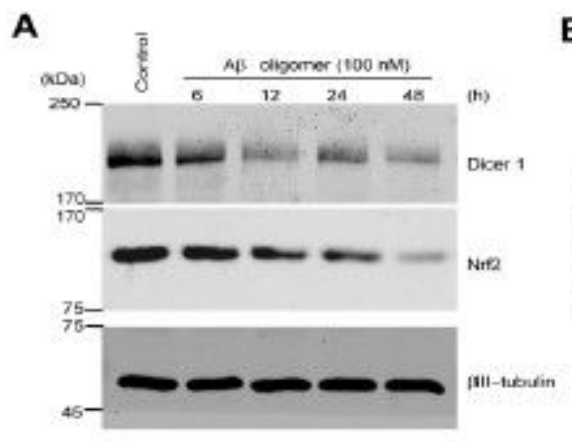

B $\quad$ C

D
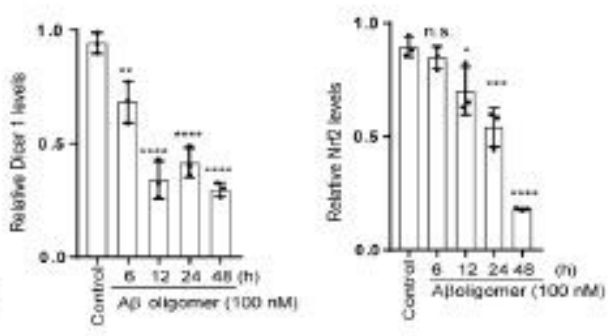

E

$\mathbf{F}$

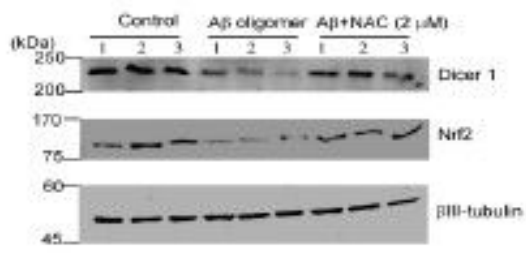

$\mathbf{G}$

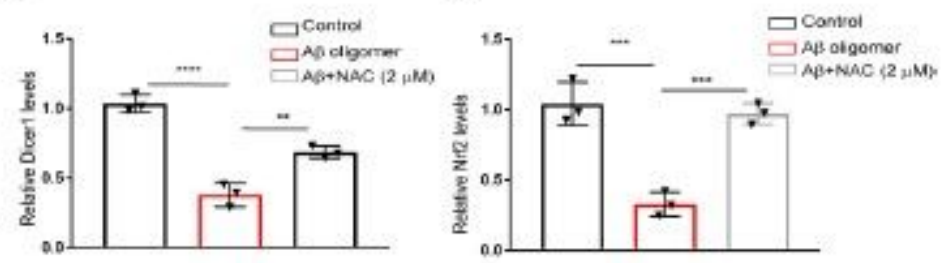

$\mathbf{H}$

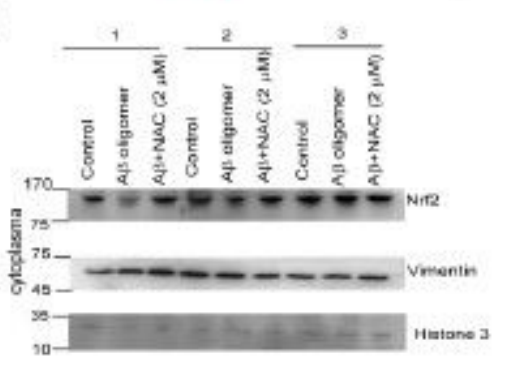

1
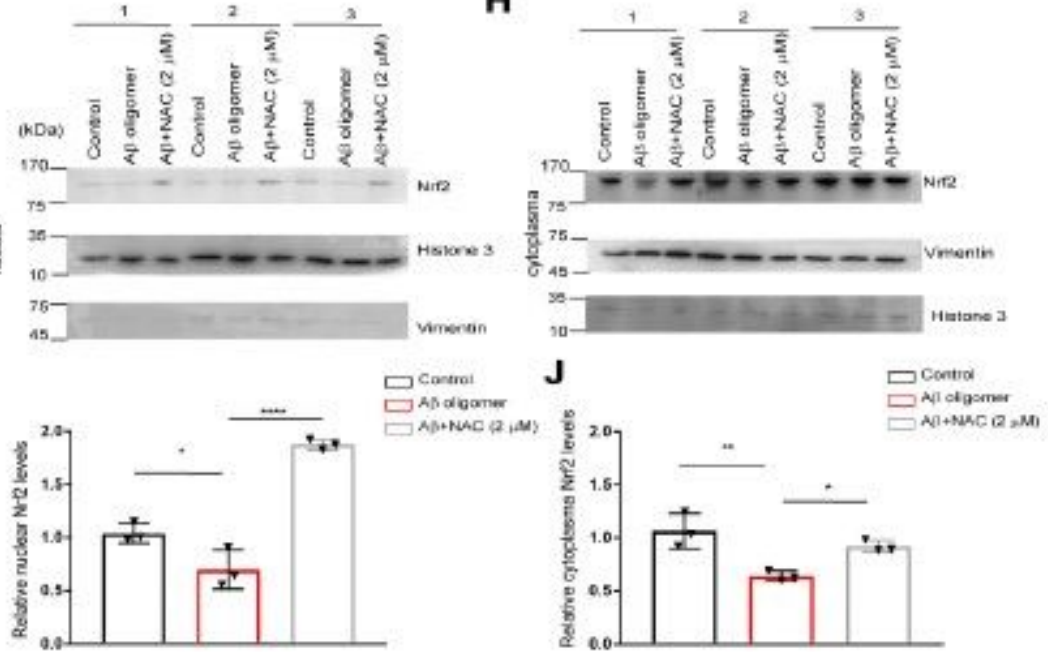

Figure 3

Chronic AB42 oligomer treatment reduced Dicer1 in neurons which was coupled with Nrf2 reduction, the effects rescued by NAC. (A-C) The time-course effect of $A \beta 42$ on Dicer1 or on Nrf2. The primary murine cortical neuronal cultures were subjected to vehicle or $A \beta$ oligomer treatment at indicated time. The cells were harvested for western blot for Dicer1 or Nrf2 and $\beta$ III-tubulin was used as a loading control. The relative optical densities of Dicer1 or Nrf2 from triplicate experiments were quantified and indicated in $B$ and $C .{ }^{* *} p<0.01,{ }^{* *} p<0.001,{ }^{* \star *} p<0.0001$, n.s indicated no significance. (D) The primary mouse cortical neurons were treated with $A \beta 42$ oligomer $(100 \mathrm{nM})$ and antioxidant NAC $(2 \mu \mathrm{M})$ for $48 \mathrm{~h}$. The neuronal cultures were harvested for western blot against Dicer 1, Nrf2, and Keap1. $\beta$ III-tubulin was used as an internal loading control. (E) The optical densities of Dicer1/ßlll-tubulin from D were normalized to sham treatment and averaged from three independent culture preparation. One-way ANOVA followed by Tukey's 
post hoc test was used to compare the differences. Control vs. $A \beta 42$ oligomer treatment, ${ }^{\star \star \star \star} p=0.00001$; $A \beta 42$ oligomer vs. $A \beta 42$ oligomer plus NAC treatment $(A \beta+N A C)$, ${ }^{*} p=0.032$. (F) The optical densities of $\mathrm{Nrf2/ \beta III-tubulin} \mathrm{from} D$ were normalized to sham treatment and averaged from three independent culture preparation. One-way ANOVA followed by Tukey's post hoc test was used to compare the differences. Control vs. $A \beta 42$ oligomer treatment, $* \star * p=0.0005 ; A \beta 42$ oligomer vs. $A \beta+N A C,{ }^{* \star *} p=0.0009$. (G) The neurons were treated with $A \beta 42$ oligomer $(100 \mathrm{nM})$ and antioxidant NAC $(2 \mu \mathrm{M})$ for $24 \mathrm{~h}$ and the nucleic proteins were extracted and blotted against Nrf2, and histone3 served as a loading control and vimentin as a negative control; $(\mathrm{H})$ the cytoplasmic proteins were isolated and blotted against $\mathrm{Nrf2}$, and vimentin served as a loading control and histone3 as a negative control. (I) The optical densities of nucleic Nrf2 relative to histone 3 averaged from three independent culture preparation in G. One-way ANOVA followed by Tukey's post hoc test was used to compare the differences. Control vs. $A \beta 42,{ }^{*} p=0.0326 ; A \beta 42$ vs. $A \beta+N A C,{ }^{* \star \star *} p=0.00001$. (J) The optical densities of cytosolic Nrf2/vimentin were normalized to sham treatment and averaged from three independent culture preparation in $\mathrm{H}$. One-way ANOVA followed by Tukey's post hoc test was used to compare the differences. Control vs. $A \beta 42$ oligomer, ${ }^{*} p=0.0076 ; A \beta 42$ oligomer vs. $A \beta+N A C,{ }^{*}=0.0419$.

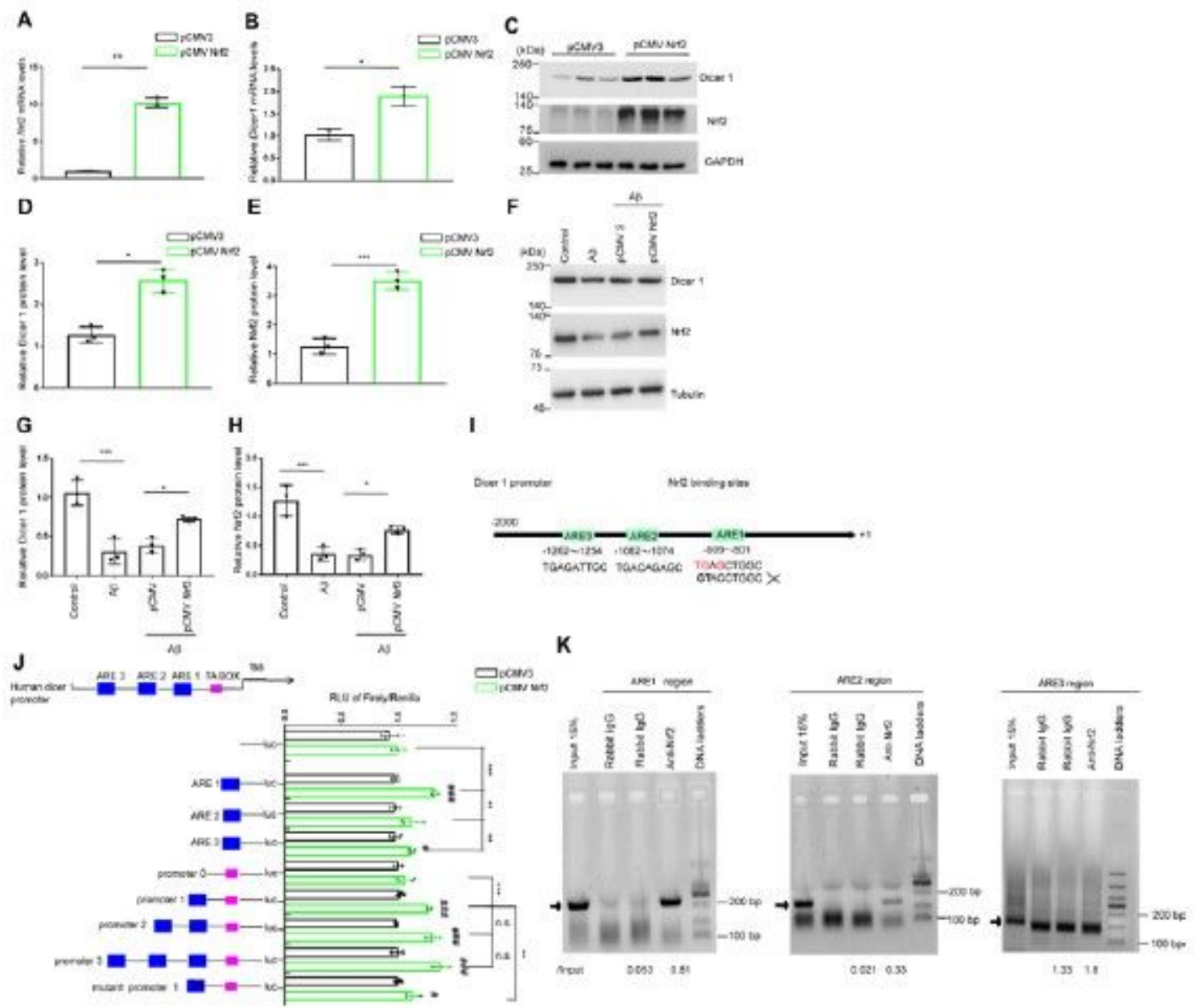

Figure 4 
Nrf2 regulated Dicer1 production via Nrf2-ARE pathway. Neuro-2a cells were transfected with mouse Nrf2expressing plasmids (pCMV Nrf2, 1.5 $\mu \mathrm{g}$ ) or equal amount of vehicle plasmid (pCMV3) in 6-well plates. Examination of Nrf2 or Dicer1 mRNA was performed by qRT-PCR analysis. (A-B) Determination of Nrf2 and Dicer 1 mRNA levels by overexpression of Nrf2 in Neuro-2A cells. Student's t-test was used to compare the differences. ${ }^{*} p=0.0016$ for comparison of the levels of Dicer1 mRNA, ${ }^{*} p=0.0482$ for comparison of the levels of Nrf2 mRNA. (C) Neuro-2a cells were transfected with a murine Nrf2expressing (pCMV Nrf2) or vehicle plasmid (pCMV3) by use of lipofectamine 2000 for $48 \mathrm{~h}$. At the end of the culture, the cells were harvested for western blot against Nrf2 and Dicer1. GAPDH was used as a loading control. (D) The protein levels of Dicer1/ GAPDH in C were normalized to pCMV3 group and averaged from three independent culture preparation. Student's t-test was used to compare the differences, pCMV3 vs. pCMV3 Nrf2, *p=0.0004. (E) The protein levels of Nrf2/GAPDH in C were normalized to $\mathrm{pCMV} 3$ group and averaged from three independent culture preparation. Student's t-test was used to compare the differences, $p C M V 3$ vs. pCMV3 Nrf2, ${ }^{\star \star *} p=0.024$. (F) Neuro-2a cells were subjected to treatment by vehicle, $A \beta 42$ oligomer $(100 \mathrm{nM})$ for $48 \mathrm{~h}$ and in parallel experiments, the cells were transfected with pCMV3 or pCMV3 Nrf2 for $48 \mathrm{~h}$ and then subjected to Aß42 for $48 \mathrm{~h}$. The cells were harvested for determination of Dicer1 and Nrf2 and Tubulin was used as a loading control. (G) The protein levels of Dicer1/ Tubulin in F were normalized to pCMV3 group and averaged from three independent culture preparation. Student's t-test was used to compare the differences, pCMV3 vs. pCMV3 Nrf2, ${ }^{*} \mathrm{p}=0.0004$. $(\mathrm{H})$ The protein levels of Nrf2/Tubulin in F were normalized to $\mathrm{pCMV3}$ group and averaged from three independent culture preparation. Student's t-test was used to compare the differences, $p C M V 3$ vs. pCMV3 Nrf2, ***p=0.024. (I) ARE motifs were found by use of the JASPAR database (http://jaspar.genereg.net/) within the Dicer1 promoter. (J) The upper panel showed scheme of Dicer 1 promoter containing three predicted ARE sites (blue square), TA box (pink red), tss indicating transcriptional start site (tss). HEK293 T cells were transfected with individual construct (150 ng), Renilla luciferase plasmid (7.5 ng), and PCMV3 or pCMV Nrf2 plasmid expressing human Nrf2 (150 ng). At the end of culture, the cells were harvested and subject to luciferase activity assay. The results were averaged from five independent culture preparation. One-way ANOVA followed by Tukey's post hoc test was used to compare the differences. Comparing co-transfection of pCMV3 Nrf2 with co-transfection of pCMV3 in individual construct, \#\#\# $\mathrm{p}=0.000219$ in ARE1, \#p=0.043 in ARE3, \#\#\#p=0.00047 in promoter 1 , $\# \# \# \mathrm{p}=0.000135$ in promoter $2, \# \# \mathrm{p}=0.000114$ in promoter 3 , and $\# \mathrm{p}=0.048$ in mutant promoter 1 . Under the condition of co-transfection of pCMV3 Nrf2, ARE1 vs. pGL6-luc, ${ }^{* \star} \mathrm{p}=0.00041$; ARE1 vs. ARE2, $\star \star \mathrm{p}=0.000825$; ARE1 vs. ARE3, $* \star \mathrm{p}=0.0093$; promoter 1 vs. promoter $0, * \star \star \mathrm{p}=0.000361$; promoter 1 vs. mutant promoter $1,{ }^{\star} \mathrm{p}=0.009$, promoter 1 vs. promoter $2, \mathrm{p}=0.194$ (n.s); promoter 1 vs. promoter 3 , $\mathrm{p}=0.254$ (n.s). (K) CHIP assay of the interaction between Nrf2 and the ARE motif-containing Dicer1 promoter in SK-N-BE cells. Arrows indicated the captured fragments containing ARE-1 or ARE-2 motif. The optical densities of bands were measured by Image $J$ and the ratios relative to positive input were shown below the individual bands, respectively. 

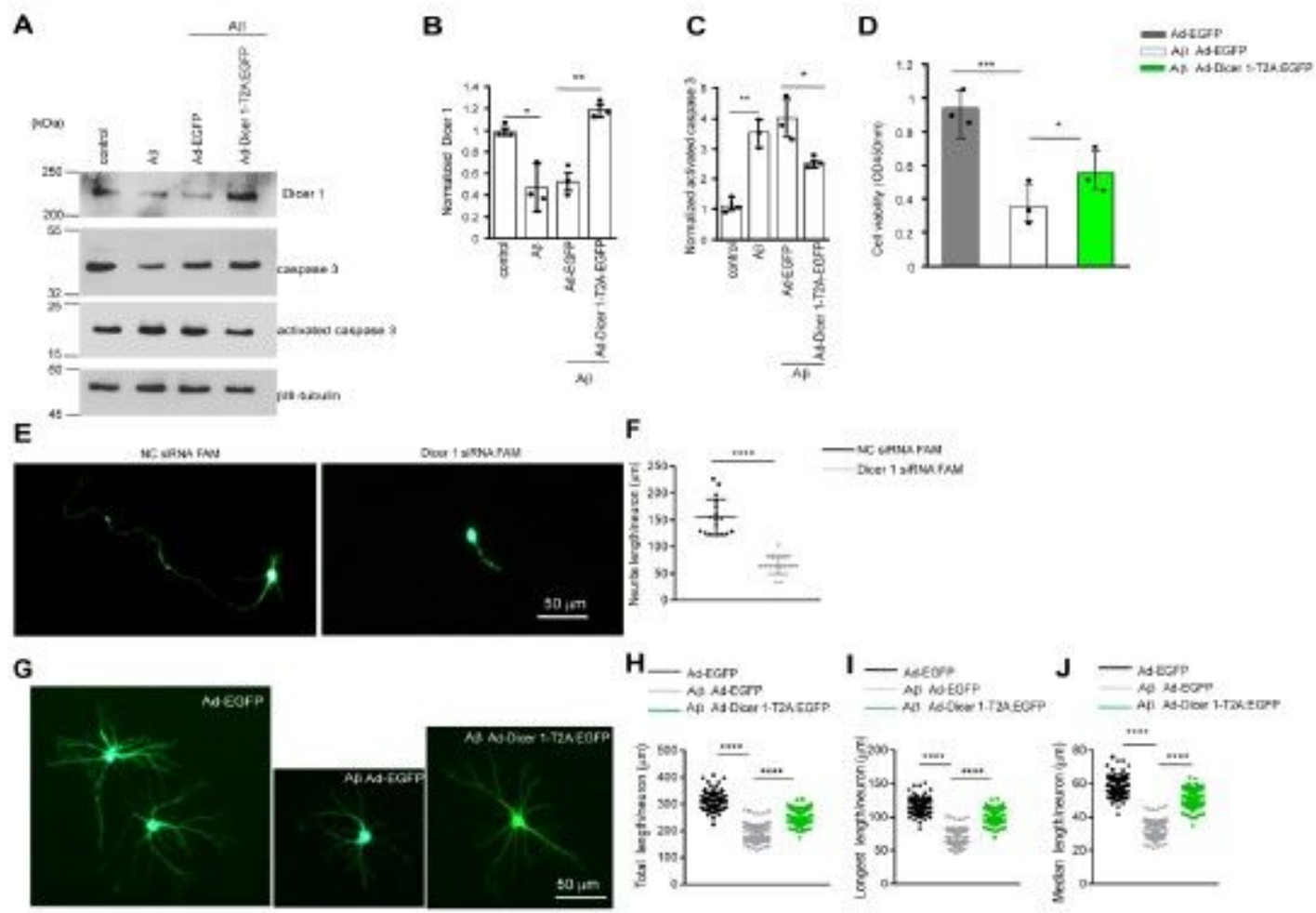

\section{Figure 5}

Overexpression of Dicer 1 reduced $A \beta 42$ oligomer-mediated apoptosis and neurite deficit in primary hippocampal neuronal cultures. (A) The primary murine hippocampal neurons at DIV 3 were treated with $\mathrm{A} \beta 42$ oligomer $(100 \mathrm{nM})$ for $24 \mathrm{~h}$ or subject to sham treatment in neurobasal medium plus $2 \% \mathrm{~B} 27$. In parallel experiments, the media were replaced with fresh neurobasal medium plus $2 \%$ B27 following Aß42 treatment, and then infected with vehicle virus, Ad-EGFP or Ad-dicer1-T2A:EGFP virus $(5 X 107 \mathrm{vg} / \mathrm{mL})$ for $48 \mathrm{~h}$. At the end of treatment, the cultures were harvested and homogenized for western blot against Dicer1, caspase 3, and activated caspase 3. $\beta$ III-tubulin was used as a loading control. (B) Dicer1 relative to $\beta$ III-tubulin were normalized to sham treatment and averaged from three independent culture preparation. One-way ANOVA followed by Tukey's post hoc test was used to compare the differences. Dicer 1 was reduced by $A \beta 42$ oligomer treatment compared to sham treatment ( ${ }^{*} p=0.033$ ) and the reduction of Dicer1 by $A \beta 42$ was rescued by infection of Ad-dicer1-T2A:EGFP compared to infection by vehicle virus ( ${ }^{\star *} p=0.0074$ ). (C) Activated caspase 3 relative to $\beta$ III-tubulin in A were normalized to sham treatment and averaged from three independent culture preparation. One-way ANOVA followed by Tukey's post hoc test was used to compare the differences. Activated caspase 3 was increased by A 42 oligomer compared to sham treatment ( $\left.{ }^{\star \star} p=0.0061\right)$, which was reduced by infection of Ad-dicer1-T2A:EGFP compared to infection by vehicle virus $\left({ }^{*} p=0.023\right)$. (D) The loss of cell viability of hippocampal neurons treated by $A \beta(* \star * p=0.00067)$ was significantly rescued by infection with Ad-dicer1-T2A:EGFP compared to infection by vehicle virus $\left({ }^{*} p=0.047\right)$. The values were averaged from quadruplicate cultures with three 
independent culture preparation. (E) Knockdown of Dicer1 reduced neurite length in primary cortical neuronal cultures. 7×104 cortical neurons were transfected with 6-fluorescein amidite (FAM)-labeling Dicer1 siRNA duplex (Dicer1 siRNA FAM) or scrambled siRNA duplex (NC siRNA FAM) for $12 \mathrm{~h}$, respectively. Neurons were observed with fluorescence microscope. The images were representative images of neurons transfected with Dicer1 siRNA FAM or NC siRNA FAM. Bar, $50 \mu \mathrm{m}$. (F) Total neurite length per cortical neuron averaged from 17 neurons under either transfection condition from E. Student's $t$-test was used to compare the difference, ${ }^{\star \star \star \star} p<0.0001$. (G) Representative hippocampal neurons (150 neurons for each type of treatment) infected by Ad-EGFP virus (Ad-EGFP), A 442 treatment followed by infection with Ad-EGFP virus ( $A \beta$ Ad-EGFP) and $A \beta 42$ treatment followed by infection with Ad-Dicer1T2A:EGFP virus (AB Ad-Dicer1:T2A:EGFP) were indicated. $(H)$ Total neurite length, $(I)$ the longest length and $(J)$ median length of hippocampal neurons with treatment from $\mathrm{G}$ were calculated and averaged. $A \beta 42$ treatment reduced neurite length compared to sham treatment which was rescued by infection with Ad-dicer 1-T2A:EGFP virus. ${ }^{\star \star \star \star} p<0.0001$, The neurite length averaged from 150 neurons in each treatment and One-way ANOVA followed by Tukey's post hoc test was used to compare the differences.

A

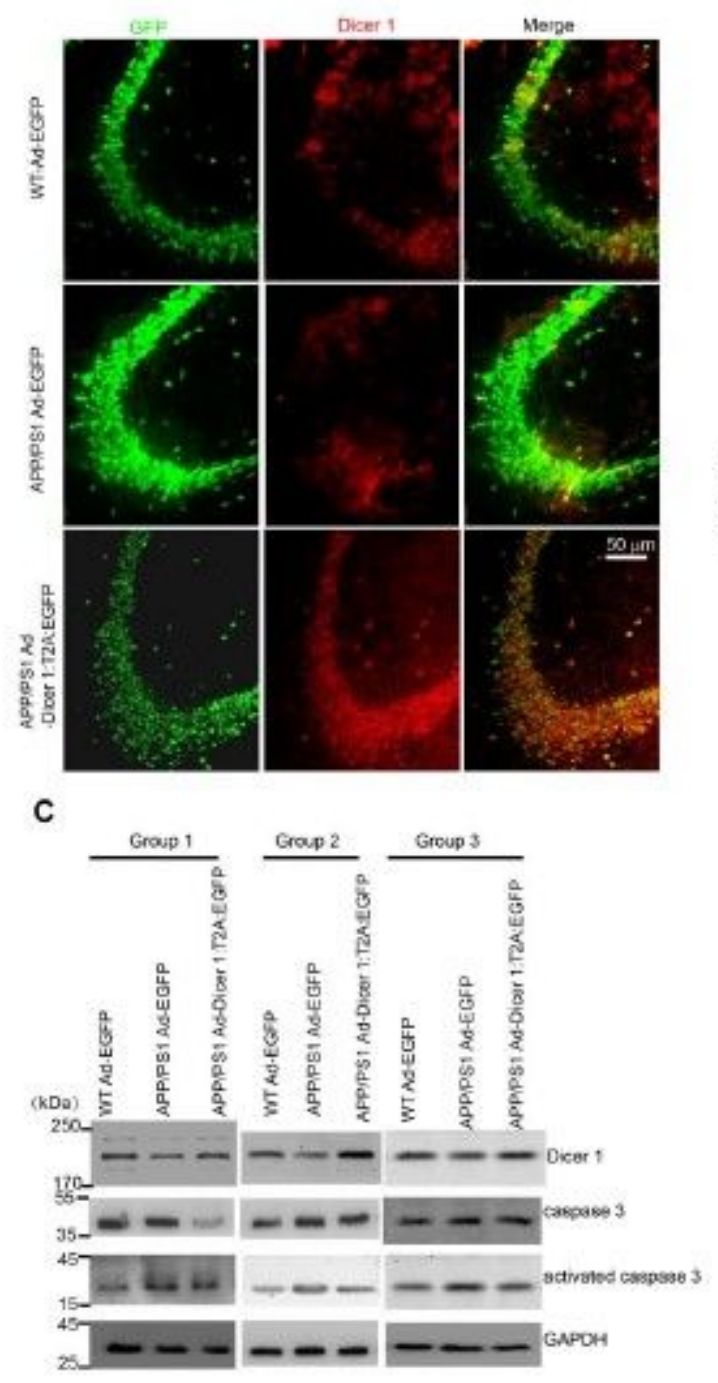

B
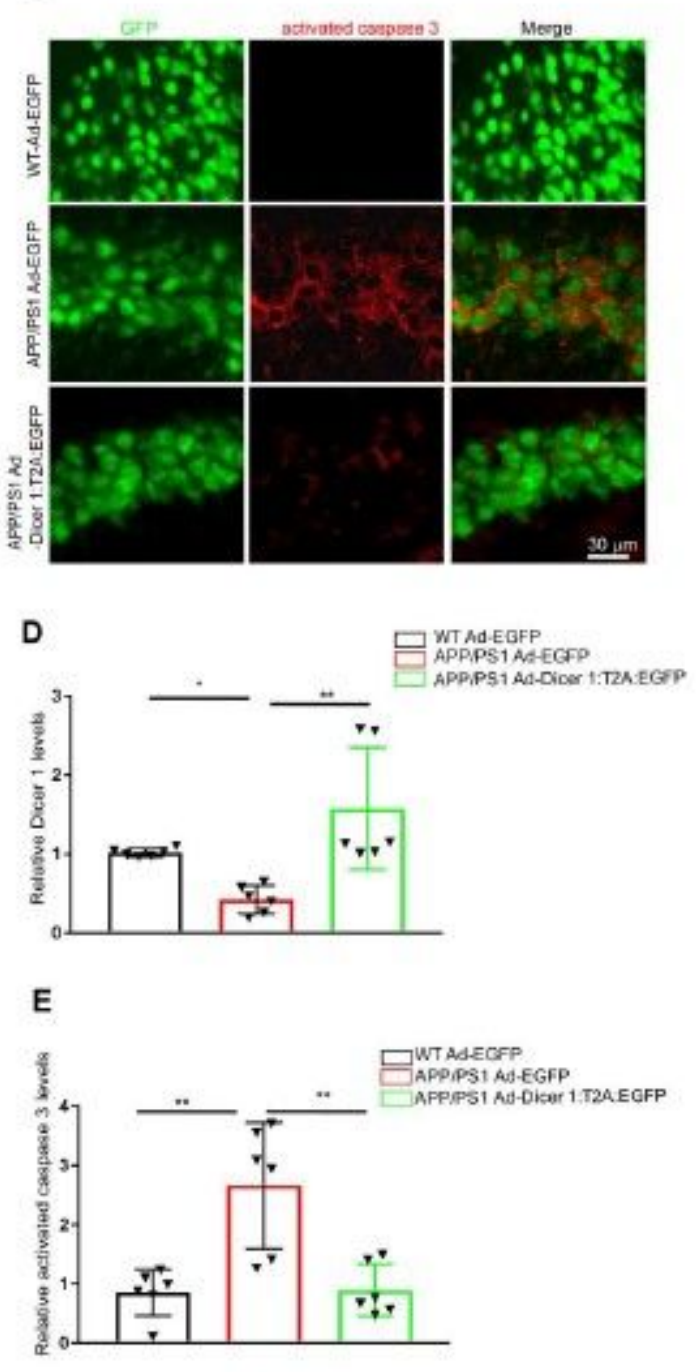


\section{Figure 6}

Overexpression of Dicer1 in APP/PS1hippocampus inhibited activated caspase 3. APP/PS1 mice or WT littermate at the age of 3.5-/4-month were injected with Ad-EGFP or Ad-Dicer1-T2A:EGFP virus (1.2 X109 $\mathrm{vg} / \mathrm{mL}$ ) in CA3 hippocampi. 17 days after injection, the injected mice were perfused and each brain was sagittally cut into $\sim 60$ sections at $10 \mu \mathrm{m}$-thick for ach section. The sections every other six sections were chosen for double staining of Dicer1 (red) (A) and activated caspase 3 (red) (B). The typical images of CA3 hippocampal region were indicated. (A) Dicer1 staining (red) in APP/PS1 CA3 hippocampus was reduced compared to those in WT littermate and infection with Ad-Dicer 1-T2A:EGFP virus dramatically increased Dicer1 staining in CA3 hippocampus. The images were typical of 60 sections from six mice. Bar, $50 \mu \mathrm{m}$. (B) The staining of activated caspase 3 (red) in APP/PS1mice was increased compared to those in WT and this increase in staining was mitigated by infection with Ad-Dicer1-T2A:EGFP virus. The images were typical of 60 sections from six mice. Bar, $30 \mu \mathrm{m}$. (C) The CA3 hippocampi were harvested, homogenized, and subjected to centrifugation at 12,000 X g, and the supernatants were used for western blot against Dicer1, caspase3, and activated caspase3, and typical blots were indicated. (D) Dicer1 was reduced in APP/PS1 hippocampi compared to WT ( $\left.{ }^{*} \mathrm{p}=0.0217\right)$, which was increased by infection with Ad-Dicer1-T2A:EGFP virus ( $\left.{ }^{\star \star} p=0.0093\right)$. The optical densities averaged from six mice in each group and Kruskal-Wallis test was used to compare the differences. (E) Activated caspase 3 relative to GAPDH was increased in APP/PS1 hippocampi compared to WT ( ${ }^{\star *} \mathrm{p}=0.00681$ ), which was reduced by infection with Ad-Dicer1-T2A:EGFP virus ( $\left.{ }^{\star \star} p=0.0067\right)$. The optical densities averaged from six mice in each group and Kruskal-Wallis test was used to compare the differences.

A

A

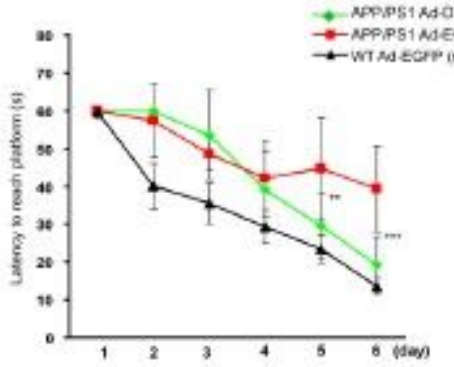

B B

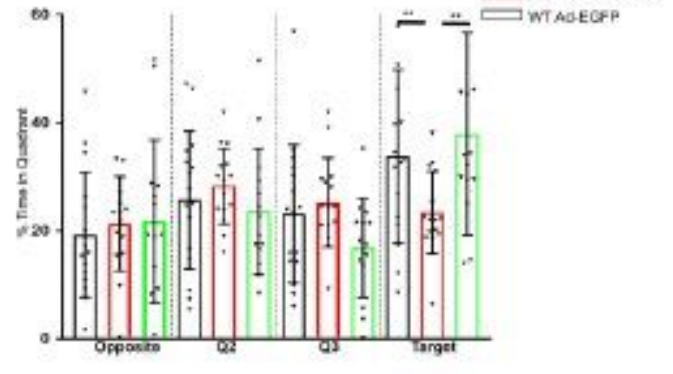

C

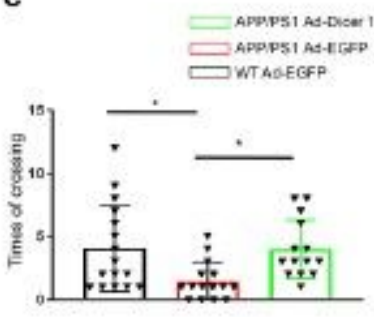

D

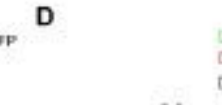

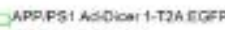
SAPP.PBS MSEGFP WNT AEECFP

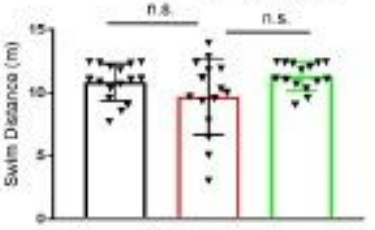

E
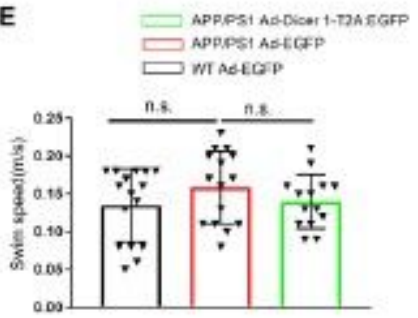

Figure 7 
Overexpression of Dicer 1 in CA3 hippocampus significantly improved spatial learning in APP/PS1 mice. Ad-Dicer1-T2A:EGFP virus $(1.2 \times 109 \mathrm{vg} / \mathrm{mL})$ or equal amount of Ad-EGFP virus were injected into CA3 hippocampus in APP/PS1 or WT mice, at the age of 3.5-4 months, and behavior test was conducted at 1723 days after injection. (A) Learning curve of the mice from WT mice injected with Ad-EGFP virus $(n=16)$, APP/PS1 mice injected with Ad-EGFP virus $(n=15)$ or Ad-Dicer1-T2A:EGFP virus $(n=14)$. The differences of latency to reach platform were analyzed by use of two-way ANOVA with repeated measures against day and treatment (virus injected), pday $=0.00001$, ptreatment $=0.0241$, pinteraction $=0.0148$. Tukey's post hoc test was further used to compare the differences between APP/PS1 mice injected with Ad-Dicer1T2A:EGFP virus and APP/PS1 mice injected with Ad-EGFP virus, ${ }^{\star \star} p=0.008$ on day 5 and ${ }^{* \star} \mathrm{p}=0.00001$ on day 6. (B) Analysis of probe trial indicated that preference of APP/PS1mice for the target quadrant was enhanced by Ad-Dicer 1-T2A:EGFP virus injection in CA3 hippocampi. Two-way ANOVA with repeated measures against quadrant and treatment was used to compare the differences, pquadrant $=0.1122$, ptype $=1$, pinteraction $=0.0046$. Tukey's post hoc test was further used to compare the differences among groups, APP/PS1 Ad-Dicer 1-T2A:EGFP $(n=16)$ vs. APP/PS1 Ad-EGFP( $n=15),{ }^{\star *} p=0.0087$; APP/PS1 Ad$\operatorname{EGFP}(n=15)$ vs. WT Ad-EGFP $(n=16),{ }^{*} p=0.0023$. (C) Frequencies of crossing target platform was increased by Dicer1 overexpression. Tukey's post hoc test was further used to compare the differences among groups, APP/PS1 Ad-Dicer 1-T2A:EGFP ( $n=14)$ vs. APP/PS1 Ad-EGFP $(n=15) ;{ }^{*}=0.043 ;$ APP/PS1 $\operatorname{Ad-EGFP~(~} n=15)$ vs. WT Ad-EGFP ( $n=16),{ }^{*} p=0.039$. (D) Swim distance and (E) Swim speed did not show differences among groups (n.s). 